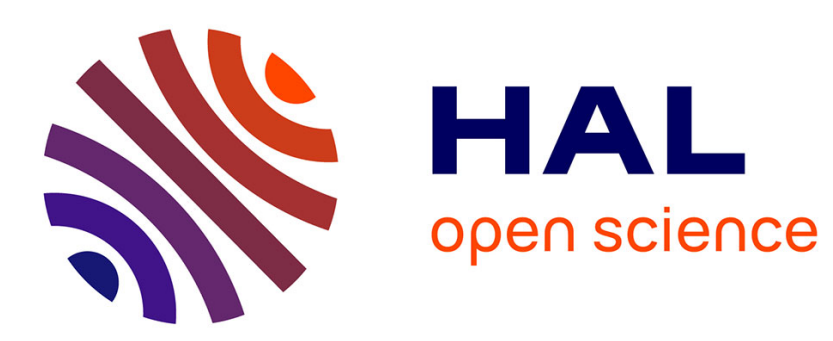

\title{
Palladium Nanoparticles in Polyols: Synthesis, Catalytic Couplings, and Hydrogenations
}

\author{
Isabelle Favier, Daniel Pla, Montserrat Gómez
}

\section{To cite this version:}

Isabelle Favier, Daniel Pla, Montserrat Gómez. Palladium Nanoparticles in Polyols: Synthesis, Catalytic Couplings, and Hydrogenations. Chemical Reviews, 2020, 120 (2), pp.1146-1183. 10.1021/acs.chemrev.9b00204 . hal-03011372

\section{HAL Id: hal-03011372 \\ https://hal.science/hal-03011372}

Submitted on 18 Nov 2020

HAL is a multi-disciplinary open access archive for the deposit and dissemination of scientific research documents, whether they are published or not. The documents may come from teaching and research institutions in France or abroad, or from public or private research centers.
L'archive ouverte pluridisciplinaire HAL, est destinée au dépôt et à la diffusion de documents scientifiques de niveau recherche, publiés ou non, émanant des établissements d'enseignement et de recherche français ou étrangers, des laboratoires publics ou privés. 


\title{
Palladium nanoparticles in polyols: synthesis,
}

\section{catalytic couplings and hydrogenations}

\author{
Isabelle Favier,,$^{\dagger}$ Daniel Pla, ${ }^{*},+$ and Montserrat Gómez ${ }^{*},+$ \\ † Laboratoire Hétérochimie Fondamentale et Appliquée (UMR 5069), Université de Toulouse, \\ CNRS, 118 route de Narbonne, 31062 Toulouse Cedex 9, France.
}

ABSTRACT: Alcohols, in particular polyols, are well-known for the synthesis of metal nanoparticles often acting as reducing agents, solvents, and stabilizers. Given not only their structural flexibility depending on the number of $\mathrm{OH}$ functions and their inherent $\mathrm{H}$ bonding interactions, but also the wide range of polyol molecular weights readily available, different physico-chemical properties (boiling point, polarity, viscosity) could be exploited towards the synthesis of well-defined nanomaterials. In particular, the relevance of the supramolecular structure of polyols has a fundamental impact on the formation of metal nanoparticles thereby favoring the dispersion of the nanoclusters. In the field of the metal-based nanocatalysis, palladium occupies a privileged position mainly due to its remarkable versatility in terms of reactivity representing a foremost tool in synthesis. In this review, we describe the controlled synthesis of Pd-based nanoparticles in polyol medium focusing on the progress in terms of tailoring size, morphology, structure, and surface state. Moreover, we discuss the use of 
palladium nanoparticles, in a polyol solvent, applied in two of the most relevant Pd-catalyzed processes, i.e. couplings and hydrogenation reactions, including multi-step processes.

\section{CONTENTS}

1. Introduction

2. Synthesis of palladium-based nanoparticles in polyol medium

2.1. Palladium monometallic nanoparticles (PdNPs)

2.1.1. Ethylene glycol and its diol derivatives

2.1.2. Glycerol and other polyols

2.2. Palladium bimetallic nanoparticles

2.2.1. Palladium bimetallic nanoparticles containing $4 \mathrm{~d}$ and $5 \mathrm{~d}$ transition metals

2.2.2. Palladium bimetallic nanoparticles containing $3 \mathrm{~d}$ transition metals

2.3. Hydroxyl compounds from natural sources

3. Pd-based nanocatalysts applied in hydrogenations

3.1. Hydrogenation reactions catalyzed by PdNPS in PEG

3.2. Hydrogenation reactions catalyzed by PdNPS in glycerol

4. Pd-based nanoparticle leveraged coupling reactions

4.1. Dimerization of alkynes 
4.2. $\mathrm{C}(\mathrm{sp})-\mathrm{C}\left(\mathrm{sp}^{2}\right)$ Sonogashira cross-coupling

4.3. $\mathrm{C}\left(\mathrm{sp}^{2}\right)-\mathrm{C}\left(\mathrm{sp}^{2}\right)$ Hiyama-Denmark cross-coupling

4.4. $\mathrm{C}\left(\mathrm{sp}^{2}\right)-\mathrm{C}\left(\mathrm{sp}^{2}\right)$ Heck-Mizoroki cross-coupling

4.5. $\mathrm{C}\left(\mathrm{sp}^{2}\right)-\mathrm{C}\left(\mathrm{sp}^{2} / \mathrm{sp}^{3}\right)$ Suzuki-Miyaura cross-coupling

4.5.1. In PEG and glycerol media

4.5.2. Solid-supported nanocatalysts

4.6. $\mathrm{C}\left(\mathrm{sp}^{2}\right)-\mathrm{C}\left(\mathrm{sp}^{2}\right)-\mathrm{C}\left(\mathrm{sp}^{2}\right)$ Carbonylative Suzuki cross-coupling

4.7. $\mathrm{C}\left(\mathrm{sp}^{2}\right)-\mathrm{C}\left(\mathrm{sp}^{2}\right)$ Ullmann-type homo-coupling

4.8. Miscellaneous: Si-N and C-N amination reactions

5. Conclusions and outlook

Author information

Corresponding authors

ORCID

Notes

Biographies

Acknowledgments

Abbreviations 
References 


\section{INTRODUCTION}

Certainly, palladium occupies a prominent place in catalysis for its efficiency in downstream wide-ranging processes $(\mathrm{C}-\mathrm{C}$ and $\mathrm{C}$-heteroatom bond formation reactions, hydrogenations, hydrodehalogenations, carbonylations, oxidations). ${ }^{1,2}$ Specially, the relevance of palladium in catalysis has been recognized in 2010 by the Nobel Prize in Chemistry awarded to Richard F. Heck, Ei-ichi Negishi, and Akira Suzuki for their original contributions in Pd-catalyzed crosscoupling reactions applied in organic synthesis of interest for both fundamental and applied purposes. $^{3}$ The ascertained versatility of palladium can be attributed to its ability to stabilize a large variety of matter states at different oxidations states, by appropriate tuning of conditions: organometallic complexes, nanoparticles, and extended surfaces, including polymetallic materials; furthermore, these different Pd-based species have proven their compatibility with different types of solids, permitting their immobilization and in consequence their recycling,

aspect of foremost importance in the frame of developing sustainable industrial processes. ${ }^{4-6}$ Concerning palladium nanoparticles, Bönnemann ${ }^{7}$ and Reetz ${ }^{8}$ independently described the first syntheses of palladium nanocatalysts in conventional organic solvents prepared by bottom-up (palladium salt chemical reduction) and top-down (electrochemical process transforming palladium bulk into palladium nanoparticles), respectively, for applications in $\mathrm{C}$-C crosscoupling reactions.

Focusing on nano-scale materials, metal nanoparticles (well-defined entities with dimensions in the range $1-100 \mathrm{~nm}$ ) have experienced a huge development over the last four decades due to their unique physical and chemical properties in comparison to materials belonging to higher or lower scales. This is in fact mainly thanks to the electronic and structural effects of nanomaterials, thus enabling a large panel of catalytic applications. ${ }^{9-13}$ Although, metal 
nanoparticles have been applied for a long time in heterogeneous catalysis, contributing to important industrial developments mainly associated to oil sector, ${ }^{14}$ nanocatalysis has emerged at the end of 20 century, combining colloidal nanoparticles dispersed in a solvent and nano-entity engineering granting a control of size, shape, and surface state of the as-prepared objects. The advances towards the characterization and tailoring of nano-objects enable catalyst tuning and design at the nanometric scale. ${ }^{15-18}$ In this frame, reproducible synthetic methodologies able to produce metal nanoparticles with a tight control of structure, morphology, composition, and surface state are crucial for their applications. The synthetic approaches towards metal nanoparticles are generally classified as physical (top-down) and chemical (bottom-up) methods. ${ }^{19,20}$ The latter ones involve different types of solvents, from conventional (generally coming from fossil sources) to alternative ones (showing a lower environmental impact), such as water, ${ }^{21,22}$ ionic liquids, ${ }^{23} \mathrm{scCO}_{2},{ }^{24-26}$ polyols. ${ }^{27-29}$ Usually, these greener solvents, in contrast to volatile organic compounds, can play several roles, such as stabilizer, reductant and/or liquid phase for trapping nanocatalysts thus facilitating their recycling. This is in particular the case of polyols. In 1989, thirty years ago, the polyol methodology was for the first time applied by Fiévet and co-workers with the aim of reducing metal salts in liquid polyols (mainly using ethylene glycol), to lead to the formation of well-defined metal-based nanoparticles; ${ }^{30,31}$ thus, the polyol acts as a polyfunctional component, i.e. solvent of the metal precursors (relative high dielectric constant), reducing agent (relative low reduction potentials) and in some cases stabilizer (mostly for higher polyols, such as polysaccharides, polyphenols... see section 2.3), preventing the agglomeration of the generated metal colloids. ${ }^{27,32}$ In addition, due to their polarity and high boiling points, polyols are suitable solvents to be applied in microwave-assisted synthesis of metal nanoparticles. ${ }^{33-35}$ 
The present review aims at describing both the synthesis of palladium nanoparticles in polyol medium, as well as their use as nanocatalysts in leveraging cross-couplings and hydrogenations, including polyol-modified supports for catalytic applications. The catalyst recycling is a crucial aspect both from an environmental point of view and industrial application purposes. Catalyst recyclability assessment requires studies leading to consistent high conversions and/or yields (no more than $1-2 \%$ decrease in at least $5-9$ runs), or minimum total turnover numbers $c a .100,000{ }^{6}$ Obviously, the recycling capability of a given catalyst is intimately related to the nature of the catalytically active species. Thus, kinetic studies, deactivation data, and catalyst characterization after catalytic reactions are necessary to conclude about the catalyst recyclability. ${ }^{36-38}$ Taking into account this definition of catalyst recyclability, only those Pd-based systems meeting the aforementioned criteria will be considered as recyclable in the frame of this review.

Notably, this review pays special attention to characterization aspects and mechanistic insights. This contribution includes the works published until the end of February 2019. Electrocatalytic approaches are beyond the scope of this contribution.

\section{Synthesis of palladium-based nanoparticles in polyol medium}

In this part, we mainly focus on the contributions related to understanding the controlled synthesis of Pd-based NPs by the polyol approach, due to the impact that size and morphology produce on their properties and consequently in their applications; this discussion is presented by type of polyol, considering both mono and bimetallic Pd-based nanoparticles. This section focuses on the discussion of the synthesis of Pd-based nanoparticles by bottom-up methodologies under homogeneous conditions. Thus, the synthesis of supported nanoparticles has not been taken into consideration. 


\subsection{Palladium monometallic nanoparticles (PdNPs)}

Ethylene glycol (EG) has been typically used in the polyol synthesis of metal nanoparticles. ${ }^{32}$ In the case of palladium, PdNPs often exhibit single-crystal structures (cubes, bars, cuboctahedra), mainly due to the fast reduction in EG at relative high temperature. However, tuning the reaction conditions and/or using other polyols, the reaction kinetics evidently changes influencing both the type of seeds and their growth rate. In general, the shape of the NPs is mainly determined by growth rates of different nuclei facets. ${ }^{39}$ It is important to highlight that polyols are predominantly not appropriate stabilizers, requiring the presence of capping agents in order to avoid the agglomeration of the nano-objects during their preparation. However, Arora and coworkers have prepared quasi-spherical PdNPs $(c a .6 \mathrm{~nm})$ using palladium dichloride as precursor and EG acting as solvent, stabilizer and reducing agent; this approach has permitted to evidence the presence of monoalkoxides at the surface of isolated PdNPs by FTIR, TGA and AFM analyses. $^{40}$

In this section, we will particularly analyze the contributions exhibiting a control on the structure on the synthesized PdNPs. We firstly discuss the works related to EG and its diol derivatives [diethylene glycol (DEG), triethylene glycol (TrEG) and tetraethylene glycol (TEG)], followed by glycerol and other polyols.

\subsubsection{Ethylene glycol and its diol derivatives}

Reproducible methodologies leading to well-defined shape of nanoparticles is crucial for controlling their properties. In 2005, Xia and co-workers reported the first elucidation concerning the synthesis of uniform cuboctahedral PdNPs by a polyol methodology. ${ }^{41}$ They monitored the formation of PdNPs in EG at $110{ }^{\circ} \mathrm{C}$ in the presence of PVP using $\mathrm{Na}_{2}\left[\mathrm{PdCl}_{4}\right]$ as metal 
precursor; at $5 \mathrm{~min}$, the reaction mixture was mainly constituted by cuboctahedra together with twinned particles ( $c a .10 \%)$. The latter dissolved after $1 \mathrm{~h}$ reaction and the cuboctahedral PdNPs grew to $8 \mathrm{~nm}$, reaching $10 \mathrm{~nm}$ after $c a .8 \mathrm{~h}$ of reaction, as evidenced by HRTEM analysis (Figure 1). Both chloride anions and $\mathrm{O}_{2}$ were responsible for the oxidative etching of twinned NPs (under Ar, the twinned NPs remained stable over time) that only disappeared by exposing the sample to air. Thus, twinned NPs are more reactive due to the higher concentration of surface defects than cuboctahedra; in consequence, the presence of an oxidant in reaction medium provides a way to control the formation of PdNPs exhibiting a well-defined morphology.
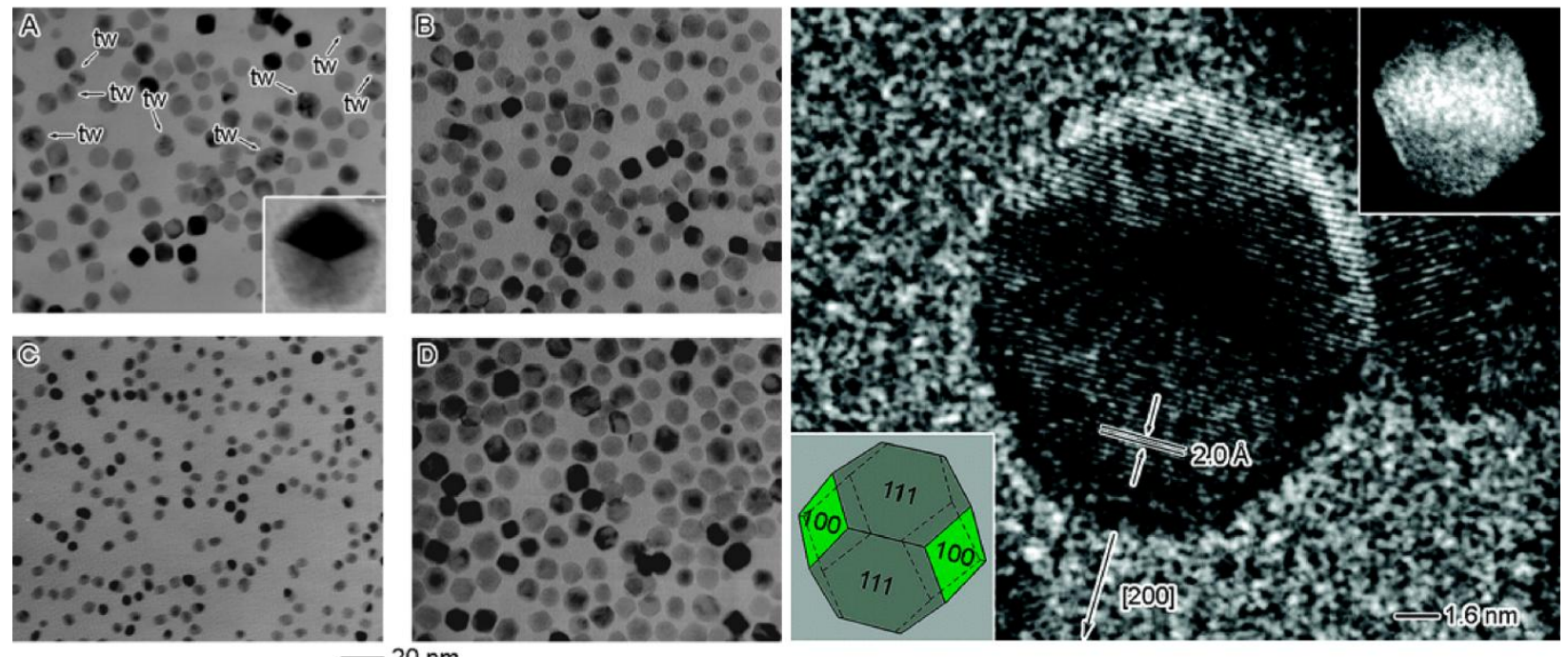

Figure 1. Left: TEM images prepared in EG at $110{ }^{\circ} \mathrm{C}$ in air at different times: $5 \mathrm{~min}(\mathrm{~A}) ; 3 \mathrm{~h}$ (B); $5 \mathrm{~h}(\mathrm{C}) ; 7 \mathrm{~h} 40 \mathrm{~min}(\mathrm{D})$; the inset of (A) shows the magnified image of a 5 -fold twinned nanoparticle (tw denotes twinned particles). Right: HRTEM image of a Pd cuboctahedron prepared at $3 \mathrm{~h}$ of reaction; insets show the dark-field TEM image of a cuboctahedron using the $\{200\}$ reflection beam (upper right) and the geometrical model of the cubooctahedron (lower left). Reprinted with permission from reference ${ }^{41}$. Copyright (2005) American Chemical Society. 
It is known that icosahedral structure is favored over both decahedral and cuboctahedral arrangements for small PdNPs [i.e. for total metal atoms less than 309]. ${ }^{42}$ In EG, PdNPs showing icosahedral structure (mean sizes from 5 to $35 \mathrm{~nm}$ ) were prepared by adding $\mathrm{HCl}$ to slow the reaction rate. ${ }^{43}$ Using DEG at $105{ }^{\circ} \mathrm{C}$ for the reduction of $\mathrm{Na}_{2}\left[\mathrm{PdCl}_{4}\right]$ in the presence of PVP, decahedral or icosahedral PdNPs were mainly obtained by adjusting the reaction medium through the presence of additives. ${ }^{44}$ The increase of acidity of the reaction medium by addition of $\mathrm{HCl}$ hampered the reduction of $\left[\mathrm{PdCl}_{4}\right]^{2-}$ anionic complex in both EG and DEG. In contrast, the addition of $\mathrm{Na}_{2} \mathrm{SO}_{4}$ triggers an increase of reducing properties of DEG due to hydrogen bonds formed between $\mathrm{SO}_{4}{ }^{2-}$ and the hydroxyl groups of DEG, favoring the oxidation of the polyol to the corresponding aldehyde, and then improving their reducing properties.

The same group has quantitatively studied the correlation between reduction rates of the metal precursor and structure for different diols (EG, DEG and TrEG). ${ }^{45}$ Thus, combining the data obtained at different temperatures (ranging from 60 to $140{ }^{\circ} \mathrm{C}$ ) for the three polyols, they concluded that for low initial reaction rate $\left(c a \cdot 10^{-8} \mathrm{Ms}^{-1}\right)$, a stacking-fault-lined structure was privileged, while at high rates ( $c a$. higher than $10^{-4} \mathrm{Ms}^{-1}$ ), single-crystals were mainly formed; twinned icosahedra were selectively formed in the narrow range of $8.7 \times 10^{-6}$ to $4.0 \times 10^{-7} \mathrm{Ms}^{-1}$ (Figure 2). Reaction times for truncated octahedra (or icosahedra), and nanoplates syntheses were $3 \mathrm{~h}$ and $30 \mathrm{~h}$, respectively. ${ }^{45}$ However, the authors did not report under which atmosphere the reactions were conducted. 


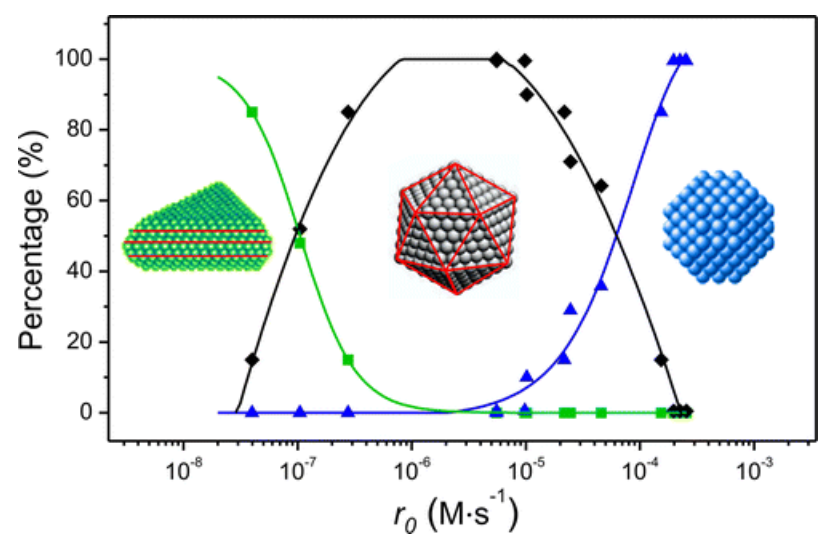

Figure 2. Schematic plot indicating the relative distribution of the twin structure of $\mathrm{Pd}$ nanocrystals as a function of the initial reaction rate $\left(\mathrm{r}_{0}\right)$ of a synthesis. Percentages of singlecrystal, multiply twinned, and staking fault-lined structures are denoted in blue, black, and green colors, respectively, and determined by TEM analyses. The twin planes or stacking faults are in red. Reprinted with permission from reference. ${ }^{45}$ Copyright (2015) American Chemical Society.

Teranishi and co-workers, following a similar protocol described by Xia in EG, ${ }^{41}$ but under slow reaction conditions, could control the selective formation of icosahedral PdNPs. ${ }^{46}$ They studied the influence of the reaction temperature, ranging from $100{ }^{\circ} \mathrm{C}$ to $150{ }^{\circ} \mathrm{C}$ in air. At high temperature $\left(150^{\circ} \mathrm{C}\right)$, small (mean size in the range 2-6 $\mathrm{nm}$ ) and spherical NPs were obtained; however, from $140{ }^{\circ} \mathrm{C}$ to $100{ }^{\circ} \mathrm{C}$ only icosahedra were formed with sizes from 15 to $42 \mathrm{~nm}$ (Figure 3). The as-prepared icosahedral NPs were stable in air for months. By PXRD, they could prove the high density of twin boundaries and sharp edges on the surface of icosahedral PdNPs. Under harsher conditions $\left(160^{\circ} \mathrm{C}\right)$ and in the presence of small amounts of silver nitrate, $\mathrm{Na}_{2}\left[\mathrm{PdCl}_{4}\right]$ was reduced in EG with PVP to form PdNPs of $c a .10 \mathrm{~nm}$ of mean diameter, showing different shapes. ${ }^{47,48}$ Despite the authors do not explicitly comment on the reaction atmosphere used to carry out the syntheses, their protocols seem to be in air. ${ }^{47,48}$ At higher 
temperature or longer times, only (quasi)spherical PdNPs were observed from $\mathrm{H}_{2}\left[\mathrm{PdCl}_{4}\right]$ or $\mathrm{K}_{2}\left[\mathrm{PdCl}_{4}\right]$ as metal precursor in air. ${ }^{49-51}$
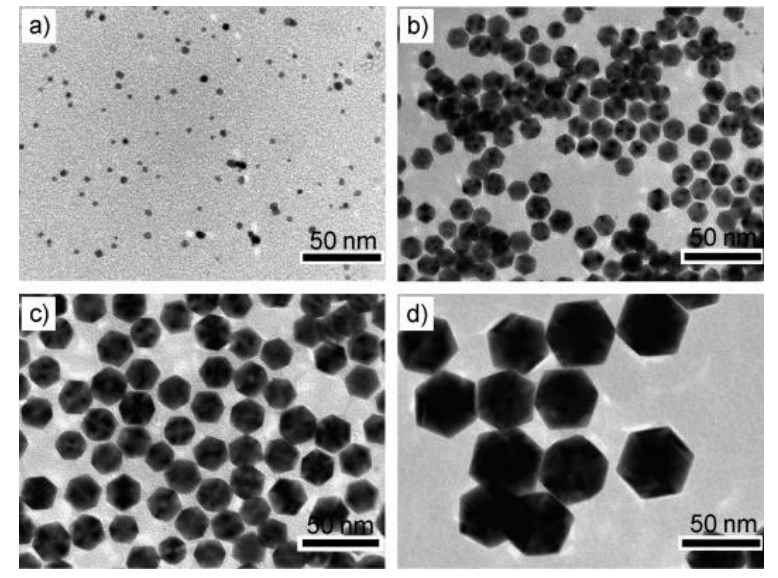

Figure 3. TEM micrographs of PdNPs synthesized at different temperatures: a) $150{ }^{\circ} \mathrm{C}$, b) 140 ${ }^{\circ} \mathrm{C}$, c) $130{ }^{\circ} \mathrm{C}$, and d) $100{ }^{\circ} \mathrm{C}$. Reproduced with permission from Ref. 46. Copyright (C) 2009 WILEY - VCH Verlag GmbH \& Co. KGaA, Weinheim.

For noble metals, microwave-assisted heating can induce faster nucleation processes than conventional activation, leading to reproducible synthesis and controlled structures in air. ${ }^{52}$ Notably, the authors report that the working temperature of $150^{\circ} \mathrm{C}$ could be quickly achieved by preheating the samples below the temperature of nucleation $\left(76^{\circ} \mathrm{C}\right)$ before applying a high power pulse to reach the desired temperature for controlled nucleation within seconds. ${ }^{52}$ In particular, for Pd, small cuboctahedra could be exclusively obtained in contrast to conventional heating, resulting in a range of sizes and morphologies. Moreover, under microwave irradiation, a mixture of nanocubes and nanorods was observed in the synthesis of PdNPs from $\mathrm{H}_{2}\left[\mathrm{PdCl}_{4}\right]$ in TEG in the presence of PVP and CTAB as surfactant. ${ }^{53}$ Despite the authors do not report if the synthesis was performed under a controlled atmosphere or in air using a modified domestic microwave oven operating at $900 \mathrm{~W}$, the in situ formed $\left[\mathrm{PdBr}_{4}\right]^{2-}$ anions (chloride exchange by 
bromide present in $\mathrm{CTAB}$ ) could be slowly reduced; it is important to note that under similar conditions but using EG instead of TEG, only spherical NPs were obtained. This behavior can be explained because TEG is a milder reducing agent showing higher viscosity in comparison with EG; these conditions can slow down both the nucleation and growth rate of PdNPs favoring the formation of large nanocubes ( $c a .24 \mathrm{~nm}$ ) with the presence of nanorods. Following a similar methodology, Gao and co-workers managed to synthesize selectively nanorods in EG; SQUID measurements evidenced the ferromagnetic properties of the as-prepared Pd nanorods, at room and lower temperatures (reaction atmosphere not described in the synthetic protocol). ${ }^{54}$ As discussed above, different polygonal PdNPs structures can be formed in $\mathrm{EG}$ at $85^{\circ} \mathrm{C}$ in air, using PVP as stabilizer, due to its selective adsorption on $\{111\}$ and $\{100\}$ facets. ${ }^{55}$ With the aim of obtaining anisotropic structures, a mixture of two surfactants, PVP and CTAB, was necessary, by optimization of the appropriate $\mathrm{PVP} / \mathrm{CTAB}$ ratio and the addition of $\mathrm{Fe}(\mathrm{III})$ salts in acid medium (Figure 4).
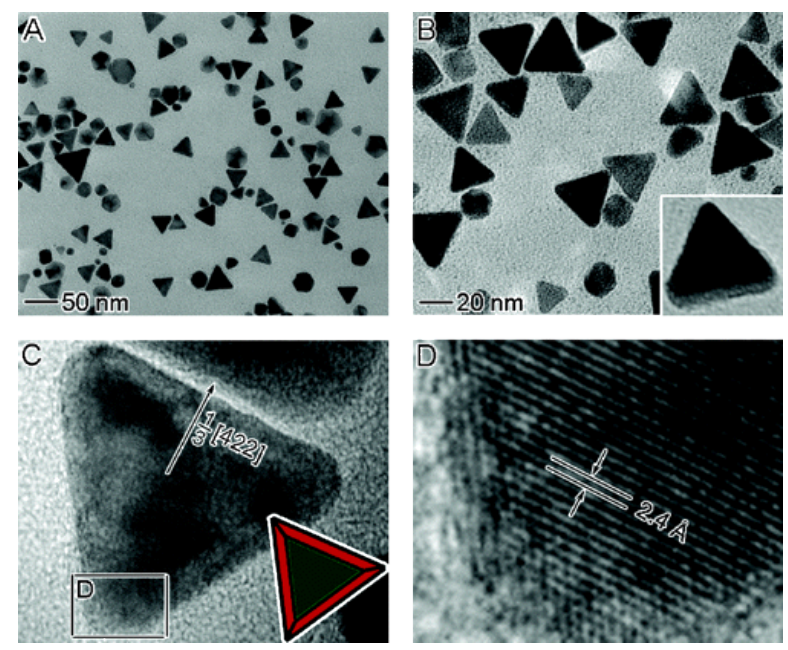

Figure 4. Electron microscopy characterization of Pd triangular nanoplates prepared at $85{ }^{\circ} \mathrm{C}$ in the presence of $0.36 \mathrm{mM} \mathrm{FeCl}_{3}$ and $5 \mathrm{mM} \mathrm{HCl}$, with the molar ratio of PVP to Pd precursor being 5: (A, B) TEM images; (C, D) HRTEM images. The inset in (B) gives the TEM image of a 
titled triangular nanoplate showing one of its side faces. The inset of (C) shows a schematic drawing of the triangular nanoplate, where the red and green colors represent the $\{100\}$ and $\{111\}$ facets, respectively. Reprinted with permission from reference ${ }^{55}$. Copyright (2005) American Chemical Society.

Harada and Cong have recently described the synthesis of PdNPs by a MW-assisted polyol methodology, using EG or Gly as polyol, under continuous flow regime at $471 \mathrm{~K}$ (for EG) and $523 \mathrm{~K}$ (for glycerol) for about $10 \mathrm{~min}$. (Figure 5) ${ }^{56} \mathrm{~A}$ comparative study using both continuous flow-type and batch-type reactors for the synthesis of PdNPs (and also Rh, Ru and Pt NPs) in EG and Gly has proven the feasibility of the scale-up MNPs synthesis under flow conditions, as evidenced by TEM and EXAFS analyses of the structures obtained with both types of reactors. However, the authors do not describe the reaction atmosphere employed in their synthetic protocol.

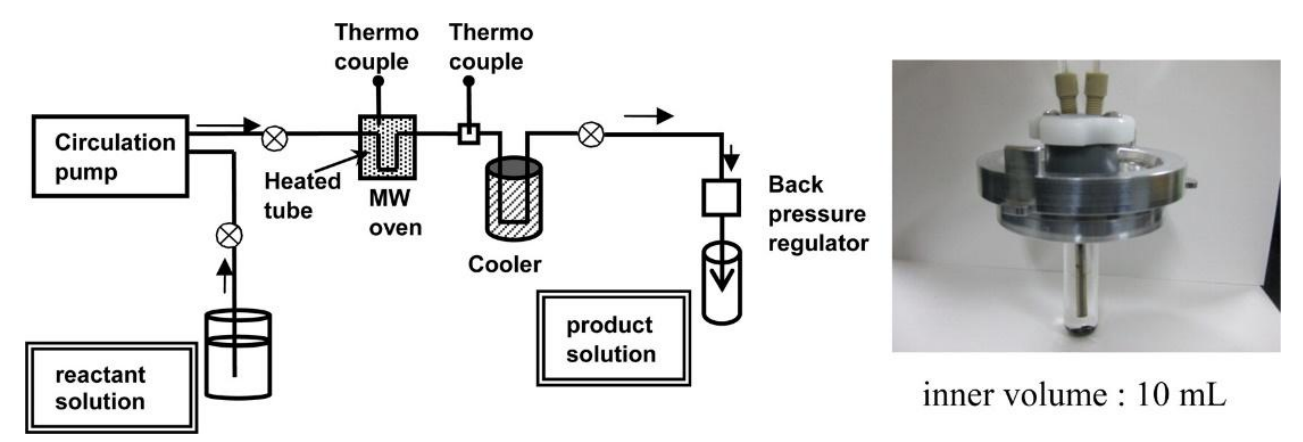

Figure 5. Illustration of the continuous-flow reactor system for the production of metal nanoparticles (left). CEM continuous-flow cell (right). Reprinted with permission from reference $^{56}$. Copyright (2016) American Chemical Society.

Sonochemical synthesis of noble metal nanoparticles have attracted the attention of researchers, mainly due to the high temperatures (thousands of degrees) and high pressures (hundreds of 
atmospheres) generated inside the collapsing cavitation bubbles [for the pioneering work of synthesis of PdNPs in water by ultrasound activation see ref. ${ }^{57}$. Rehspringer and co-workers have studied the synthesis of PdNPs from Pd(NO 3$)_{2}$ in water using EG as reductant and PVP as capping agent. ${ }^{58}$ They obtained spherical NPs with small sizes (from 3.5 to $5.5 \mathrm{~nm}$ ), depending on the Pd(II)/PVP molar ratio: the ratio increase (from $0.13 \times 10^{-3}$ to $0.53 \times 10^{-3}$ ) led to a slight mean diameter increase together with a decrease in number of NPs; for the highest Pd(II) content (more than $2.5 \times 10^{-3} \mathrm{~mol}$ ), aggregates of small NPs were observed.

Alternatively, Lamaty et al. have described in situ formation of spherical PdNPs of $7 \pm 1,9 \pm 2$ and $10 \pm 3 \mathrm{~nm}$ for catalytic Heck reactions (see section 4.4.) using $5 \mathrm{~mol} \% \mathrm{Pd}(\mathrm{OAc})_{2}, \mathrm{NaHCO}_{2}(0.2$ equiv.) as reducing agent in PEG1100, PEG2000 and PEG3400, respectively, under ball-milling $(30 \mathrm{~Hz})$ conditions and in the absence of phosphine ligands, respectively. ${ }^{59}$ Notably, in the absence of aryl halide and acrylate substrates, the reaction led to agglomeration.

\subsubsection{Glycerol and other polyols}

Coming from biomass and also obtained as the main coproduct ( $c a .10 \mathrm{wt} \%)$ in the biodiesel production by transesterification of vegetable oils, glycerol has caused a great interest in its applications, due to both economic and environmental standpoints. ${ }^{60}$ Its low-cost, non-toxicity, high boiling point $\left(290{ }^{\circ} \mathrm{C}\right)$, negligible vapor pressure $(<1 \mathrm{mmHg}$ at $293 \mathrm{~K})$, high solubilizing ability for organic and inorganic compounds (dielectric constant: 44.38 at $25{ }^{\circ} \mathrm{C}$; dipole moment: 2.68 D at $30-50^{\circ} \mathrm{C}$ ), and low miscibility with other organic solvents such as ethers and alkanes, ${ }^{61}$ constitute outstanding properties towards its use as solvent for applications in synthesis and catalysis. ${ }^{28,29,62-64}$ However, in the preparation of metal-based nanoparticles following a polyol methodology, glycerol has been much less used than EG. The work reported by Ratna's group in 
1994 can be considered as a pioneering research in this context. ${ }^{65}$ They used neutral glycerol monooleate acting as co-solvent, reducing agent, and stabilizer at $20-25{ }^{\circ} \mathrm{C}$ for the synthesis of PdNPs (using $\mathrm{Na}_{2}\left[\mathrm{PdCl}_{4}\right]$ as metal precursor in water), taking advantage of the bicontinuous cubic phase of glycerol monooleate, which generates a 3D network of channels; this approach led to spherical PdNPs exhibiting a mean diameter of $3.34 \pm 0.86 \mathrm{~nm}$. The authors do not report the use of inert atmosphere to carry out their experiments.

In 2011, Park and co-workers studied the shape-controlled synthesis of PdNPs in a mixture of glycerol- $\mathrm{H}_{2} \mathrm{O}$ with $\mathrm{PVP}$ as stabilizer at $100{ }^{\circ} \mathrm{C}$ by reduction of $\mathrm{Na}_{2}\left[\mathrm{PdCl}_{4}\right]$, obtaining triangular and hexagonal plates, together with decahedron as evidenced by field-emission transmission electron microscopy (FE-TEM) (Figure 6). ${ }^{66}$ This anisotropic growth was also observed by Grace and Pandian applying microwave activation instead of conventional heating up to 290 ${ }^{\circ} \mathrm{C} .{ }^{67}$ Neither of these two references describe the use of inert atmosphere to carry out the syntheses. 

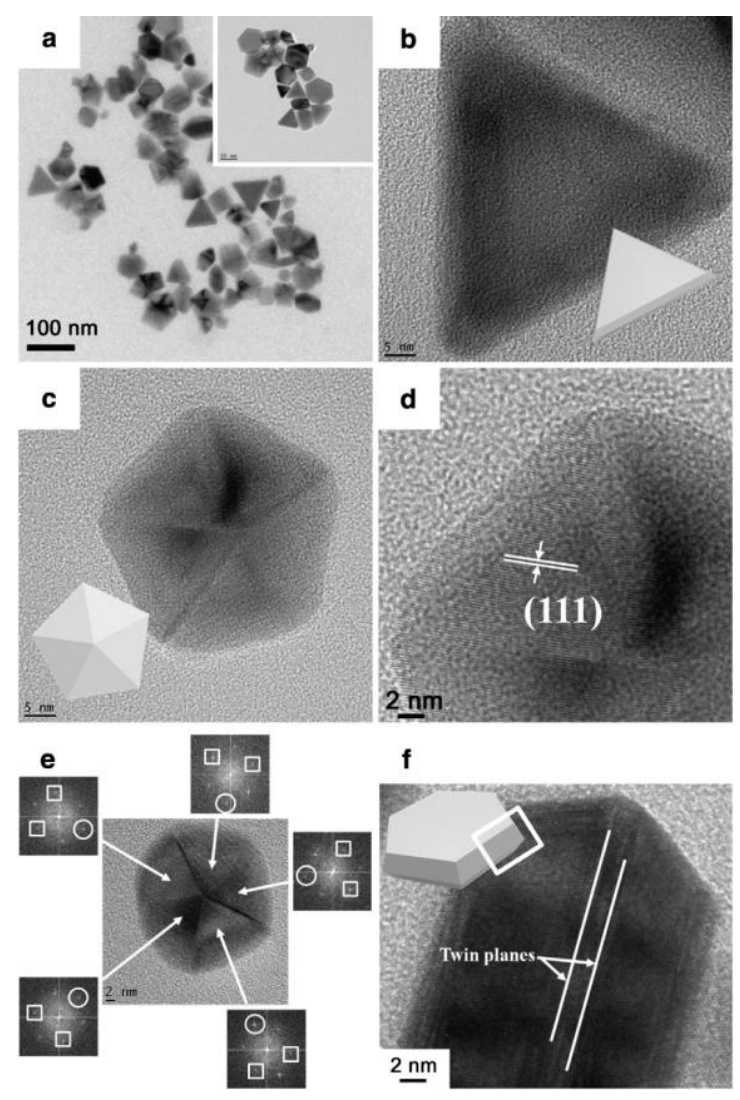

Figure 6. (a) FE-TEM and (b-c) HR-TEM micrographs of PdNPs synthesized in glycerol acting as reducing agent and PVP as stabilizer; (d) continuous lattice fringes to the decahedral PdNPs containing $\{111\}$ facets; (e) HR-TEM and FFT images of the decahedral PdNPs along the fivefold symmetry axis (insets indicate the FFT pattern of $\{111\}$ facet $(\square)$ and $\{100\}$ facet (o)); (f) HR-TEM image of PdNPs containing $\{111\}$ twin places parallel to the top and bottom faces of the hexagonal plate shape. Reproduced with permission from Elsevier, ${ }^{66}$ license number 4558831417480.

Varma and co-workers reported the effect of stabilizers such as PVP, CTAB and SDS on the shape and dispersion of noble metal NPs synthesis ( $\mathrm{Au}, \mathrm{Pt}, \mathrm{Pd})$ under MW irradiation at $100{ }^{\circ} \mathrm{C}$ (unspecified reaction atmosphere) ${ }^{68}$ For Pd, in the absence of any stabilizer, PdNPs of $c a .10 \mathrm{~nm}$ were obtained, without observing any variation in the presence of SDS. However, the addition of 
PVP or CTAB led to smaller and better dispersed PdNPs, although in CTAB the PdNPs tended to aggregate (Figure 7).
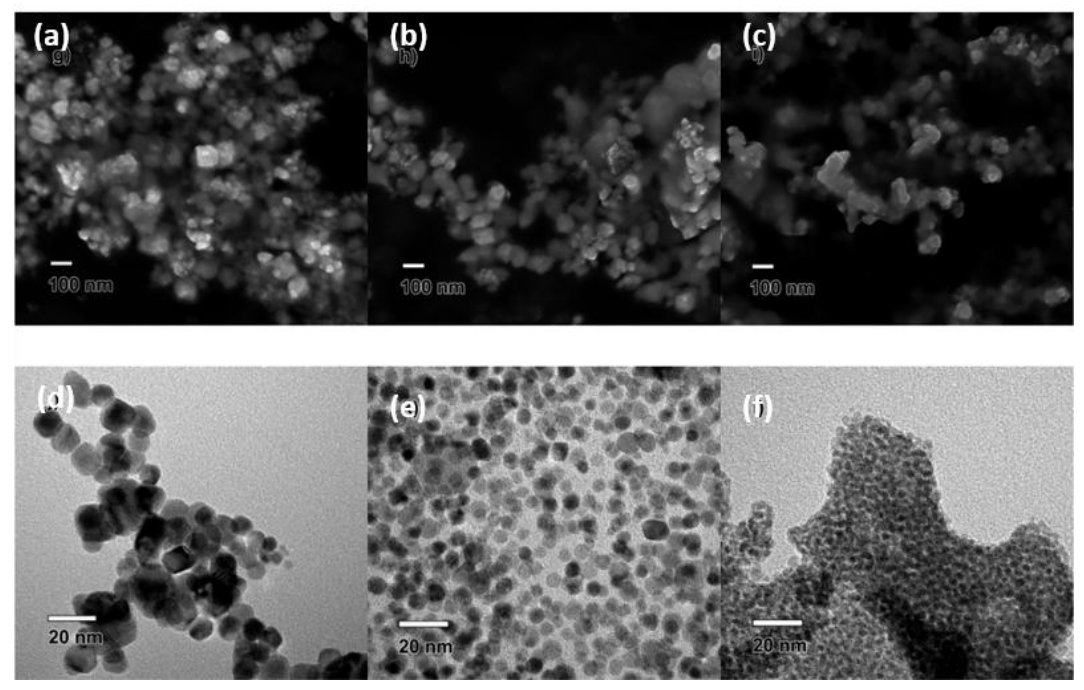

Figure 7. (a) SEM micrograph of PdNPs at 2 min. (b) SEM micrograph of PdNPs at 30 min. (c) micrograph of PdNPs at 2 min with SDS. (d) TEM micrograph of PdNPs at 2 min. (e) TEM micrograph of PdNPs at 2 min with PVP. (f) TEM micrograph of PdNPs at 2 min with CTAB. Adapetd with permission from reference. ${ }^{68}$ Copyright (2013) American Chemical Society.

Based on the idea of Ratna, ${ }^{65}$ O'Sullivan and co-workers developed liposomal nanoreactors for the preparation of PdNPs in glycerol and Ar gas bubbling at $25{ }^{\circ} \mathrm{C} .{ }^{69}$ For that purpose, PdNPs were prepared by encapsulation of $\mathrm{Na}_{2}\left[\mathrm{PdCl}_{4}\right]$ with glycerol in nanosized liposomes, which were generated from zwitterionic or charged lipids (Figure 8). Small (ca. $2 \mathrm{~nm}$ ) and well-dispersed PdNPs were obtained with 1,2-dioleoyl-sn-glycero-3-phosphor-rac-(1-glycerol) (DOPG), derived from glycerol. Raman spectra evidenced the presence of both glycerol and phospholipid in the composition of PdNPs, taking advantage of their surface-enhanced Raman scattering (SERS) properties. $^{70}$ 

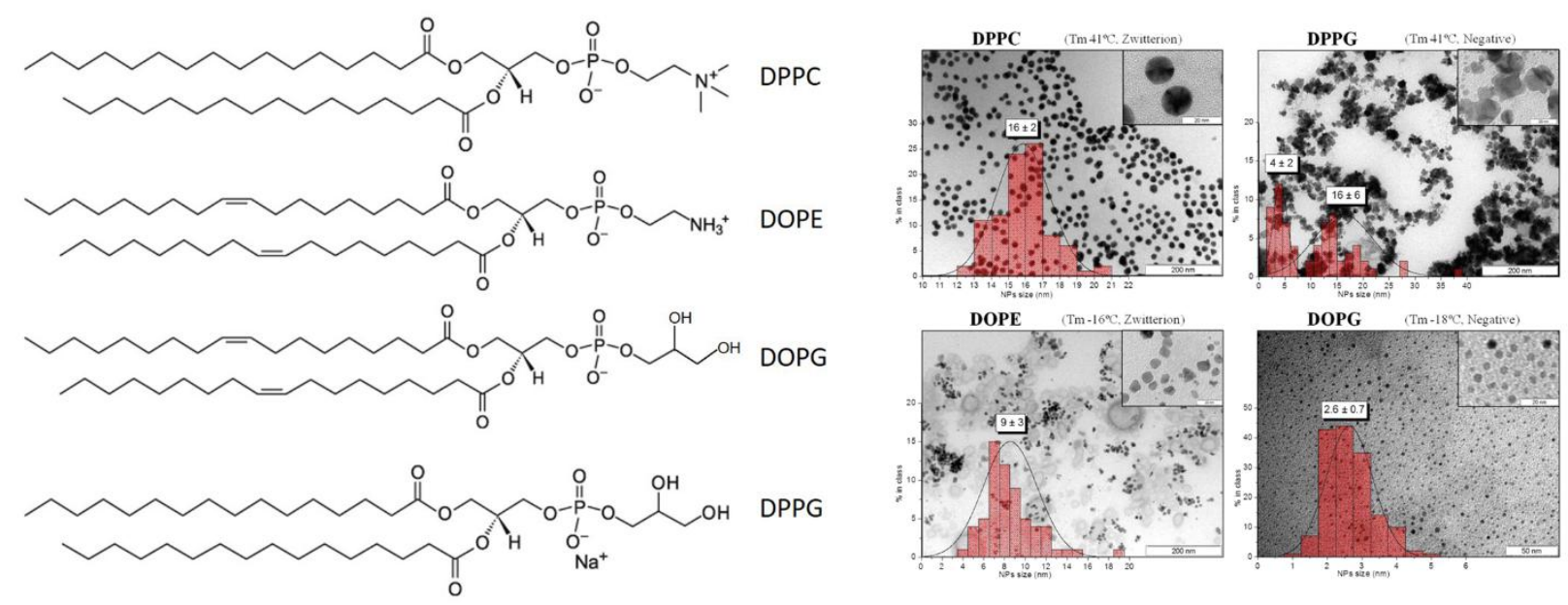

Figure 8. Structure of lipids employed for the nanoreactor study: 1,2-dipalmitoyl-sn-glycero-3phosphocholine (DPPC), 1,2-dioleoyl-sn-glycero-3-phosphoethanolamine (DOPE), ,2-dioleoylsn-glycero-3-phosphor-rac-(1-glycerol) (DOPG), and 1,2-dipalmitoyl-sn-glycero-3-phospho-rac(1-glycerol) (DPPG) and 1 (left). TEM images of PdNPs synthesized inside the different liposomes with the corresponding size distribution graphs; in the case of DPPG there are clearly two populations of NPs. Reprinted with permission from reference. ${ }^{69}$ Copyright (2013) American Chemical Society.

In our group, we were interested in the synthesis of metal-based NPs for applications in catalysis (couplings, hydrogenations, hydrodehalogenations, carbonylations... including tandem processes), in neat glycerol under base-free conditions, using molecular hydrogen as reducing agent, with the aim of avoiding any transformation of the solvent during the synthesis; besides palladium, NiNPs, ${ }^{71} \mathrm{Cu}_{2} \mathrm{O}$ NPs, ${ }^{72}$ and $\mathrm{CuNPs}^{73}$ were also prepared following this strategy at 100 ${ }^{\circ} \mathrm{C}, 100^{\circ} \mathrm{C}$ and $120^{\circ} \mathrm{C}$, respectively. Concerning monometallic PdNPs, we synthesized a number of nano-materials differing in the nature of the stabilizer. Thus, PdNPs were prepared from Pd salts and organometallic precursors, namely, $\mathrm{Pd}(\mathrm{OAc})_{2},\left[\mathrm{PdCl}_{2}(\mathrm{COD})\right],[\mathrm{Pd}(\mathrm{ma})(\mathrm{nbd})]$, and 
$\left[\mathrm{Pd}_{2}(\mathrm{dba})_{3}\right]$ (COD: 1,5-cyclooctadiene, ma: maleic anhydride, nbd: norbornadiene, dba: dibenzylidenacetone) in the presence of glycerol-soluble phosphines such as trisodium 3,3',3phosphinetriyltribenzenesulfonate (TPPTS ${ }^{74,75}$ and 1,3,5-triaza-7-phosphaadamantane (PTA) ammonium derivatives at $60^{\circ} \mathrm{C} .^{76}$ The as-prepared PdNPs were fully characterized, specifically in solution by XPS and Electron Microscopy techniques thanks to the negligible vapor pressure of glycerol (Figure 9).

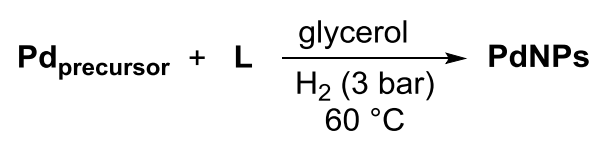

$\mathbf{P d}_{\text {precursor }}=\mathrm{Pd}(\mathrm{OAc})_{2},\left[\mathrm{PdCl}_{2}(\mathrm{cod})\right],[\mathrm{Pd}(\mathrm{ma})(\mathrm{nbd})],\left[\mathrm{Pd}_{2}(\mathrm{dba})_{3}\right]$ $(\operatorname{cod}=1,5$-cyclooctadiene, $\mathrm{ma}=$ maleic anhydride, $\mathrm{nbd}=$ norbornadiene, $\mathrm{dba}=$ dibenzylidenacetone

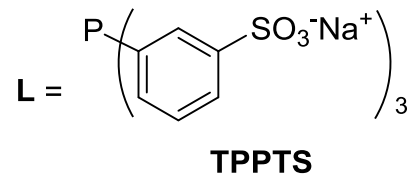

TPPTS

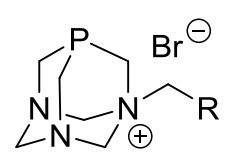

PTA-R

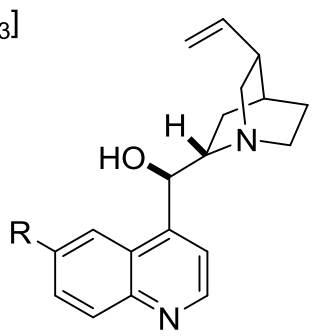

$\mathrm{R}=\mathrm{H}$, Cinchonidine $(\mathrm{CD})$

$\mathrm{R}=\mathrm{OMe}$, Quinine $(\mathbf{Q N})$

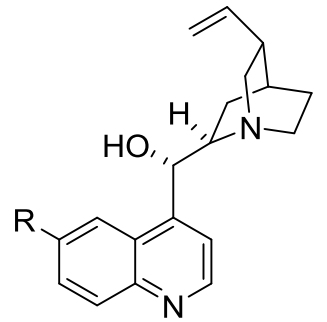

$\mathrm{R}=\mathrm{H}$, Cinchonine $(\mathbf{C N})$

$\mathrm{R}=\mathrm{Ph},\left(4-\mathrm{CH}_{2} \mathrm{OH}\right)-\mathrm{C}_{6} \mathrm{H}_{4}$, 2-naphthyl, (4-SMe)- $\mathrm{C}_{6} \mathrm{H}_{4}$, (4-OBn)- $\mathrm{C}_{6} \mathrm{H}_{4},\left(4-\mathrm{OC}_{12} \mathrm{H}_{25}\right)-\mathrm{C}_{6} \mathrm{H}_{4}$
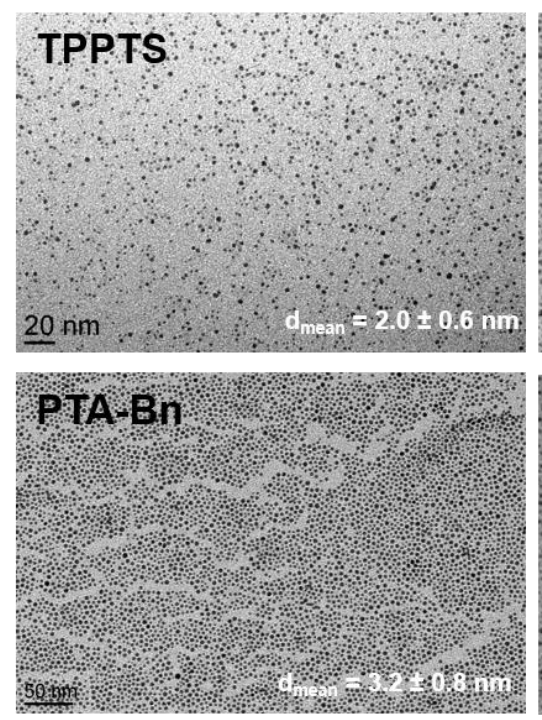
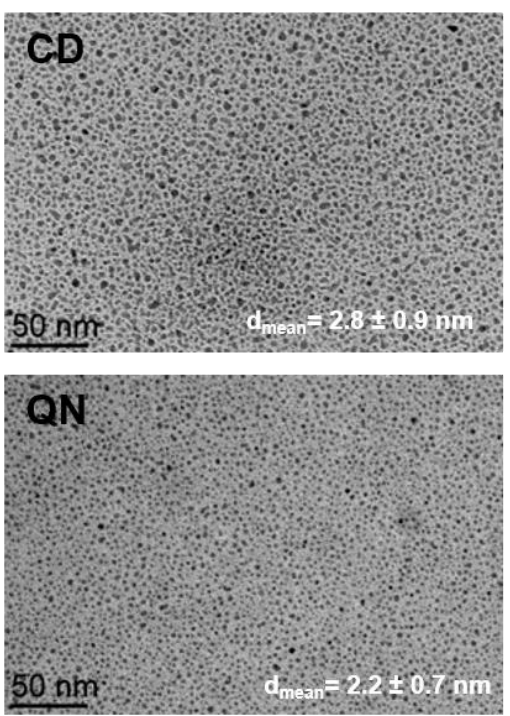
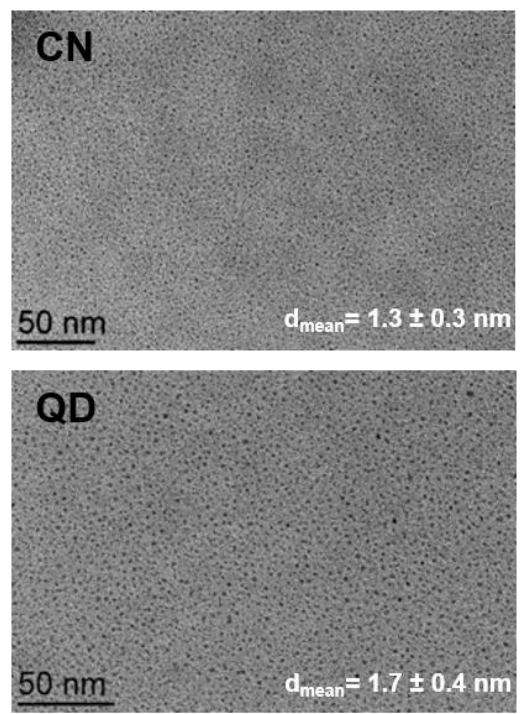
Figure 9. Synthesis of PdNPs in neat glycerol from $\mathrm{Pd}(\mathrm{II})$ and $\mathrm{Pd}(0)$ precursors under molecular hydrogen pressure using different types of stabilizers (top). TEM images recorded in glycerol for some of these PdNPs (bottom). ${ }^{71,74,76}$

The as-prepared PdNPs were analyzed by (HR)TEM showing the formation of highly dispersed small nanoparticles; EDS analyses evidenced the presence of the corresponding stabilizer on the isolated nanoparticles. With the aim of checking the coordination of TPPTS at the metallic surface in glycerol, the synthesis and ${ }^{31} \mathrm{P}$ NMR monitoring of TPPTS-stabilized PdNPs was carried out at NMR tube scale (Figure 10). In the absence of molecular hydrogen, the reaction between $\mathrm{Pd}(\mathrm{OAc})_{2}$ and TPPTS at $60{ }^{\circ} \mathrm{C}$ in glycerol afforded the formation of palladium phosphine complexes (signals in the range $22-35 \mathrm{ppm})$. After adding $\mathrm{H}_{2}(3 \mathrm{bar})$, phosphorus signals almost disappeared due to the formation of PdNPs. ${ }^{77,78}$ Given the high affinity of sulphur compounds for late transition metal surfaces, dodecanethiol was then added to the mixture and TPPTS could be quantitatively released from the surface as detected by ${ }^{31} \mathrm{P}$ NMR. ${ }^{77}$ 


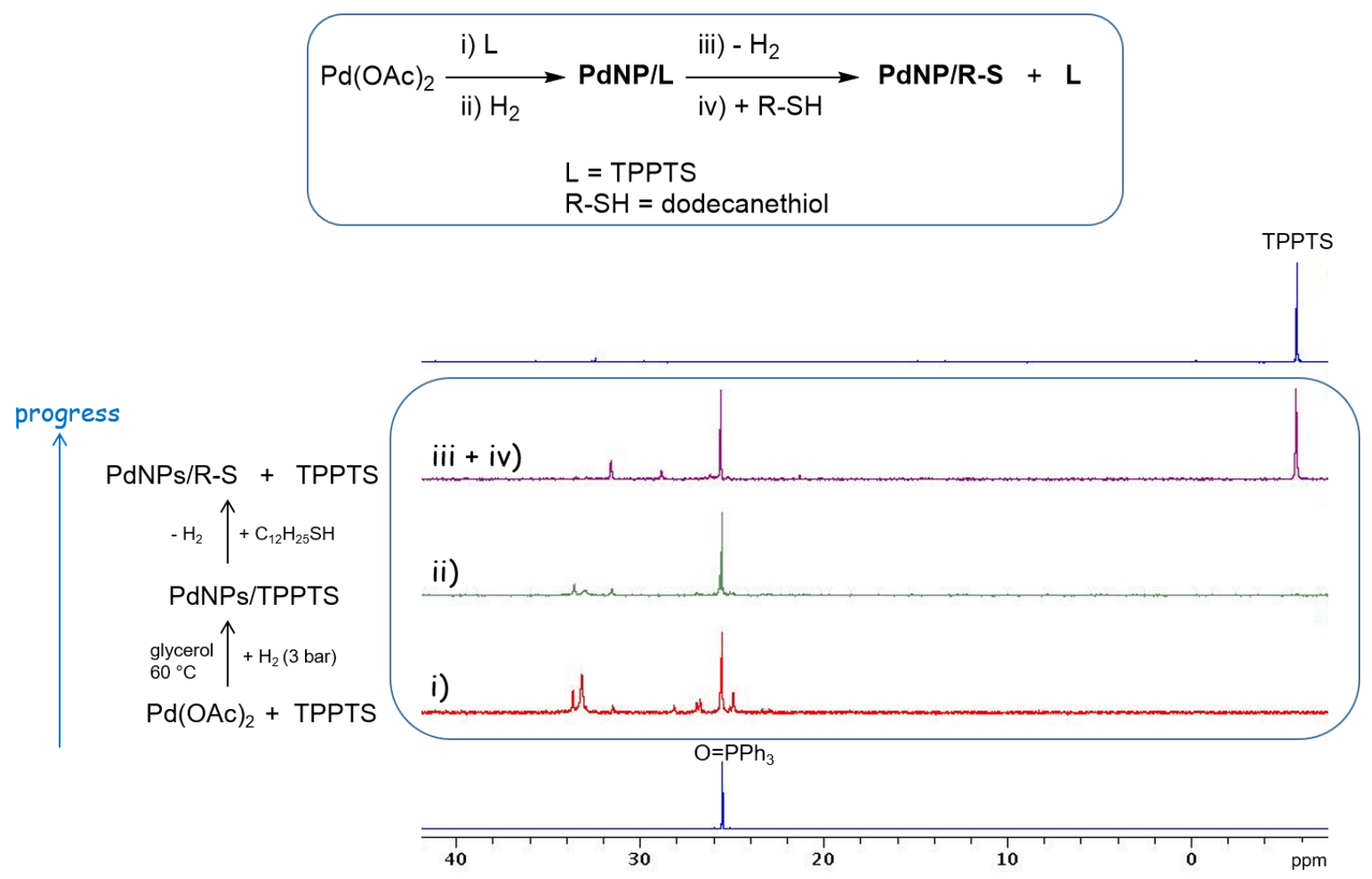

Figure 10. ${ }^{31} \mathrm{P}$ NMR monitoring of the formation of PdNPs stabilized by TPPTS in glycerol under 3 bar $\mathrm{H}_{2} \cdot{ }^{31} \mathrm{P}$ NMR spectra $(121 \mathrm{MHz}, 298 \mathrm{~K})$ starting from $\mathrm{Pd}(\mathrm{OAc})_{2}$ and TPPTS in 0.7 $\mathrm{mL}$ of glycerol and $0.3 \mathrm{~mL}$ of $\mathrm{D}_{2} \mathrm{O}$, using $\mathrm{O}=\mathrm{PPh}_{3}$ in glycerol/ $\mathrm{D}_{2} \mathrm{O}$ as external standard (placed in an external capillary; 1 equiv. in relation to TPPTS); top spectrum corresponds to neat TPPTS and bottom spectrum corresponds to neat triphenylphoshine oxide. ${ }^{74}$

With the aim of modifying the coordination mode of the ligand at the metal surface, cinchonabased alkaloids were also used as stabilizers (Figure 9). ${ }^{79}$ In contrast to the strong interactions between P-donor ligands and metal surface, ${ }^{80}$ mainly by $\sigma$-dative Pd-P bonds, cinchona ligands can interact with the surface through both $N$-quinoline $\sigma$ bonding or by $\pi$ interaction with the quinoline aromatic ring. ${ }^{81,82}$ Using quinidine as stabilizer, small $(1.4 \pm 0.3 \mathrm{~nm})$ and welldispersed PdNPs were obtained; PXRD and HR-TEM analyses evidenced the formation of fcc 
PdNPs. XPS spectra in glycerol confirmed the exclusive presence of $\operatorname{Pd}(0)$; curiously, in the nitrogen region, two signals were observed, in contrast to one signal observed for PdNPs at solid state (isolated by centrifugation) and for the free ligand. This behavior could be explained because two types of quinidine molecules are present in glycerol solution, those adsorbed at the metal surface and those solvated by glycerol as evidenced by ${ }^{15} \mathrm{~N}$ HMBC NMR experiments.

For a summary of monometallic Pd-based NPs synthetic conditions and characterization outcomes, see Table S1 in the Supporting Information.

\subsection{Palladium bimetallic nanoparticles}

Bimetallic nanoparticles represent a noteworthy type of nanomaterials due to their unique physico-chemical properties in relation to their corresponding monometallic counterparts. These distinctive features are mainly due to their composition and structure, causing specific electronic effects and in particular singular catalytic behavior, which is distinguished by the synergy between both metals. ${ }^{83-85}$ Furthermore, the miscibility of the metals constituting Pd-based BMNPs is a relevant parameter with a direct impact on their structure. ${ }^{86,87}$ The use of alternative solvents for the synthesis of BMNPs is especially attractive in order to control the reduction rate of the metal precursors with the consequent impact on nucleation, growth, shape and structure of the polymetallic nanoparticles. ${ }^{88}$

After the pioneering work of Silvert et al. concerning the synthesis of PdAg nanoalloys by polyol methodology using EG as solvent, ${ }^{89}$ a plethora of contributions of palladium bimetallic nanoparticles (BMNPs) have been described in the literature, mostly containing noble metals, such as gold, platinum and ruthenium, but also BMNPs including first-row transition metals, such as iron, cobalt, nickel and copper. Here, we describe the main works reported in the 
literature in relation to controlled syntheses affording well-defined PdM nanoparticles (where M is a metal other than Pd) using polyols as solvents, including their characterization and mechanistic approaches; in the main part of these contributions, the polyol acts also as reducing agent. Most works concern AuPd and PdPt nanoparticles mainly showing both core-shell and alloy structures. In the most cases, the BMNPs are prepared under batch conditions by successive or simultaneous reduction of the corresponding metal salts. Depending on reaction conditions, simultaneous co-reduction of the metal precursors can lead to core-shell structures and sequential addition of precursors can give alloys. Other BMNPs arrangements have been also observed, such as cluster-in-cluster. The composition and distribution of the elements in the nanoparticles is often assessed by scanning transmission electron microscopy combined with X-ray energy dispersive spectroscopy (STEM-EDS), together with data from other techniques such as PXRD, cyclic voltammetry, including theoretical calculations.

2.2.1. Palladium bimetallic nanoparticles containing $4 \mathrm{~d}$ and $5 \mathrm{~d}$ transition metals.

\section{Gold}

Gedanken and Harpeness reported the first synthesis of $\mathrm{Au}(\mathrm{core}) \mathrm{Pd}($ shell) nanoparticles by coreduction of metal salts in EG under microwave conditions. ${ }^{90}$ Thermodynamically, the reduction of $\mathrm{Au}(\mathrm{III})$ ions is favored in relation to $\mathrm{Pd}(\mathrm{II})$ ions [the reduction potential of $\mathrm{Au}(\mathrm{III})$ is more positive than that of $\mathrm{Pd}(\mathrm{II})]$ and additionally, the reduction of $\left[\mathrm{AuCl}_{4}\right]^{-}$species is faster than $\left[\mathrm{PdCl}_{4}\right]^{2-}$ ions, favoring the formation of Au clusters which act as seeds for the growth of the Pd layer; this behavior was also observed using glycerol as solvent. ${ }^{91}$ From these results, the plausible catalytic role of the initial AuNPs for the reduction of Pd(II) ions cannot be ruled out. Actually, Yang and co-workers have proved that the gold-based seeds promote the reduction of 
$\operatorname{Pd}(\mathrm{II})$ species leading to the formation of $\mathrm{Au}($ core)-Pd(shell) BMNPs under smooth reduction conditions. ${ }^{92}$ José-Yacamán and co-workers prepared AuPd nanoparticles by sequential reduction of $\mathrm{Pd}(\mathrm{II})$ followed by $\mathrm{Au}(\mathrm{III})$ ions in EG and in the presence of PVP under conventional heating. ${ }^{93}$ The as-prepared BMNPs showed a complex structure above a critical size ( $c a$. larger than $5 \mathrm{~nm}$ ), which led to three-layer nanoparticles. As evidenced by STEM-EDS line scanning analyses, the core is constituted by an alloy, surrounded by an Au-rich layer and followed by a Pd-rich external layer (Figure 11). Studies of phase diagrams at nanoscale indicate that these diagrams become more complex as the size is diminished. ${ }^{94}$

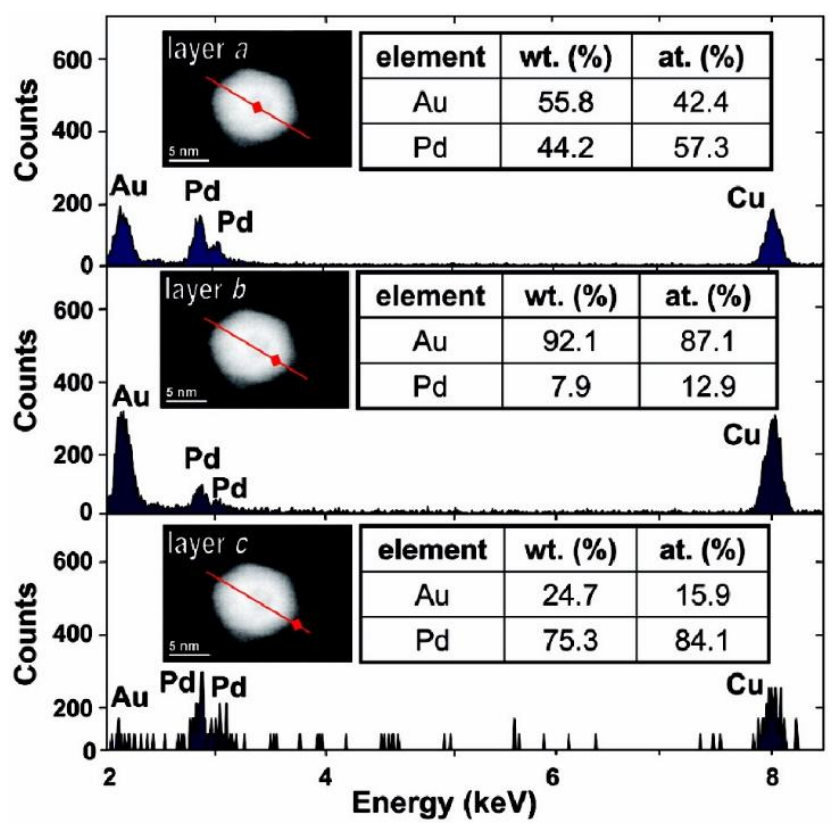

Figure 11. Quantitative STEM-EDS line scanning analyses for AuPd BMNPs prepared in glycerol. Reprinted with permission from reference ${ }^{93}$. Copyright (2007) American Chemical Society.

Rosales and co-workers prepared AuPd alloys (Au and Pd are miscible) by successive addition of metal precursor in EG using PVP as capping agent under conventional thermal activation, 
exhibiting different compositions $\left(\mathrm{AuPd}, \mathrm{Au}_{3} \mathrm{Pd}, \mathrm{AuPd}_{3}\right) .{ }^{95}$ In this work, which combines experimental and theoretical approaches, it was proven that the different nano-alloys showed a rough surface with several types of surface defects (kinks, edges, vacancies), with a truncated octahedron morphology; this behavior is in contrast to monometallic nanoparticles for which icosahedra with defect-free surfaces were obtained, structures corresponding to low energy shapes as proven by the authors by theoretical calculations (molecular dynamics cooling process methodology).

Silver

Kim et al. described for the first time AgPd nanoalloys synthesized by a polyol approach for hydrogen permeation purposes. ${ }^{96}$ They were prepared by co-reduction of the corresponding metal salts $\left(\mathrm{Pd}\left(\mathrm{NO}_{3}\right)_{2}\right.$ and $\left.\mathrm{AgNO}_{3}\right)$ in $\mathrm{EG}$ at $190{ }^{\circ} \mathrm{C}$, in the absence of any other stabilizer, leading to the formation of aggregates. Powder XRD analyses evidenced the presence of cubic small crystallites with a lattice parameter $(a=3.942 \AA)$, between that of Pd $(3.889 \AA)$ and $\mathrm{Ag}$ $(4.085 \AA)$, indicating the formation of an alloy. STEM coupled with EDS line-scanning analyses showed the alloy arrangement and the Ag:Pd ratio of $c a .1: 3$, in agreement with the ratio used in the synthesis. The addition of stabilizers leads to well-dispersed alloyed AgPd NPs; therefore, well-defined AgPd NPs were obtained by sequential addition of both metals in DEG using hexadecylamine, ${ }^{97}$ as well as in EG using PVP as capping agent. ${ }^{98}$ In this latter case, spherical AgPd NPs were observed by HAADF-STEM analysis (mean diameter ca. $9.1 \mathrm{~nm}$ ); EDS mappings evidenced the formation of alloys (Figure 12). 

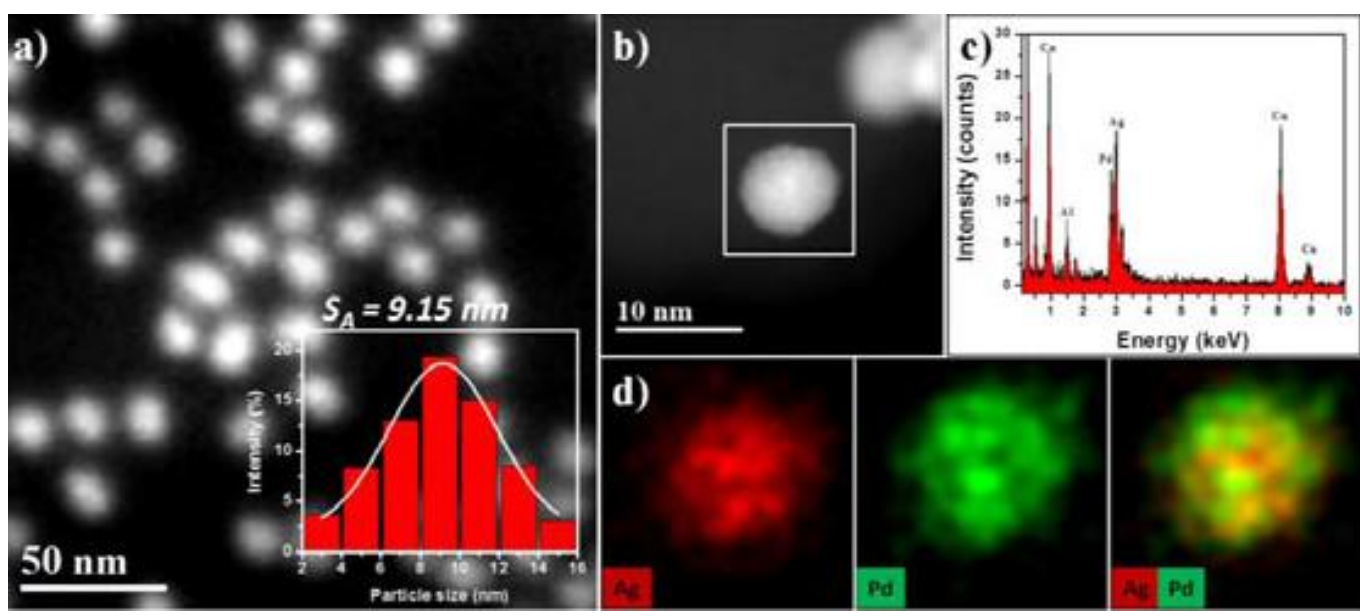

Figure 12. a) HAADF-STEM image of the AgPd BMNPs and its corresponding size distribution grapht; b) HAADF-STEM image of a single AgPd nanoparticle; c) EDS analysis; d) elemental mapping of Ag, Pd, and AgPd. Reprinted with permission from Cambridge University Press, ${ }^{98}$ license number 4558840711614.

\section{Platinum}

Jurczakowski and co-workers prepared PdPt nanoparticles in neat EG by co-reduction of $\mathrm{PdCl}_{2}$ and $\mathrm{K}_{2} \mathrm{PtCl}_{4}$ in the absence of any other reagent, leading to nanoparticles highly aggregated as proven by TEM analyses. ${ }^{99}$ These materials were used to study the spontaneous segregation of PdPt nanoalloys, mainly evidenced by the enhanced hydrogen solubility under ambient conditions together with XPS studies, proving a higher segregation trend than their bulk counterparts. These experimental results confirm the theoretical DFT studies reported by Barcaro et al. concerning the multi-shell segregation of PdPt nano-alloys. ${ }^{100}$ In agreement with both experimental and theoretical approaches, the works described below in relation to the synthesis and characterization of well-defined PdPt nanoparticles in polyol media correspond to segregate phases (core-shell, cluster-in-cluster). 
The synthesis of $\mathrm{Pt}($ core $) \operatorname{Pd}($ shell) nanoparticles has been mostly reported in the literature by applying the polyol methodology, according to a sequential addition of metal precursors. Moreover, the presence of small amounts of silver salt is required, which acts as a structuremodifying agent of the Pt nanocluster seeds as proven by Viet Long, Nogami and co-workers; ${ }^{101-}$ ${ }^{103}$ in general, silver is not detected in the final product, probably due to its precipitation or removal during the purification process. Following this strategy, José-Yacamán and co-workers have reported a deep characterization of $\mathrm{Pt}($ core $) \mathrm{Pd}($ shell) nanoparticles prepared in EG and using PVP as stabilizer. ${ }^{104}$ This time, they could observe a small amount of silver in the core. The nanoparticles obtained have cuboctahedral-octahedral and cuboctahedral-truncated tetrahedral core-shell morphology. Authors could confirm the presence of Pt core and Pd shell by HAADF-STEM images, STEM-EDS line scans and elemental maps of isolated nanoparticles (Figure 13). 


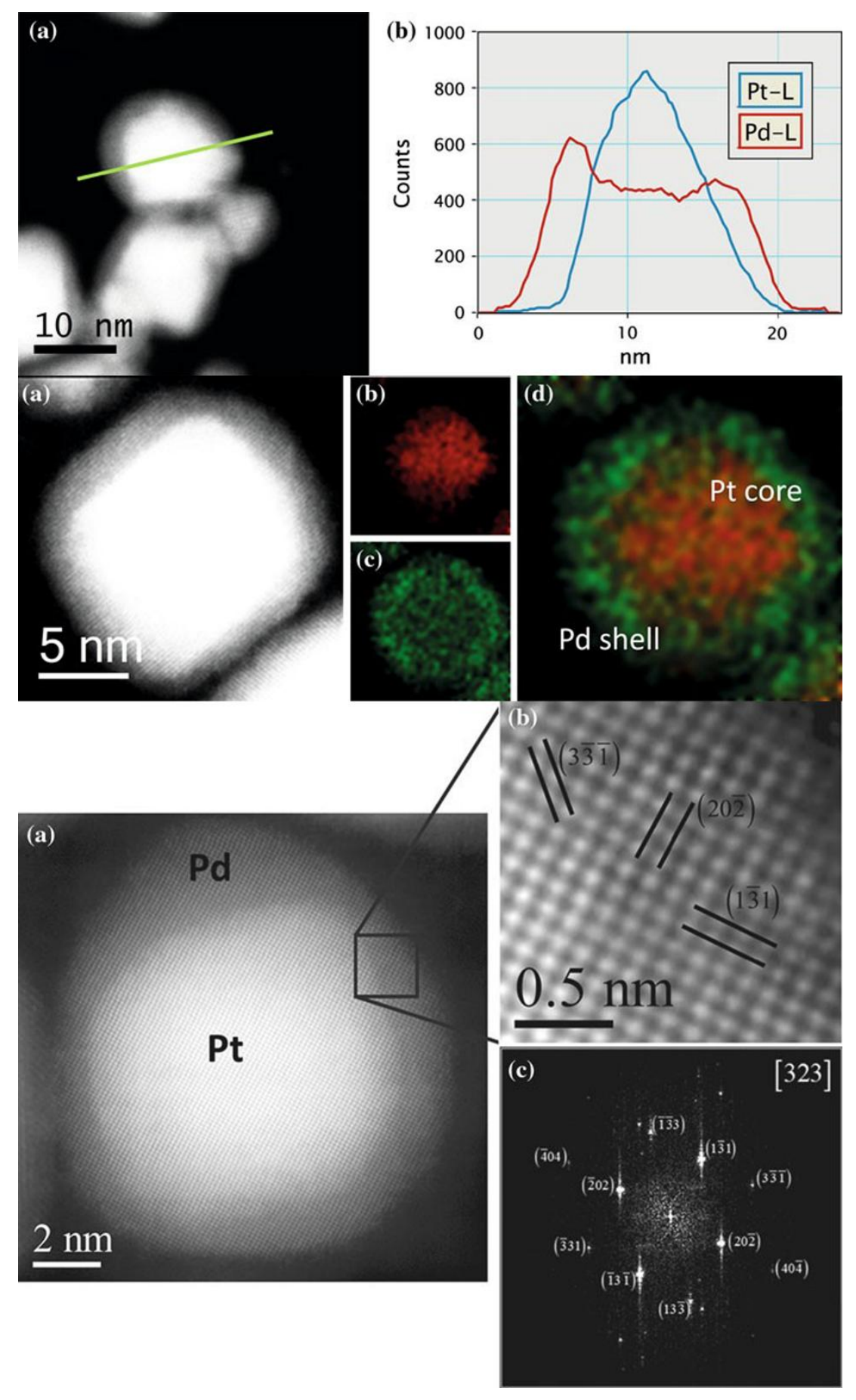

Figure 13. Top: STEM and EDS line scan analyses for $\operatorname{Pt}($ core)Pd(shell) nanoparticles: (a) HAADF-STEM image; (b) elemental line scan. Middle: (a) HAADF-STEM image of Pt(core)Pd(shell) nanoparticle; (b)-(d) STEM-EDS elemental maps: (b) Pt-L $\alpha$; (c)Pd-L $\alpha$; (d) Pt(core)Pd(shell) nanoparticle. Bottom: (a) HAADF-STEM image of Pt-Pd core-shell structure; 
(b) Zone indicated indicated by the rectangle showing the main crystalline planes; (c) FFT showing the [323] zone axis. Reprinted with permission from Springer Nature, ${ }^{104}$ license number 4558850402033.

$\mathrm{Pd}($ core $) \mathrm{Pt}($ shell) nanoparticles (mean size $6.5 \pm 0.6 \mathrm{~nm}$ ) have also been prepared using flow processes under microwave irradiation, in a sequential addition of metal precursors in EG or Gly and using PVP as stabilizer; ${ }^{105}$ EDS analysis evidenced the formation of a shell thickness of $c a$. $0.25 \mathrm{~nm}$ around the $\mathrm{Pd}$ core, which corresponds to one atomic layer of Pt. Harada and coworkers prepared PdPt nanoparticles showing a cluster-in-cluster structure, as proven by HRTEM, EDS and EXAFS, under both batch and continuous flow conditions. ${ }^{106}$ These arrangements were observed in both synthetic approaches under single-mode microwave irradiation, in EG or Gly and in the presence of PVP. However, under flow conditions and in contrast to batch ones, BMNPs were better dispersed showing smaller mean size, in particular using EG as solvent.

\section{Ruthenium}

$\mathrm{Ru}($ core $) \mathrm{Pd}($ shell) nanoparticles have been prepared in EG using PVP as stabilizer applying a sequential procedure, where firstly a $\mathrm{Ru}$ core nanocluster was generated from reduction of $\mathrm{RuCl}_{3}$ and then the palladium precursor was added forming a $\mathrm{Pd}$ layer around the former $\mathrm{Ru}$

nanoparticles. ${ }^{107}$ The as-prepared BMNPs showed a cylinder-like shape with a high density of terrace and kinks defects in the surface (Figure 14). These $\mathrm{Ru}($ core)Pd(shell) nanoparticles were then treated with a solution of $\mathrm{H}_{2} \mathrm{PtCl}_{6}$ in $\mathrm{EG}$; the surface defects of the $\mathrm{RuPd}$ nanoparticles were nucleation centers for promoting the growth of Pt clusters (Figure 14c). SAXS spectra corroborated the structure of the trimetallic $\mathrm{Pt} / \mathrm{Ru}($ core $) \mathrm{Pd}($ shell) nanoparticles. DFT calculations 
found that electrons are injected to Pt nanoclusters from the core-shell, electrons localized at the near Fermi level of the nanoparticles. The size-controlled synthesis of alloy RuPd nanoparticles is particularly challenging taking into account that palladium and ruthenium are immiscible at atomic level in the bulk state.

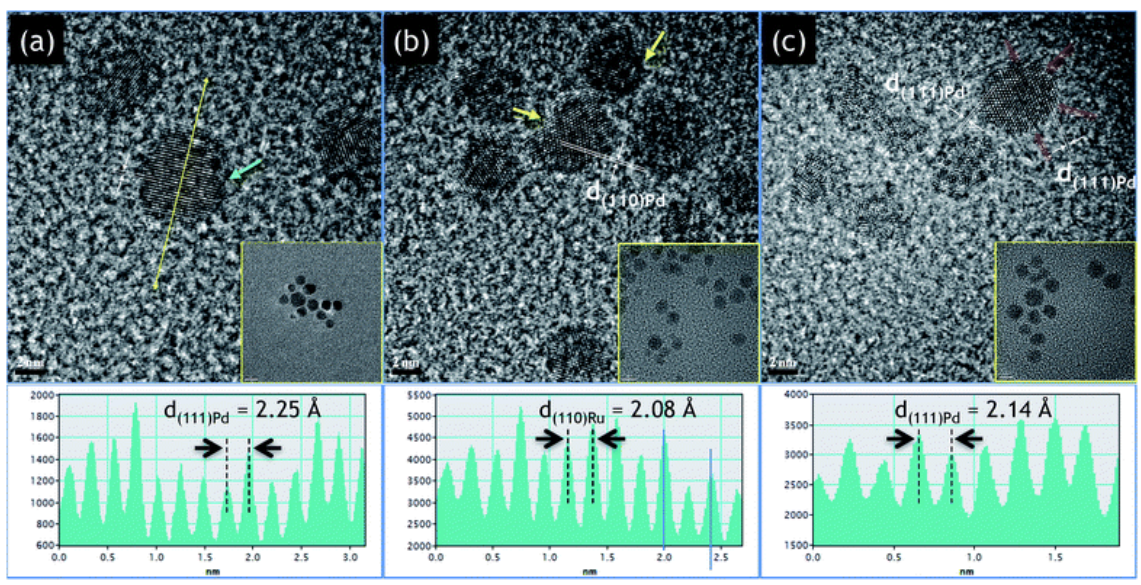

Figure 14. HRTEM micrographs of (a) $\mathrm{Pd}$, (b) $\mathrm{Ru}($ core) $\mathrm{Pd}$ (shell), and (c) $\mathrm{Pt} / \mathrm{Ru}$ (core) $\mathrm{Pd}$ (shell) nanoparticles. Reprinted with permission from Royal Society of Chemistry, ${ }^{107}$ license number 4558871121193.

Kusada and co-workers have prepared alloyed $\operatorname{Pd}_{x} \operatorname{Ru}_{1-\mathrm{x}}(\mathrm{x}=0.1,0.3,0.5,0.7,0.9)$ nanoparticles in TrEG by co-reduction of $\mathrm{Ru}(\mathrm{III})$ and Pd(II) salts in the presence of PVP that consisted of both fcc and hep structures, coexisting both phases in an individual nanoparticle (Figure 15). ${ }^{108}$ These nanomaterials exhibited higher catalytic behavior in the $\mathrm{CO}$ oxidation than the monometallic ruthenium nanoparticles. This type of nanoalloys has been also applied in the electrooxidation of formic acid and methanol. ${ }^{109,110}$ 
(a)

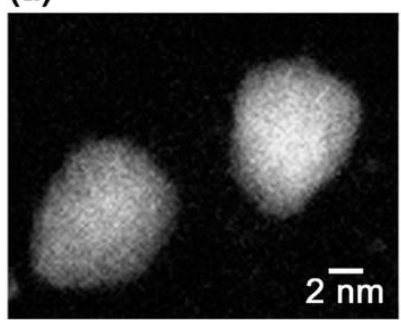

(c)

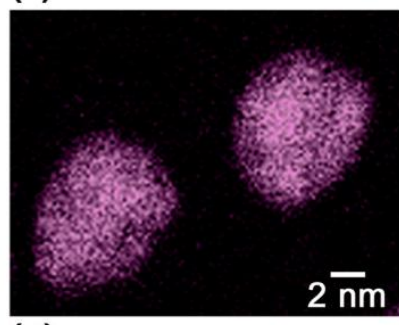

(e)

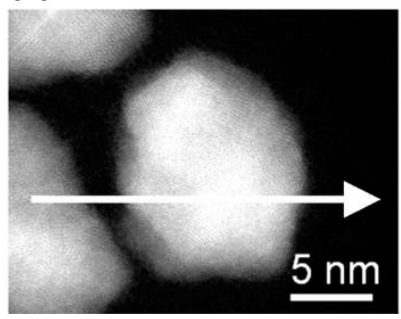

(b)

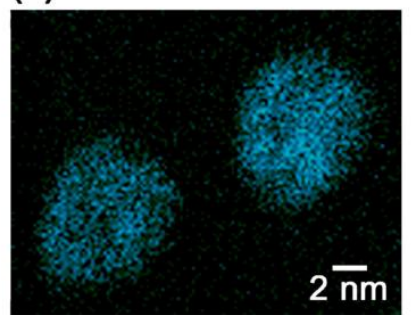

(d)

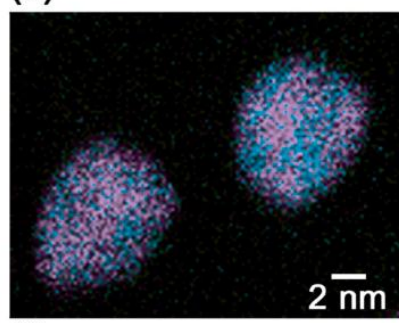

(f)

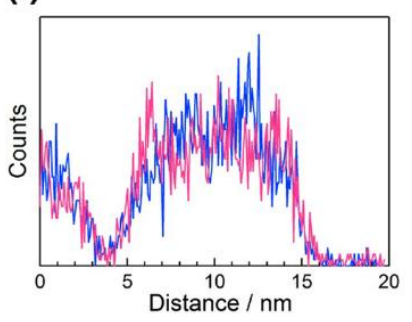

(a)

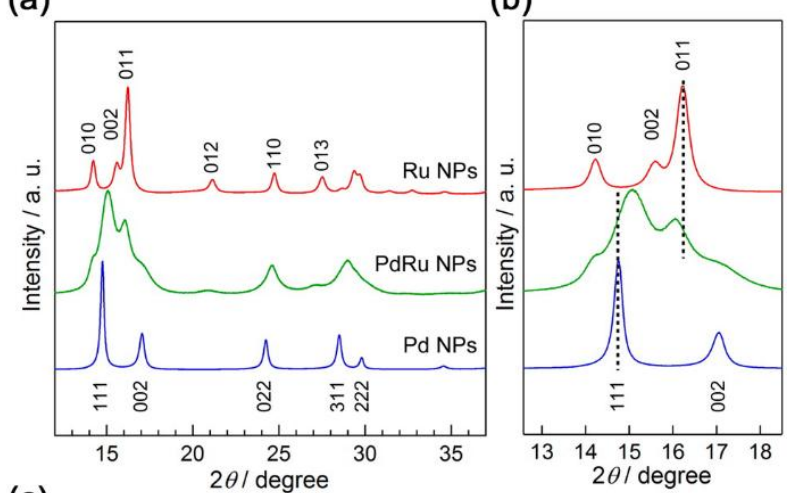

(c)

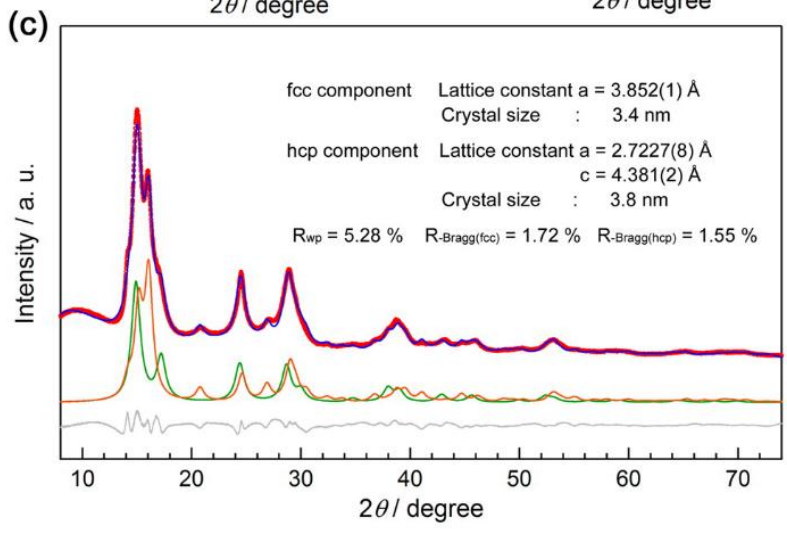

Figure 15. Left: (a) HAADF-STEM image, (b) Pd-L STEM-EDX map and (c) Ru-L STEMEDX map for $\mathrm{Pd}_{0.5} \mathrm{Ru}_{0.5}$ nanoparticles; (d) reconstructed overlay image of the maps shown in (b) and (c) (blue, Pd; red, Ru); (f) scan line profiles of $\mathrm{Pd}$ (blue) and $\mathrm{Ru}$ (red) for the $\mathrm{Pd}_{0.5} \mathrm{Ru}_{0.5}$ nanoparticle along the arrow shown in (e). Right: (a) synchrotron XRD analyse $\left(2 \theta=12^{\circ}-37^{\circ}\right)$ of $\mathrm{Ru}, \mathrm{Pd}$, and $\mathrm{Pd}_{0.5} \mathrm{Ru}_{0.5}$ nanoparticles at $303 \mathrm{~K}$; (b) $2 \theta=12.5^{\circ}-19^{\circ}$ region; (c) diffraction patterns of $\mathrm{Pd}_{0.5} \mathrm{Ru}_{0.5}$ nanoparticles (red circles) at $303 \mathrm{~K}$ and the corresponding calculated diffractogram (blue line); bottom lines show the difference profile (gray) and the fitting curves of the fcc (green) and hcp (orange) contributions (radiation wavelength $=0.57803(2) \AA$ ). Reprinted with permission from reference ${ }^{108}$. Copyright (2014) American Chemical Society.

As known, the control of structure and morphology of metal-based nanoparticles is crucial for establishing structure-property relationships. Therefore, it is essential to optimize all the 
parameters involved in the synthesis and to determine the corresponding kinetic profiles. The reduction rate of metal precursors helps to understand the mechanism involved in the formation of nanostructures. However the precursor conversion is rarely quantified, due to the difficulties to separate the formed nanoparticles from the unreacted starting material (see section 2.1.1.). ${ }^{45}$ With the aim of monitoring the synthesis of PdRu nano-alloys, Asano and co-workers have used a microflow reactor in order to achieve both rapid heating and a controlled short residence time. ${ }^{111}$ Working under semi-batch conditions, samples could be collected from the reactant solution at different times and analyzed by STEM-EDS and ICP-AES (local and bulk analysis technique, respectively). This monitoring revealed a slower reducing time for $\mathrm{Ru}(\mathrm{III})$ than for $\operatorname{Pd}(\mathrm{II})$; as the reaction time increases, PdRu nanoparticles become more homogeneous in atomic composition and their mean size increase. This latter fact indicates that particle growth is more dominant than nucleation, which only seems to proceed at the beginning of the injection. In addition, the change in the atomic distribution of the $\mathrm{Ru}$ on the time is probably related to an intraparticle solid state diffusion. When metal precursors were added in a sequential way, the resulting BMNPs were not alloyed as evidenced by XRD.

\section{Bismuth}

Interestingly, $\mathrm{Bi}_{2} \mathrm{Pd}$ cube-shaped nanoparticles, with diameters from 40 to $60 \mathrm{~nm}$, were prepared in neat TEG using $\mathrm{NaBH}_{4}$ as reducing agent, showing $\beta$ - $\mathrm{Bi}_{2} \mathrm{Pd}$ structure as evidenced by powder

X-ray diffraction. ${ }^{112}$ Moreover, the microwave-assisted polyol methodology was applied in the preparation of nanosized ternary $\mathrm{Pd}_{3} \mathrm{Bi}_{2} \mathrm{X}_{2}(\mathrm{X}=\mathrm{S}, \mathrm{Se})$ phases $(c a .50 \mathrm{~nm})$, using EG as solvent; microwave irradiation favors the dissolution of reagents, in particular in this case, elemental selenium. ${ }^{113}$ 
2.2.2. Palladium bimetallic nanoparticles containing $3 \mathrm{~d}$ transition metals.

Iron

Based on the methodology described by Sun et al. for the synthesis of FePt nanoparticles, ${ }^{114}$ Ohta and co-workers prepared FePd NPs by simultaneous reduction of $\operatorname{Pd}(\mathrm{acac})_{2}(\mathrm{acac}=$ acetylacetonate anion) and thermal decomposition of $\mathrm{Fe}(\mathrm{CO})_{5}$ in 1,2-hexadecanediol at high temperature $\left(280-300{ }^{\circ} \mathrm{C}\right)$, in the presence of tributylphosphine and adamatanecarboxylic acid or oleic acid as stabilizer; this methodology led to the formation of spherical NPs showing a narrow size distribution (mean diameter: $11 \pm 2 \mathrm{~nm}$ ). ${ }^{115}$ XRD analysis evidenced a disordered cubic structure and the shift observed of the (111) peak pointed to the formation of FePd alloy. By modifying the mixture of stabilizers, it was possible to tune the growth of NPs and thus to obtain larger nanoparticles exhibiting interesting magnetic properties; in this case, a segregation of phases cannot be excluded. Watanabe et al. prepared FePd NPs in a similar way; the as-prepared FePd NPs were isolated by centrifugation; the XRD analysis showed a disordered fcc (facecentered cubic) structure. Then, these FePd NPs were treated at $600{ }^{\circ} \mathrm{C}$ under vacuum, leading to a structural change, from fcc to $\mathrm{L} 1_{0}$-ordered fct (face-centered tetragonal) structure after the annealing process. ${ }^{116} \mathrm{L1}_{0}$ FePd NPs exhibited a higher coercive force than fcc FePd NPs isolated after reaction (2040 Oe and 630 Oe, respectively). This change of structure has been also observed when FePd NPs prepared by a polyol process were further transformed in bulk magnets by spark plasma sintering. ${ }^{117}$ The chemical composition of FePd NPs can strongly impact on the magnetic properties. It has been proved that the presence of interstitial carbon atoms on FePd NPs, coming from the co-solvent used (ether derivatives, such as dioctylether and diphenylether), prevents the structural change from disordered fcc to $\mathrm{L}_{0}$-ordered fct: ${ }^{117}$ high content of interstitial carbon hinders the diffusion of Fe and Pd atoms, and then the structural 
change is prevented. Lezama and co-workers have also studied the impact of the chemical synthesis on the magnetic properties of FePd NPs. ${ }^{118}$ They prepared FePd NPs using benzyl ether or toluene as solvent and 1,2-hexadecanediol as reducing agent, in the presence of a mixture of stabilizers (oleic acid and oleylamine, sometimes also adding dodecanethiol). Depending on the relative $\mathrm{Fe} / \mathrm{Pd}$ ratio of precursors, different compositions were obtained $\left[\mathrm{FePd}_{3.4}(\mathrm{~A}), \mathrm{FePd}_{3.2}(\mathrm{~B})\right.$, $\mathrm{FePd}_{5}(\mathrm{C}), \mathrm{FePd}_{1.4}(\mathrm{D}), \mathrm{FePd}_{4}(\mathrm{E})$, and $\left.\mathrm{FePd}_{1.6}(\mathrm{~F})\right]$, showing spherical nanoparticles from 5.0 to $8.3 \mathrm{~nm}$ (TEM data). Magnetization measurements evidenced a superparamagnetic behavior in agreement with the absence of hysteresis at room temperature (Figure 16); from electron magnetic resonance data, it is observed that for higher iron contents (more than 16\%) the plausible Pd magnetic polarization is practically inexistent. Zero-valent iron state is highly sensitive to oxidation, and FePd NPs can be easily oxidized. Actually, FePd(core)-iron oxide(shell) NPs have been obtained, showing enhanced magnetic properties and increased thermal and chemical stability of NPs in relation to oxide-free FePd nanomaterials. ${ }^{119}$
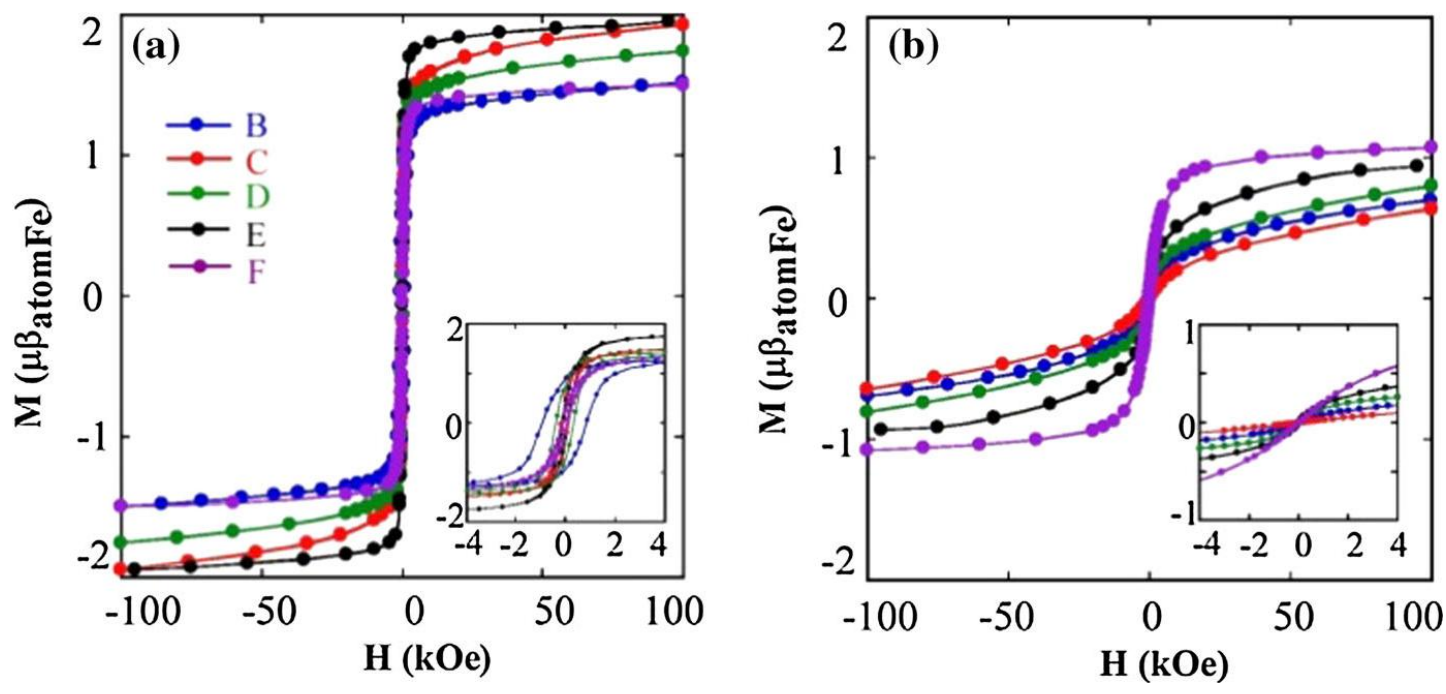
Figure 16. Magnetization vs magnetic field curves measured at $5 \mathrm{~K}$ (a) and room temperature (b) for FePd NPs of different compositions: $\mathrm{FePd}_{3.2}(\mathrm{~B}), \mathrm{FePd}_{5}(\mathrm{C}), \mathrm{FePd}_{1.4}(\mathrm{D}), \mathrm{FePd}_{4}(\mathrm{E})$, and $\mathrm{FePd}_{1.6}(\mathrm{~F})$. Reprinted with permission from Springer Nature, ${ }^{118}$ license number 4558861174833.

\section{Cobalt}

$\operatorname{Pd}($ core $) C o($ shell $)$ nanoparticles with different compositions $\left(\mathrm{Pd}_{1} \mathrm{Co}_{2}, \mathrm{Pd}_{1} \mathrm{Co}_{1}, \mathrm{Pd}_{2} \mathrm{Co}_{1}\right)$ were prepared by a sequential methodology, starting by the reduction of $\mathrm{Pd}(\mathrm{acac})_{2}$ in $\mathrm{EG}$ in the presence of PVP at $120^{\circ} \mathrm{C}$; after $30 \mathrm{~min}, \mathrm{Co}(\mathrm{acac})_{2}$ was added and the temperature increased at $180{ }^{\circ} \mathrm{C}$ with a rapid injection of $\mathrm{NaOH}$ for accelerating the reduction of $\mathrm{Co}$ (II) ions. ${ }^{120}$ The former PdNPs showing a high density of states helps to reduce $\mathrm{Co}(\mathrm{II})$ to $\mathrm{Co}(0)$. PXRD analysis mainly showed the presence of $\mathrm{Pd}$ fcc and Co fcc structures, together with $\mathrm{Co}(\mathrm{OH})_{2}$ phase. TEM combined with EDS analyses evidenced the formation of spherical NPs of 7-10 nm of mean diameter (4-6 nm for inner core and 2-6 nm corresponding to outer shell thickness) with the same composition than that used in the synthesis of these BMNPs.

\section{Nickel}

In 1999, Toshima and co-workers reported the synthesis of NiPd BMNPs by co-reduction of $\mathrm{Pd}(\mathrm{OAc})_{2}$ and $\mathrm{NiSO}_{4}$ salts in $\mathrm{EG}$ in the presence of PVP at $198{ }^{\circ} \mathrm{C}$ under basic conditions. ${ }^{121}$ The resulting colloidal solutions were stable for months, without any sign of agglomeration probably due to the efficient stabilization of PVP and keeping the BMNPs surface state thanks to the reductive medium (EG). They obtained BMNPs over an entire composition range in agreement with the continuous fcc solid solution formed by the bulk metals. ${ }^{86}$ TEM analyses showed the formation of well-dispersed small spherical BMNPs (from 1.5 to $2.3 \mathrm{~nm}$, with standard deviations in the range $0.27-0.37 \mathrm{~nm}$ ) and HRTEM analyses pointed to the formation of 
nanoalloys (determination of the cubic parameter). PXRD, XPS, XANES, and EXAFS analyses proved both metals are in the zero-valence state and that they formed random alloys. Based on this work, Yao and co-workers have prepared NiPd nanoalloys studying in particular their magnetic properties. ${ }^{122}$ For nickel rich BMNPs, both the saturation magnetization (Ms) and coercive force (Hc) increased: for Ni/Pd molar ratio from 3:7 to 7:3, Ms varied from 7.8 to 41.3 $\mathrm{emu} / \mathrm{g}$ and $\mathrm{Hc}$ from 1.2 to $2.2 \mathrm{kOe}$. The high heat-generation capabilities of these nanoalloys offer alternative means to conventional heating, thanks to the high specific power absorption rates.

Kuhn and co-workers have prepared shape-controlled NiPd BMNPs, leading to the formation of nanocubes and nanorods by an adapted polyol methodology. ${ }^{123}$ NiPd nano-objects were prepared by simultaneous reduction of metal precursors (i.e. $\mathrm{Ni}(\mathrm{acac})_{2}$ and $\left.\left(\mathrm{NH}_{4}\right)_{2}\left[\mathrm{PdCl}_{4}\right]\right)$ in 1,5pentanediol in the presence of PVP and trimethyl(tetradecyl)ammonium bromide (TTAB) as capping agents, at different temperatures (in the range 140-160 ${ }^{\circ} \mathrm{C}$ ). Depending on the temperature and TTAB/total metal molar ratio, TEM analyses evidenced the formation of different shape compositions (F). The optimized conditions to mainly obtain nanocubes and nanorods was at $160{ }^{\circ} \mathrm{C} \mathrm{TTAB} /$ total metal molar ratio of $30 / 1$. At high temperature $\left(180{ }^{\circ} \mathrm{C}\right)$ and high TTAB/total metal molar ratio (50/1), no control of the shapes was observed. PXRD analyses showed the presence of $\mathrm{NiO}$ phase. STEM-EDS analyses proved the presence of both metals in the anisotropic BMNPs randomly distributed. As observed in previous works, ${ }^{41}$ the presence of bromide anions was able to favor the reduction of metal salts, the use of a milder reducing agent and relative low temperatures permit to privilege the growth of NPs over some facets; in this work, for TTAB/total metal molar ratio lower than 50/1, (100) and (110) facets were favored, thus leading to cubic and rod-like bimetallic nanoparticles (Figure 17). 

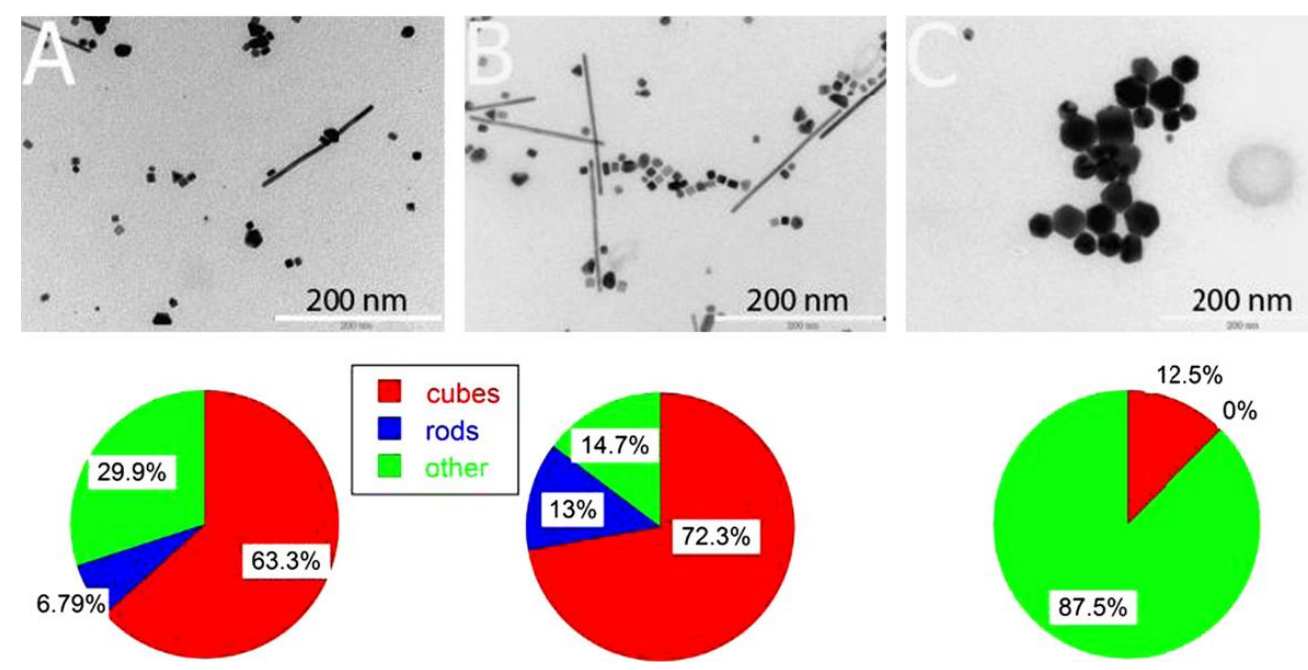

Figure 17. TEM images for NiPd BMNPs with the corresponding shape distribution graphs as a function of TTAB-to-precursor molar ratio at $160{ }^{\circ} \mathrm{C}$ : (A) 15:1, (B) 30:1, and (C) 50:1. Reprinted with permission from Elsevier, ${ }^{123}$ license number 4558861450772.

\section{Copper}

Toshima's group described for the first time the synthesis of CuPd nanoalloys by reduction of the bimetallic hydroxide colloids generated in situ from palladium acetate and copper sulfate under basic conditions in EG and in the presence of PVP as stabilizer; further thermal treatment $\left(198{ }^{\circ} \mathrm{C}\right.$ for $3 \mathrm{~h}$ ) led to the formation of CuPd BMNPs. ${ }^{124}$ Different $\mathrm{Cu} / \mathrm{Pd}$ molar ratios afforded well-defined nanoclusters ( $\mathrm{Cu} / \mathrm{Pd}$ molar ratios from $4 / 1$ to $1 / 2)$. XPS and PXRD analyses proved the zero-valent nature of both metals in the as-prepared nanoalloys. In addition, for copperenriched $\mathrm{BMNPs}$, i.e. $\mathrm{Cu}_{4} \mathrm{Pd}_{1}$, the analysis of $\mathrm{Cu} \mathrm{K}$ edge XANES confirmed the zero valence state of copper, corroborated by EXAFS. ${ }^{125}$ These latter BMNPs showed reproducible SERS spectra, which gave data about the nature of adsorbed organic molecules; the alloying of $\mathrm{Cu}$ with $\mathrm{Pd}$ made $\mathrm{Cu}$ atoms electro-deficient, favoring the adsorption of anions (carboxylates, thiols). These results evidence that the presence of the stabilizer PVP did not hinder the interaction of 
substrates on the metal surface, which is a crucial aspect for catalytic applications. Recently, Shen and co-workers have prepared $\mathrm{Pd}_{\mathrm{x}} \mathrm{Cu}_{100-\mathrm{x}}$ nanoalloys $(\mathrm{x}=20,40,60,80)$ of sizes in the range of 7-9 $\mathrm{nm}$ in EG and using oleylamine as capping agent. In contrast to previous reports, the CuPd BMNPs were treated at 2 different temperatures: in a first stage, the mixture containing all the reagents was heated at $120{ }^{\circ} \mathrm{C}$ for exclusively reducing $\mathrm{Pd}(\mathrm{II})$ to $\mathrm{Pd}(0)$ leading to the formation of Pd clusters; then the mixture was heated at $200{ }^{\circ} \mathrm{C}$ in order to fully reduced both metals. At these temperatures, the diffusion of $\mathrm{Pd}$ and $\mathrm{Cu}$ nuclei was favored, permitting the sizecontrolled synthesis of nanoalloys. STEM-EDS line scanning analyses evidenced the formation of alloys, together with PXRD data. $\mathrm{Pd}_{80} \mathrm{Cu}_{20}$ BMNPs catalyzed the nitrobenzene hydrogenation by a synergy effect: $\mathrm{Pd}$ atoms activate hydrogen and $\mathrm{Cu}$ atoms promoted the adsorption of nitrobenzene.

In our group, we have recently reported the synthesis of $\mathrm{PdCu}$ BMNPs prepared by co-reduction of $\mathrm{Pd}(\mathrm{OAc})_{2}$ and $[\mathrm{Cu}(\mathrm{TMEDA})(\mu-\mathrm{OH})]_{2} \mathrm{Cl}_{2}$ (TMEDA = tetramethylethylendiamine) in neat glycerol under hydrogen pressure $\left(3\right.$ bar) at $120{ }^{\circ} \mathrm{C}$ in the presence of PVP. ${ }^{126}$ Under optimized conditions, small (mean diameters of 2.8 and $3.8 \mathrm{~nm}$ for $\mathrm{Pd} / \mathrm{Cu}$ molar ratios of $1 / 2$ and $1 / 1$ respectively), spherical and well-dispersed PdCu BMNPs were obtained (Figure 18). The asprepared BMNPs were characterized by (HR)TEM and XPS both in glycerol solution (taking advantage of the negligible vapor pressure of glycerol) and solid state (isolated by centrifugation). Both metals showed zero-valent state as proved by XPS and cyclic voltammetry analyses. Depending on the $\mathrm{Pd} / \mathrm{Cu}$ molar ratio, different types of structures were observed: for $\mathrm{Pd} / \mathrm{Cu}$ ratio of $1 / 1$, cluster-in-cluster arrangement; for $\mathrm{Pd} / \mathrm{Cu}$ ratio of $1 / 2$, random alloy; and for $\mathrm{Pd} / \mathrm{Cu}$ ratio of $2 / 1$, a mixture of monometallic nanoparticles as proven by HADDF-STEM-EDS 
analyses. $\mathrm{Pd}_{9} \mathrm{Cu}_{10}$ NPs found interesting applications in semi-hydrogenation of alkynes (see below section 3).

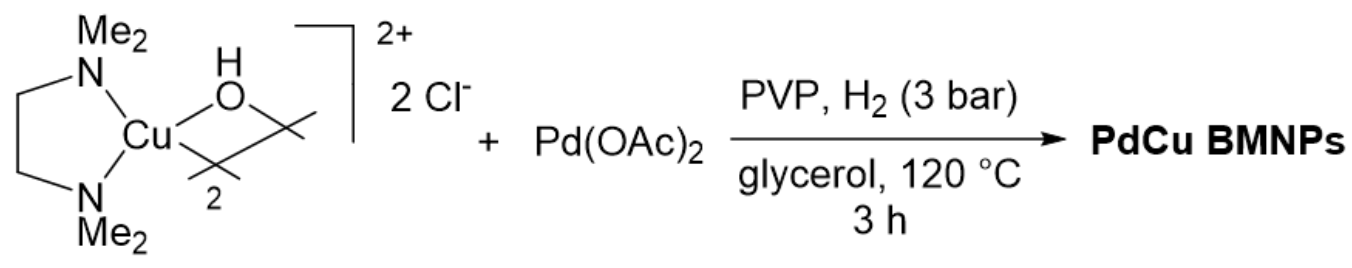

$$
\begin{aligned}
& \mathrm{Pd} / \mathrm{Cu} \text { molar ratio }=1 / 2,1 / 1,2 / 1 \\
& \text { Monomer PVP } / \mathrm{Cu} / \mathrm{Pd}=40 / 1 / 1
\end{aligned}
$$
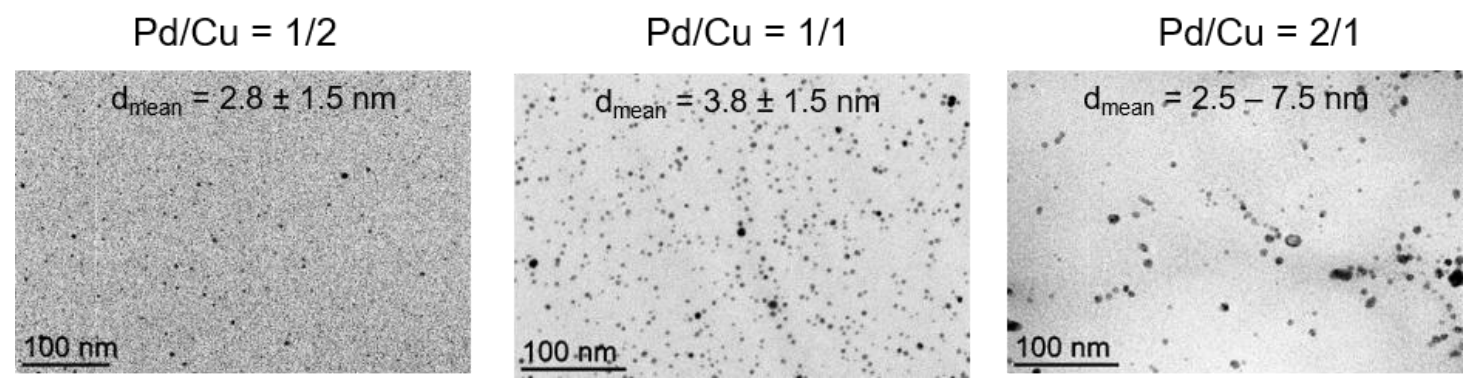

Figure 18. Synthesis of $\mathrm{PdCu}$ BMNPs in neat glycerol from $\mathrm{Pd}(\mathrm{II})$ and $\mathrm{Cu}(\mathrm{II})$ precursors under molecular hydrogen pressure using PVP as stabilizer (top). TEM images recorded in glycerol for each of the three systems (bottom). ${ }^{126}$

For a summary of polymetallic Pd-based NPs synthetic conditions and characterization outcomes, see Table S2 in the Supporting Information.

\subsection{Hydroxyl compounds from natural sources}

The synthesis of metal nanoparticles by chemical bottom-up methodologies involves multiparameter protocols; the kind of metal precursor (salts or well-defined organometallic compounds), solvent, reducing agent and stabilizer are particularly crucial in order to produce nanomaterials with well-defined morphology, structure and surface state, features undoubtedly related to their distinctive physicochemical properties. ${ }^{9,12}$ In the last years, a special emphasis has 
been devoted to develop environmentally friendly procedures, mainly using polyfunctional reagents coming from natural sources. ${ }^{127-129}$ In the biogenic synthesis of metal nanoparticles, the use of extracts from plants has experienced a significant growth due to the chemical reduction properties shown by polyphenols, alkaloids, polysaccharides and terpenoids among others, commonly present in their composition; they can also act as stabilizers using in general water as solvent. ${ }^{12}$ Most of the reported methods consist in the direct use of extracts from different plants coming from diverse parts (leaves, roots, flowers, fruits), but often without identification of the molecules responsible of the nanoparticles formation. ${ }^{12}$ These works were initially focused on silver and gold, ${ }^{130,131}$ but in the last ten years some contributions concerning palladium, both mono and bimetallic Pd-based nanoparticles and supported counterparts has been published. ${ }^{132,133}$ In the present review, we highlight the contributions focusing on characterization and PdNPs formation mechanisms using plant extracts.

One of the first reports concerning the synthesis of PdNPs using natural extracts was reported by Varma and co-workers in 2008, using coffee and tea extracts. ${ }^{134}$ For the different brands of tea and coffee used, PdNPs were formed in aqueous solution from $\mathrm{PdCl}_{2}$ in the presence of the corresponding natural extract at room temperature, obtaining spherical particles from $c a .5$ to 40 $\mathrm{nm}$ (Figure 19). The extracts are mainly constituted by caffeine and polyphenols. The reduction potential of caffeine is $0.3 \mathrm{~V} v s \mathrm{SCE}$ which is enough to reduce $\mathrm{Pd}(\mathrm{II})$ salts (Pd(II) reduction potential is $0.915 \mathrm{~V} v \mathrm{SCE}$ ); polyphenols and oxidized caffeine are proposed as capping agents avoiding the agglomeration of particles. 

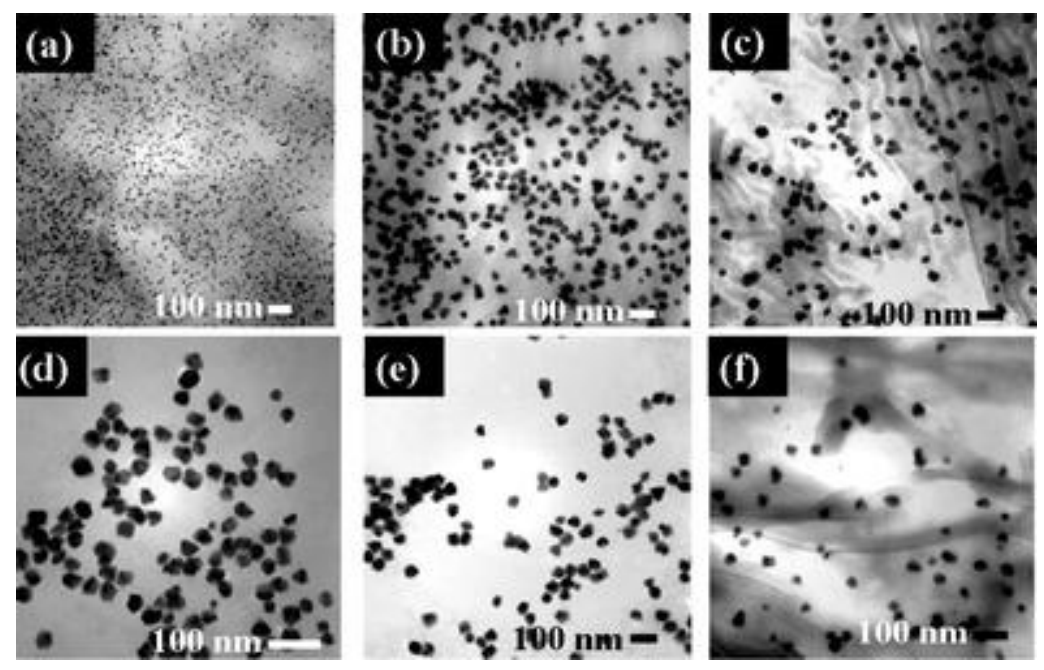

Figure 19. TEM images of PdNPs synthesized using (a) Sanka coffee, (b) Bigelow tea, (c) Luzianne tea, (d) Starbucks coffee, (e) Folgers coffee, and (f) Lipton tea extract. Reprinted with permission from Royal Society of Chemistry, ${ }^{134}$ license number 4558870466147.

Analogously, other authors have applied a similar procedure using other types of extracts. Therefore, Philip and co-workers prepared spherical and small nanoparticles (mean size in the range 2.5-4.5 $\mathrm{nm}$ ) from extracts of Anacardium occidentale leaves; authors stated that the water soluble polyols act as reducing agents of $\mathrm{Pd}(\mathrm{II})$ ions. ${ }^{135} \mathrm{Li}$ and co-workers also obtained regular and small spherical nanoparticles (mean size in the range 3.2-6.0 depending on $\operatorname{Pd}(\mathrm{II})$ ions concentration) using Cinnamomum camphora extract leaves, proving that polyols are the responsible of the metallic ions, favoring the stabilization of PdNPs. ${ }^{136}$ Bigger nanoparticles (mean size ca. 20-25 nm) were obtained from Pulicaria glutinosa extracts; ${ }^{137}$ authors proved that the plant extract not only acts as bioreducing agent but also as capping agent as evidenced by FTIR spectroscopy. More recently, Kim and co-workers have reported the synthesis of PdNPs using fruit juices, leading to spherical PdNPs exhibiting uniform size distributions (between 2-25 nm depending on the juice type); ${ }^{138}$ they have proved that sugars and polyols present in the juices are in a first time reducing agents, and inhibiting the agglomeration of nuclei in a last stage; the 
as-prepared PdNPs showed size-dependent SERS activities with applications in ultrasensitive detection of organic molecules. Cynarine, a hydroxycinnamic acid extracted form artichoke leaves, ${ }^{139}$ Charanthus roseus leaf extract where eight metabolites were identified by GC-MS, ${ }^{140}$ and Terminalia chebula aqueous extract where polyphenols act as reducing agents and their oxidized forms as stabilizers ${ }^{141}$ led to the formation of bigger PdNPs (ca. from 25 to $100 \mathrm{~nm}$ ) in the presence of anisotropic particles. ${ }^{141}$ It is important to highlight that the biosynthesis of metalbased nanoparticles is also an efficient approach to prepare bimetallic nanoparticles. In this frame, $\mathrm{Li}$ and co-workers have reported the preparation of alloyed $\mathrm{PdAu}$ nanoparticles, synthesized from Cacumen platycladi leaf extract, exhibiting a mean size of $7.4 \mathrm{~nm} .{ }^{142}$

\section{Pd-based nanocatalysts applied in hydrogenations}

Organic synthesis involving the activation of small nonreactive molecules, such as $\mathrm{H}_{2}, \mathrm{~N}_{2}, \mathrm{NH}_{3}$, $\mathrm{H}_{2} \mathrm{O}$, and $\mathrm{CO}_{2}$ by transition metal catalysts or by enzymes represents an attractive approach for industrial purposes in terms of high atom-efficiency. As an example, catalytic hydrogenation remains the main reaction for the reduction of unsaturated bonds. ${ }^{143-146}$ Current researches in this field tackle the improvement of $\mathrm{H}_{2}$ production processes, together with the enhancement of catalyst performance and reaction medium optimization towards greener and safer hydrogenation protocols.

To override both the dependence on fossil fuels and diminish $\mathrm{CO}_{2}$ emissions associated to classical $\mathrm{H}_{2}$ production processes, alternatives consisting in its generation from $\mathrm{NH}_{3},{ }^{147}$ biomass resources, ${ }^{148}$ water splitting ${ }^{149}$ or hydrogen transfer reactions are actively sought. ${ }^{150}$

Concerning the choice of solvent used for hydrogenation applications, alternatives to classical organic solvents are actively pursued due to the risks associated to their high flammability, 
toxicity and volatility properties. ${ }^{151}$ The solvent plays key roles in hydrogenation reactions: First, due to the interactions with the substrate (polarity, solubility, mass transfer) ${ }^{152-154}$ or/and with the catalyst ${ }^{155,156}$ but also due to the solubility of $\mathrm{H}_{2}$ gas in the medium. ${ }^{157}$ The $\mathrm{H}_{2}$ solubility in alcohol clearly increases with temperature and pressure and also with the number of carbon atoms; the solubility of $\mathrm{H}_{2}$ is higher in mono-alcohols than in diols (e.g. Henry's constant diminishes from 20000 in 1,2-ethanediol to $4000 \mathrm{bar}^{-1}$ in ethanol at $\left.50{ }^{\circ} \mathrm{C}\right) .{ }^{158} \mathrm{The}_{2}$ solubility follows a similar trend. ${ }^{159}$

The quest for hydrogenation catalysts exhibiting enhanced selectivity and reactivity is an active research field for $\mathrm{Pd}, \mathrm{Ru}, \mathrm{Rh}$, and Ir transition metal complexes. ${ }^{160}$ Nanocatalysis in particular offers privileged means towards hydrogenation processes, given the extended surface area ratios. ${ }^{161,162} \mathrm{Pd}$ NPs, for instance, show high reactivity towards $\mathrm{H}_{2}$ activation ${ }^{163-166}$ as palladium is known to adsorb up to 900 times its own volume. ${ }^{167,168}$ Thus, the (chemo)-selectivity of these nanomaterials towards hydrogenation is highly controlled by surface interactions with the modifiers/stabilizers, the substrates or even the supports. ${ }^{169,170}$

In this section, we describe the use of palladium nanoparticles as hydrogenation catalysts in polyols. A few reports concerning bimetallic Pd based NPs have been described for hydrogenations in EG. In this context, bimetallic PdNi NPs were active in the hydrogenation of nitrobenzene under smooth conditions $\left(30{ }^{\circ} \mathrm{C}\right.$ and atmospheric pressure of $\left.\mathrm{H}_{2}\right) .{ }^{121}$ Moreover, PdCo NPs were efficient catalysts for both hydrogenation and oxidation of $\mathrm{C}=\mathrm{C}$ bonds. Nearambient pressure XPS spectra (NAPXPS) revealed that $\mathrm{Co}(0)$ and $\mathrm{Co}(\mathrm{III})$ sites are the responsible of the hydrogenation and oxidation of styrene, respectively; this dual catalytic behavior was attributed to the intrinsic electronic modifications between the two metals during 
the catalytic processes. ${ }^{120}$ Hitherto, mostly hydrogenations conducted in PEG and glycerol have been reported in the literature.

\subsection{Hydrogenation reactions catalyzed by PdNPs in PEG}

Polyethylene glycols (PEG) are interesting for catalysis because they are soluble in polar and non-polar solvents and insoluble in aliphatic hydrocarbons. ${ }^{171}$ They are inexpensive and less hazardous than other organic solvents. Additionally, PEG are stable under ambient conditions due to their negligible vapor pressure and do not release volatile organic compounds. The applications of PEG in hydrogenations involve three aspects: i) phase transfer catalysts, ${ }^{172}$ ii) supported catalysts, ${ }^{173}$ and iii) solvents. ${ }^{174,175}$ In relation to the solubility of $\mathrm{H}_{2}$, for example for PEG400 is higher compared to an ionic liquid such as 1-ethyl-3-methylimidazolium tetrafluoroborate. ${ }^{176}$

Based on the work of Zhang and coworkers, ${ }^{177}$ PdNPs were prepared in PEG from decomposition of $\mathrm{Pd}(\mathrm{OAc})_{2}$ by polyol methodology at $90{ }^{\circ} \mathrm{C}$ for $2 \mathrm{~h} .{ }^{178}$ It was observed that for a same concentration in precursor and the same mass of PEG, the size of the PdNPs increases with the molecular weight of polymer (PdNPs of 3-5 nm mean diameter for PEG800 and PEG1000; 7-9 $\mathrm{nm}$ for PEG2000 and 19-23 $\mathrm{nm}$ for PEG4000). These as-prepared PdNPs were tested for the hydrogenation of cyclohexene as benchmark reaction (with a substrate/Pd ratio of 5500:1 at $70^{\circ} \mathrm{C}$ ). With PEG4000 the viscosity of the polymer is too high triggering a low conversion of cyclohexene; PdNPs obtained in this solvent were larger than the previously described under similar conditions ${ }^{177,179}$ Among these catalysts, PdNPs stabilized by PEG2000 showed the highest activity and was used to run the hydrogenations of differently substituted alkenes 
(Scheme 1). The authors explained that small particles are not the most active due to the smaller distance between the polymer and the metallic surface which disfavors the mass transfer. These catalysts are highly active; with PdPEG1000-NPs and PdPEG2000-NPs, 60 and 55 min respectively were needed to reach full conversion of cyclohexene, in comparison to $\mathrm{Pd} / \mathrm{C}$ which required higher pressure of $\mathrm{H}_{2}$ (40 bar instead of 10 bar for PEG1000 and PEG2000).

Scheme 1. Pd-catalyzed hydrogenation of different alkenes catalyzed by PdNPs in PEG2000. ${ }^{178}$

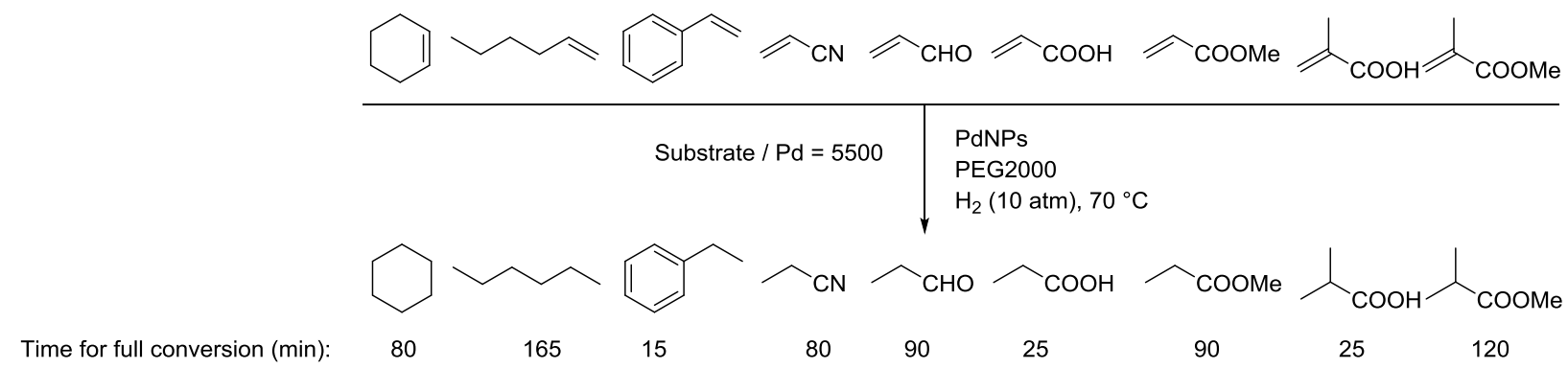

In the field of biodiesel development, there is an increasing interest in Z-mono-unsaturated compounds instead of poly-unsaturated ones while avoiding side $Z / E$ olefin isomerization in order to improve the cold-flow properties as fuels. In this context, Liu et al. described the use of PdNPs in PEG with different molecular weights $(\mathrm{Mw}=400,600,1000,2000$, and 4000) as catalysts in order to reduce methyl linoleate into (Z)-methyl oleate while minimizing isomerization towards $(E)$-methyl elaidate. ${ }^{179}$ The best catalyst was prepared from $\mathrm{Pd}(\mathrm{OAc})_{2}$ in PEG4000, at $75^{\circ} \mathrm{C}$ for $2 \mathrm{~h}$, which solidified upon cooling down to room temperature, leading to 
PdNPs with a mean diameter size of $4.0 \mathrm{~nm}$. As observed by UV-vis during the formation of the PdNPs using PEGs of different molecular weights, high Mw PEGs are better reducing agents; low molecular weights of PEGs can stabilize better Pd(II) complexes hindering the reduction to $\operatorname{Pd}(0)$. Upon optimization of the experimental parameters, the best conditions were the ones shown in Scheme 2. Despite that substrate hydrogenation was faster with PEG-400 and 1000, the yield of methyl oleate was better when PEG-600, 2000, and 4000 were used. PEG2000 and PEG4000 were finally chosen for this study due to the instability of PdNPs generated in PEG600 by formation of a precipitate.

Scheme 2. Pd-catalyzed hydrogenation of different alkenes catalyzed by PdPEG4000-NPs. ${ }^{179}$

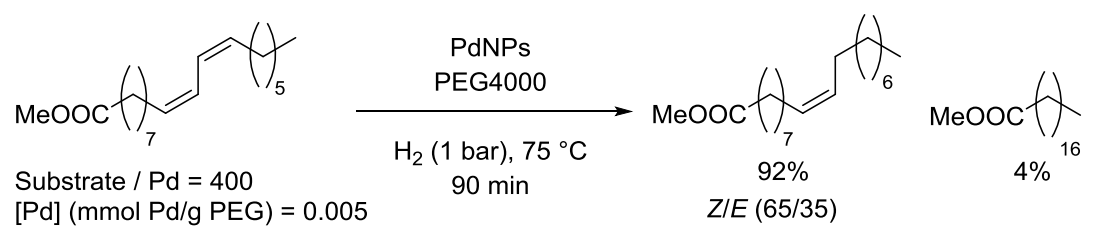

Later on, the same authors have adapted the experimental conditions to the partial hydrogenation of sunflower oil derived fatty acid methyl esters (FAME). ${ }^{180}$ The best conditions were the following ones: FAME/Pd $=2000,80{ }^{\circ} \mathrm{C}, 20 \mathrm{bar}_{2}$, and $1500 \mathrm{rpm}$ stirring speed. This methodology permitted to obtain full conversion of methyl linoleate with a high selectivity towards methyl oleate (85\%), while minimizing the total reduction towards stearic acid (only $7 \%$ ). This catalyst was reused up to 5 times without loss of activity nor selectivity. 
The Wang group developed a thermoregulated PEG biphasic system, where PEG was used as stabilizer of PdNPs and also as a catalytic biphasic system with a toluene/heptane mixture. When the solution was heated at $70{ }^{\circ} \mathrm{C}$, the system became monophasic and when cooled, the organic compounds could be easily separated from the PEG-Pd catalytic phase. ${ }^{181}$ PdNPs stabilized by PEG4000 were in situ formed in this mixture of solvent of PEG4000/toluene/heptane = 2/3/1 using $\mathrm{Pd}(\mathrm{OAc})_{2}$ as precursor for the hydrogenation of 1,5-cyclooctadiene (1,5-COD). The asprepared PdNPs exhibited a mean diameter size of $3.1 \pm 0.8 \mathrm{~nm}$. The best conditions to get high conversion in 1,5-COD and high selectivity in cyclooctene are presented in Scheme 3.

Scheme 3. Hydrogenation of 1,5-cyclooctadiene catalyzed by PdNPs in PEG4000/toluene/heptane biphasic system. ${ }^{181}$

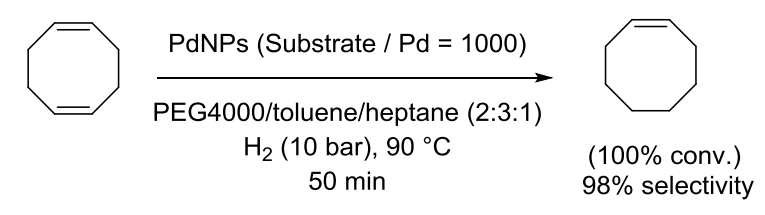

Later, the same authors used hydrated $\mathrm{Na}_{2}\left[\mathrm{PdCl}_{4}\right]$ as metallic precursor for the analogous synthesis of thermoregulated PdNPs in a PEG4000/toluene/heptane biphasic system. The asprepared catalyst featuring nanoparticles of a smaller size $(1.9 \pm 0.5 \mathrm{~nm})$ than the ones obtained previously from $\mathrm{Pd}(\mathrm{OAc})_{2}$ were applied for the hydrogenation of cinnamaldehyde (Scheme 4). ${ }^{182}$ This catalytic biphasic phase was recycled up to eight times without loss of activity; nor PdNPs size changes were evidenced by TEM, nor Pd leaching in the organic fractions, which were analyzed by ICP-AES. 
Scheme 4. Hydrogenation of cinnamaldehyde catalyzed by PdPEG4000/toluene/heptane in biphasic conditions. ${ }^{182}$

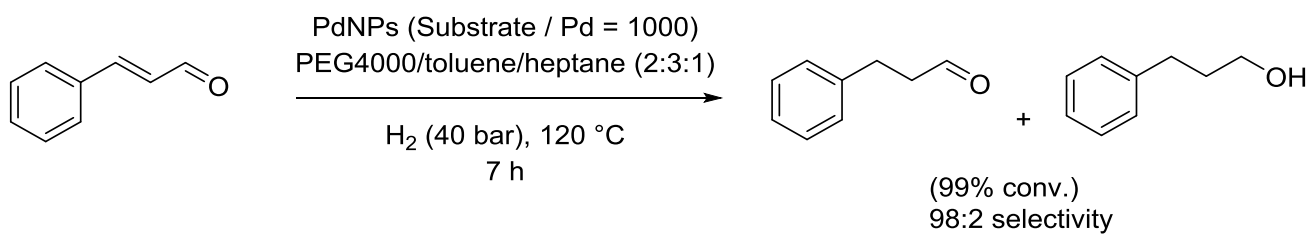

The use of stabilizers in combination with PEG leads to tailor-made PdNPs active nanocatalysts. ${ }^{183}$ Based on a work described by Huang and coworkers in ionic liquids, ${ }^{184}$ phenanthroline was used to stabilize PdNPs in PEG and used as catalyst for the hydrogenation of a series of aliphatic alicyclic and aromatic olefins (Scheme 5). The experimental conditions were optimized by using cyclohexene as substrate and varying different parameters such as temperature, amounts of Pd, PEG and stabilizer, the nature of palladium precursor $\left[\mathrm{Pd}(\mathrm{OAc})_{2}\right.$, $\mathrm{PdCl}_{2}, \mathrm{Pd}(\mathrm{acac})_{2},(\mathrm{acac}=$ acetylacetonate $\left.)\right]$, the nature of the metal $($ acetates of $\mathrm{Cu}, \mathrm{Ni}, \mathrm{Co}$ and $\mathrm{Zn}$ ), and the stabilizer (phenanthroline, PVP, 1-hexadecylaniline). In this study, authors postulated that the molecular weight of PEG had no noticeable influence on the activity of the catalyst, but no experimental data is reported in the paper to support this conclusion. It was proved that phenanthroline prevents the agglomeration of PdNPs (mean size in the range 2-6 nm determined by TEM), giving more active PdNPs catalysts compared to the non-stabilized ones in PEG (mean size in the range $500-1000 \mathrm{~nm}$ determined by TEM); but what is noteworthy to mention is that the recyclability of the catalyst was not affected by the absence of phenanthroline and the activity increased after the first run (84\% conversion of cyclohexene for the first run and $100 \%$ for the second one, maintaining a full conversion up to the sixth run). That point was ascribed by the authors to the fact that the palladium precatalyst was not fully converted after the 
first run towards the formation of nanoclusters. The two other stabilizers (PVP and 1hexadecylaniline) gave the same catalytic behavior than phenanthroline under the same conditions. Notably, the metal leaching promoted by phenanthroline has not been studied (no experimental data is reported); thereby the contribution of homogeneous catalytic regime cannot be excluded.

Scheme 5. Hydrogenation of different alkenes containing aliphatic, alicyclic and aromatic groups catalyzed by phenanthroline-stabilized PdNPs in PEG400. ${ }^{183}$

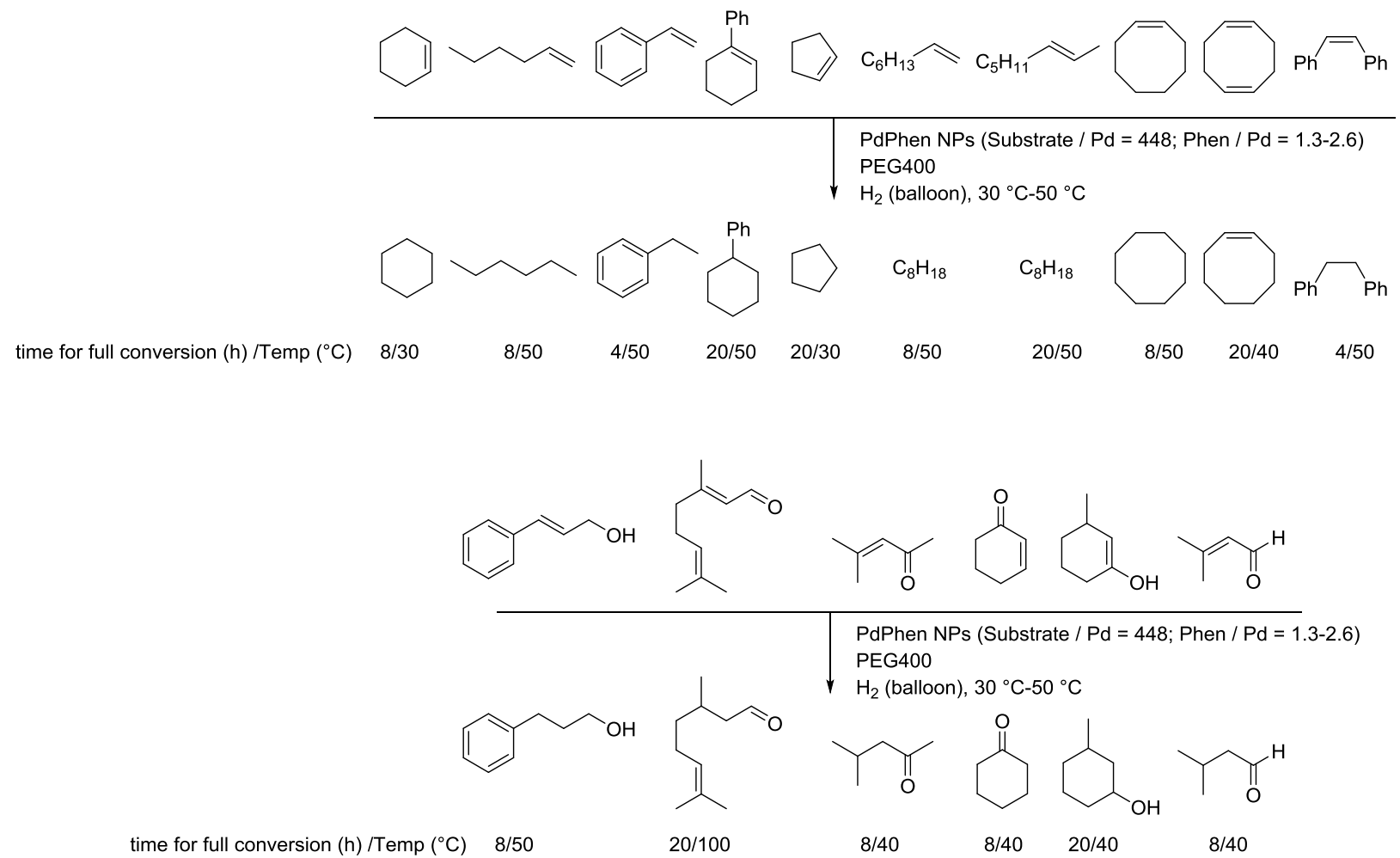

\subsection{Hydrogenation reactions catalyzed by PdNPS in glycerol}


Glycerol presents many advantages, namely, it is nontoxic and biodegradable, it can be produced from renewable sources, and it is able to dissolve many organic compounds. Many organic syntheses have been described thus far in glycerol. ${ }^{185,186}$

In our research team (see section 2.1.2), we developed an efficient catalytic system composed of PdNPs dispersed in neat glycerol and stabilized by TPPTS. ${ }^{74}$ In the absence of TPPTS, PdNPs agglomerated and precipitated in glycerol. These nanoparticles were prepared under $\mathrm{H}_{2}$ in glycerol as solvent, without observing any hydrogenolysis of the solvent. The best active catalytic system was obtained starting from $\mathrm{Pd}(\mathrm{OAc})_{2}$ using 1 equiv. of TPPTS giving nanoparticles with a mean diameter size of $4.1 \pm 1.4 \mathrm{~nm}$. This catalyst was highly active for the hydrogenation of alkenes, alkynes, and nitro compounds (Scheme 6). ${ }^{74}$

Scheme 6. Hydrogenation of styrene derivatives, alkynes and nitro compounds catalyzed by PdTPPTS NPs in glycerol. ${ }^{74}$

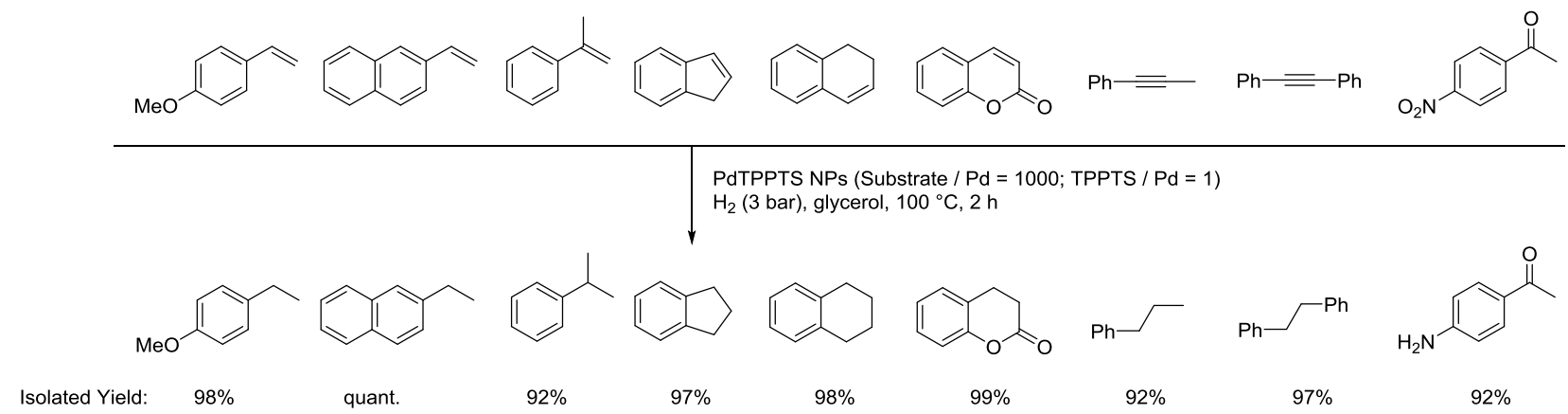

This catalytic colloidal solution of PdTPPTS NPs in glycerol was also applied in sequential coupling/hydrogenation processes (Scheme 7). ${ }^{74}$ 
Scheme 7. Sequential Coupling/hydrogenation processes catalyzed by PdTPPTS NPs in glycerol. ${ }^{74}$

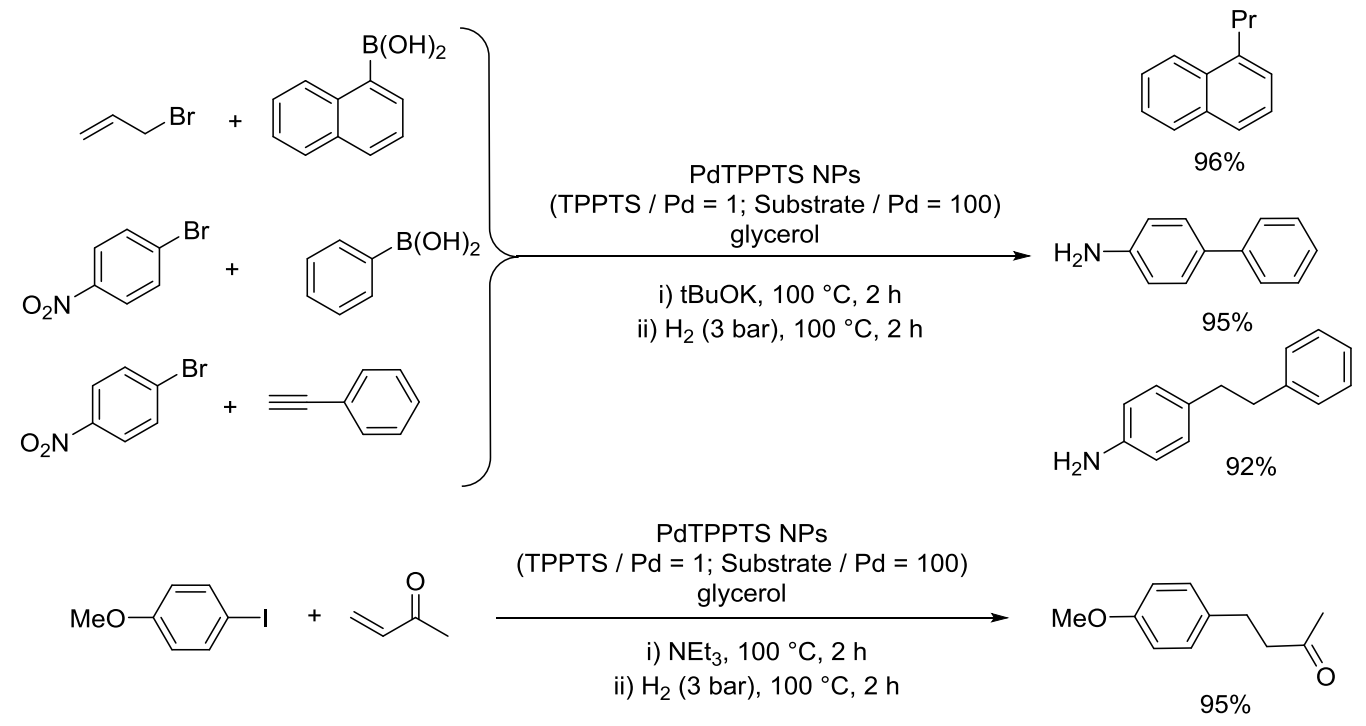

Furthermore, the as-prepared catalytic systems were also active towards coupling/cyclization/hydrogenation tandem reactions for the synthesis of phthalimides, 1-benzyl1,3-dihydroisobenzofuran and 3-(4-substitued-benzyl)isoindoin-1-one derivatives in high yields (>90\%, Scheme 8). ${ }^{187}$ 
Scheme 8. Sequential coupling/cyclisation/hydrogenation processes catalyzed PdTPPTS NPs in glycerol. ${ }^{187}$<smiles>O=C(O)c1ccccc1I</smiles>

$\mathrm{R}_{1}, \mathrm{R}_{2}, \mathrm{R}_{3}=\mathrm{H}$ $\mathrm{R}_{1}=\mathrm{Me}, \mathrm{R}_{2}, \mathrm{R}_{3}=\mathrm{H}$ $\mathrm{R}_{1}=\mathrm{H}, \mathrm{R}_{2}, \mathrm{R}_{3}=\mathrm{Me}$ $\mathrm{R}_{1}, \mathrm{R}_{2}=\mathrm{H}, \mathrm{R}_{3}=\mathrm{Ph}$

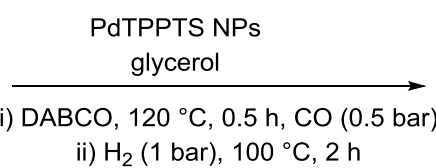

ii) $\mathrm{H}_{2}$ (1 bar), $100^{\circ} \mathrm{C}, 2 \mathrm{~h}$

$$
\text { Substrate } / \mathrm{Pd}=250 \text {, }
$$$$
\text { TPPTS } / \mathrm{Pd}=1
$$<smiles>[R5]Pc1ccccc1CO[OH+]</smiles>
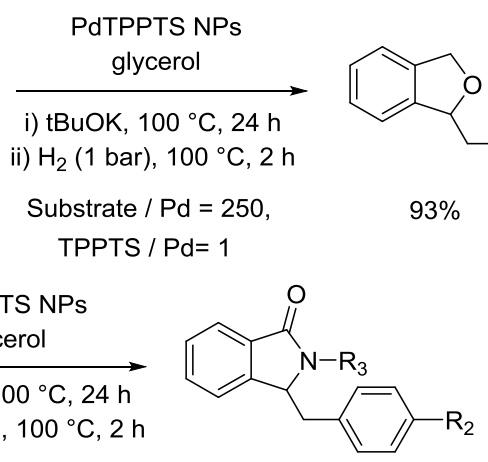

7 examples

$$
\text { 93-98\% }
$$

$\mathrm{R}_{3}=\mathrm{Bu}, \mathrm{Pr}, \mathrm{Cy}$

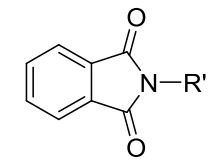

4 examples

94-98\%

$\mathrm{R}^{\prime}$ = propyl, isobutyl, isopentyl, 3-phenylpropyl

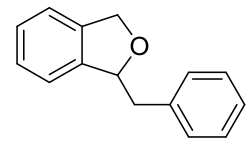
$93 \%$

$$
\mathrm{R}_{3}=\mathrm{Bu}, \mathrm{Pr}, \mathrm{Cy}
$$

An analogous nanocatalyst generated from $\left[\mathrm{PdCl}_{2}(\mathrm{COD})\right]$ as metal precursor and stabilized with benzyl substituted 1,3,5-triaza-7-phosphoadamantane $(\mathrm{PTA}, \mathrm{Pd} / \mathrm{L}$ ratio $=1: 1)$ in glycerol furnished PdNPs with a mean diameter of $3.2 \pm 0.8 \mathrm{~nm} .{ }^{76}$ This catalyst was tested for the 
hydrogenation of E-4-phenylbut-3-en-2-one and was recycled up to ten times without loss of activity (Scheme 9). Notably, the size of the PdPTA NPs was smaller after the tenth run, which could be explained due to an inter-particle reorganization under the catalytic conditions in glycerol.

Scheme 9. Hydrogenation of E-4-phenylbut-3-en-2-one and coumarin catalyzed by PdPTA NPs in glycerol. ${ }^{76}$
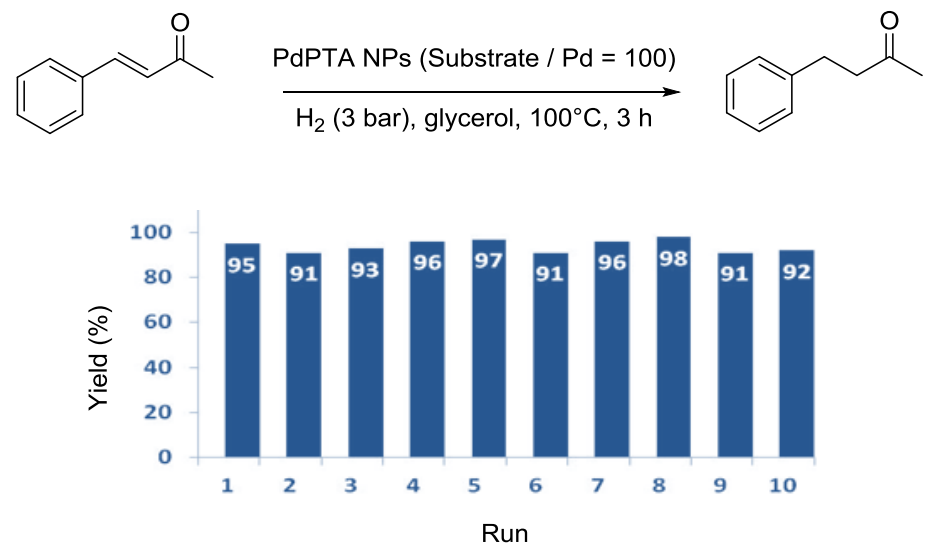

Furthermore, PdPTA nanocatalyst was also applied towards the reduction of 4nitroacetophenone into 4-aminoacetophenone, however further ketone group reduction to 4ethylaniline was also observed (Scheme 10). ${ }^{76}$

Scheme 10. Hydrogenation of 4-nitroacetophenone catalyzed by PdPTA NPs in glycerol. ${ }^{76}$ 


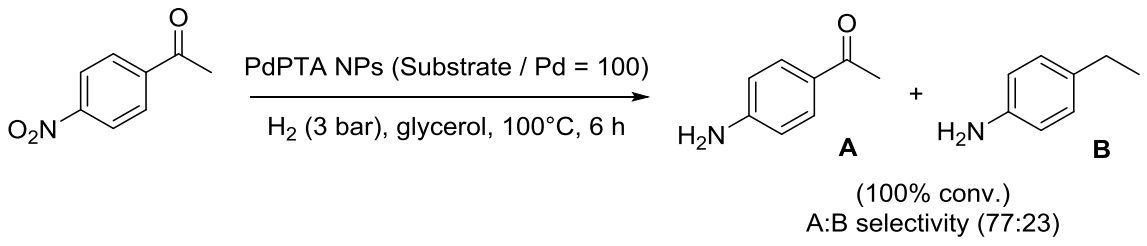

This catalyst was also active in sequential C-C coupling/hydrogenation processes for the synthesis of 4-phenylaniline, affording exclusively this product in 54\% yield (Scheme 11). ${ }^{76}$

Scheme 11. Sequential Coupling/hydrogenation catalyzed by PdPTA NPs in glycerol. ${ }^{76}$<smiles>Nc1ccc(-c2ccccc2)cc1</smiles>

More recently cinchona based alkaloids were also used as PdNPs stabilizers in glycerol. ${ }^{79}$ The best active catalytic system was obtained starting from $\mathrm{Pd}(\mathrm{OAc})_{2}$ (among $\left[\mathrm{PdCl}_{2}(\mathrm{COD})\right]$ and $\left[\mathrm{Pd}_{2}(\mathrm{dba})_{3}\right]$ ) using 1 equiv. of quinidine (among cinchonidine, cinchonine, quinine and quinidine). Quinidine leads to a better dispersion of the particles according to the TEM images with a mean diameter size of $1.4 \pm 0.3 \mathrm{~nm}$. Notably, Pd-cinchona based NPs are more active for the hydrogenation of E-4-phenylbut-3-en-2-one compared to the previously described PdTPPTS NPs (Scheme 12). 
Scheme 12. Hydrogenation of E-4-phenylbut-3-en-2-one catalyzed by PdNPs in glycerol. ${ }^{79}$<smiles>CC(=O)/C=C/c1ccccc1</smiles>

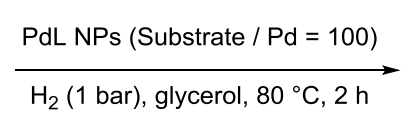

Cinchonidin
Cinchonine

Cinchonine

Quinidine

TPPTS

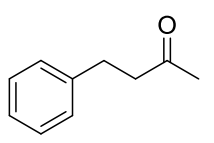

(Conv.) Yield

$(99 \%) \quad 96 \%$

$(79 \%) \quad 74 \%$

$(99 \%) \quad 90 \%$

$\begin{array}{ll}(99 \%) & 98 \% \\ (87 \%) & 85 \%\end{array}$

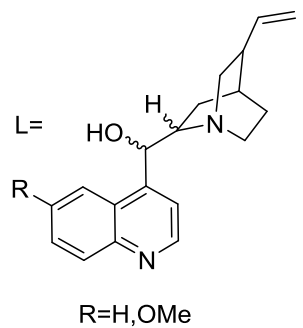

The PdNPs stabilized by quinidine were applied in the hydrogenation reaction of various alkenes and alkynes (Scheme 13). ${ }^{79}$ It is noteworthy to mention that in the case of 1 -dodecene, only $1 \%$ of isomerization product was detected for $99 \%$ conversion into dodecane.

Scheme 13. Hydrogenation of different alkenes and alkynes catalyzed by PdNPs stabilized by quinidine in glycerol. ${ }^{79}$

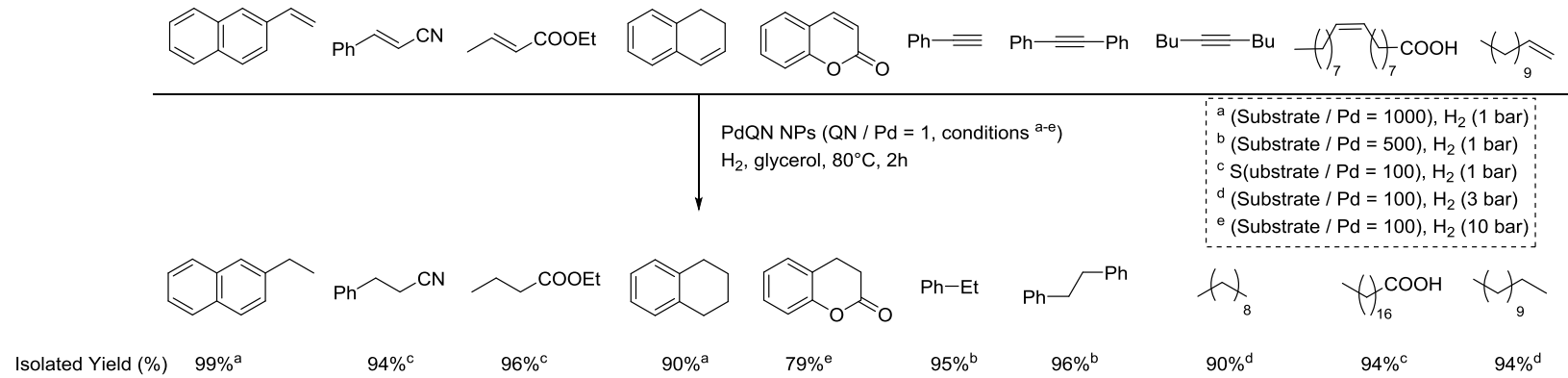

More interestingly the as-prepared PdQN NPs were also applied towards catalytic syntheses of secondary and tertiary amines from carbonyl and amine reagents, as well as in the synthesis of $N$-substituted anilines from nitrobenzene and aldehydes by one-pot reductive amination; in addition, PdQN NPs were efficient catalysts for hydrodebromination reactions (Scheme 14). ${ }^{79}$ 
Scheme 14. Hydrogenation processes catalyzed by PdQN NPs in glycerol. ${ }^{79}$
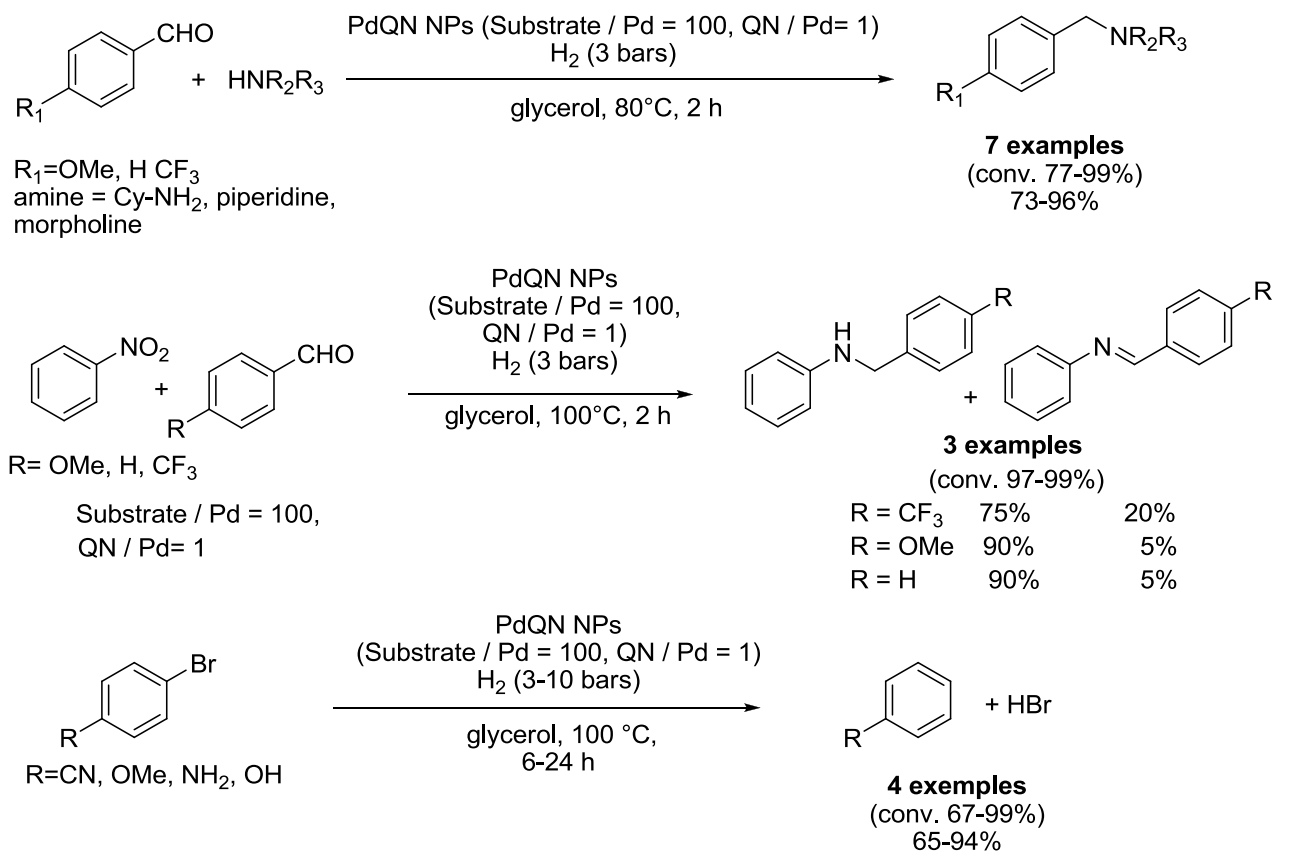

Choline based ionic liquids in neat glycerol were also used as stabilizers for PdNPs in glycerol. ${ }^{188}$ Nanocatalysts were synthesized from $\left[\mathrm{PdCl}_{2}(\mathrm{COD})\right]$ as $\mathrm{Pd}$ precursor and choline $N$ tosylalaninate as stabilizer with a ratio $\mathrm{Pd} / \mathrm{IL}(1: 17)$ under $\mathrm{Ar}$ atmosphere at $80{ }^{\circ} \mathrm{C}$ overnight, leading to nanoparticle mean diameter sizes of $1.7 \pm 0.6 \mathrm{~nm}$. Notably, the water present in the medium triggered the reduction of $\mathrm{Pd}(\mathrm{II})$ to $\mathrm{Pd}(0)$ NPs, as previously observed in the literature. ${ }^{189}$ Contrarily to the PdQN NPs, the hydrogenation of monoalkenes (as 1-dodecene) with PdIL NPs leads to the formation of a high quantity of isomerization products. Although, the hydrogenation was complete and selective for conjugated systems. Thus, the more hindered substrate 1,2,3,4,5-pentamethylcyclopentadiene was selectively reduced into the cyclopentene derivative under 20 bar of $\mathrm{H}_{2}$ pressure (Scheme 15). 
Scheme 15. Hydrogenation of alkenes and alkynes catalyzed by PdNPs stabilized by choline derivative in glycerol. ${ }^{188}$

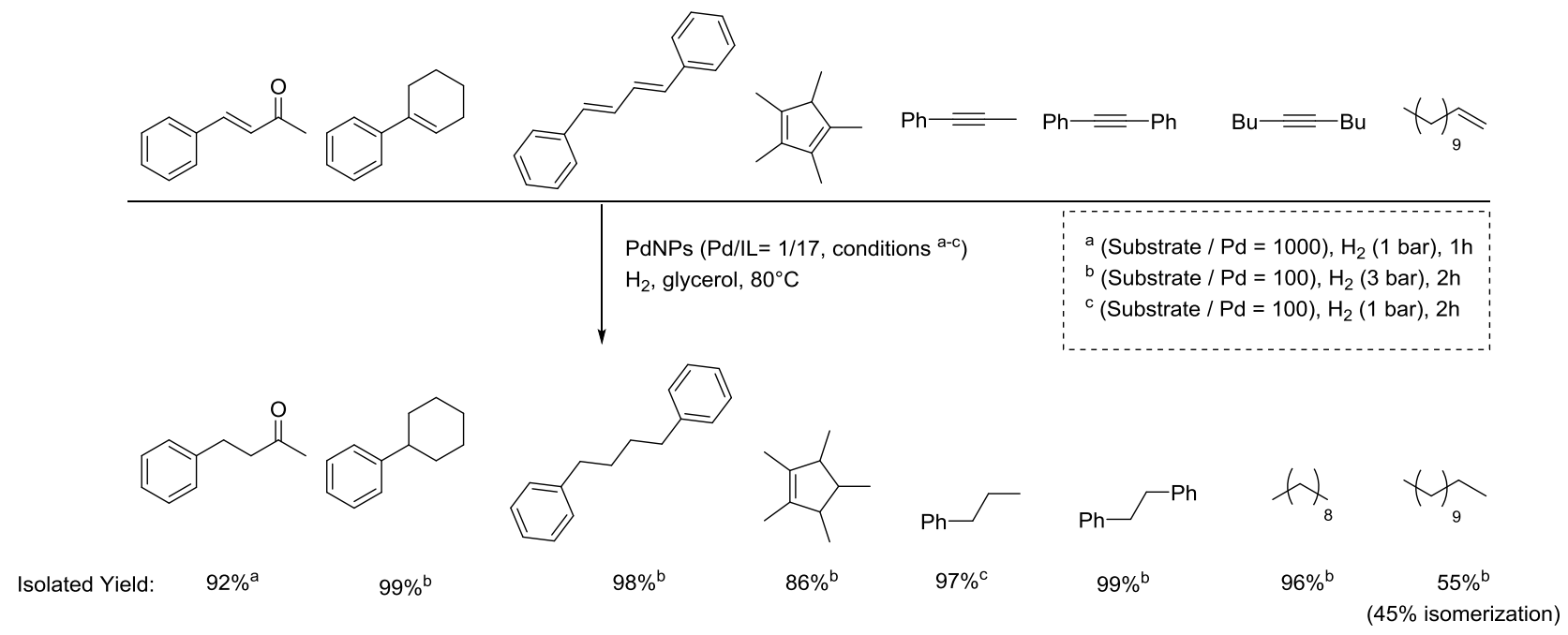

This catalytic system is even more interesting towards the hydrogenation of the carbonyl groups which can be reduced under relatively smooth conditions (Scheme 16). ${ }^{188}$

Scheme 16. Hydrogenation of nitro and carbonyl groups catalyzed by PdIL NPs in glycerol. ${ }^{188}$

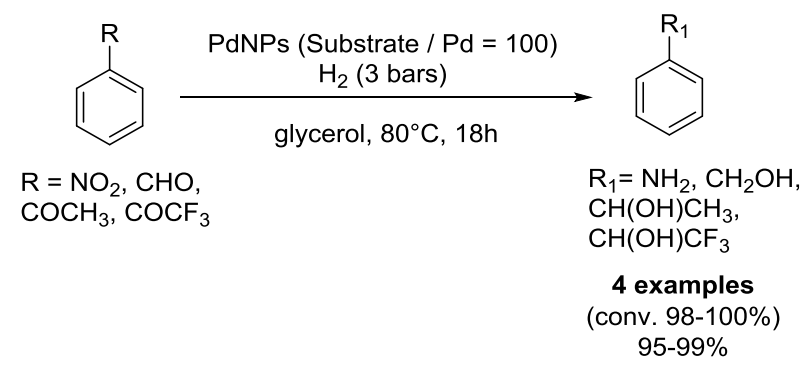

Bimetallic nanoparticles also have been synthetized in alternative solvents for catalytic proposes as hydrogenation reactions. ${ }^{88,190}$ They represent an attractive catalytic system thanks to the synergistic effects between both metal partners; the addition of a "guest" to a "host" metal in a same entity could modify its electronic properties (by charge transfer, orbital hybridization...) 
and/or structure (alloy, core shell...). ${ }^{191}$ In our group, PdCu BMNPs were prepared in glycerol under $\mathrm{H}_{2}$ pressure (as for the Pd NPs previously described) via two main strategies: co- and sequential reduction. ${ }^{126} \mathrm{Pd}(\mathrm{OAc})_{2}$ and $\mathrm{Cu}-\mathrm{TMEDA},\left[\left[\mathrm{Cu}\left(\kappa^{2}-N, N, N^{\prime}, N^{\prime}-\mathrm{TMEDA}\right)(\mu-\mathrm{OH})\right]_{2} \mathrm{Cl}_{2}\right.$, TMEDA $=$ tetramethylethylenediamine $]$ were used as metal precursor and PVP $(\mathrm{Mw}=10000$ $\mathrm{g} / \mathrm{mol}$ ) was used as stabilizer. Under these conditions, only co-reduction processes permitted to obtain BMNPs (see section 2.2.2). The as-prepared BMNPs were tested in the catalytic semihydrogenation of alkynes (Table 1).

Table 1. Semihydrogenation of diphenylacetylene and phenylacetylene catalyzed by $\mathrm{PdCu}$ BMNPs.

\begin{tabular}{|c|c|c|c|c|c|}
\hline \multicolumn{3}{|c|}{$\begin{array}{c}\mathrm{H}_{2}(1 \mathrm{bar}) \\
\text { PdCu BMNPs } \\
\text { (Substrate / Pd }=200) \\
\text { glycerol } \\
80^{\circ} \mathrm{C}, 2 \mathrm{~h}\end{array}$} & \multicolumn{3}{|c|}{$\begin{array}{c}\mathrm{H}_{2}(1 \mathrm{bar}) \\
\mathrm{PdCu} \mathrm{BMNPs} \\
\text { (Substrate } / \mathrm{Pd}=200) \\
\text { glycerol } \\
80^{\circ} \mathrm{C}, 2 \mathrm{~h}\end{array}$} \\
\hline Entry & $\begin{array}{l}\text { Catalyst } \\
\\
(\mathrm{Pd}: \mathrm{Cu} \text { ratio for } \\
\text { BMNPs) }\end{array}$ & $\begin{array}{l}\text { Conv. }(\%)^{\mathrm{b}} \\
\text { (alkene:alkane) }^{\mathrm{b}}\end{array}$ & Entry & $\begin{array}{l}\text { Catalyst } \\
\text { (Pd:Cu ratio for BMNPs) }\end{array}$ & $\begin{array}{l}\text { Conv. }(\%)^{\mathrm{b}} \\
\text { (alkene:alkane) }^{\mathrm{b}}\end{array}$ \\
\hline 1 & PdNPs & $\begin{array}{l}100(0: 100) \\
98(80: 20)^{c}\end{array}$ & 6 & PdNPs & $100(0: 100)$ \\
\hline 2 & CuNPs & 0 & 7 & CuNPs & 0 \\
\hline 3 & PdNPs + CuNPs & $100(0: 100)$ & 8 & PdNPs + CuNPs & $100(17: 83)$ \\
\hline 4 & PdCu BMNPs (1:2) & $37(100: 0)$ & 9 & PdCu BMNPs (1:2) & $56(100: 0)$ \\
\hline 5 & PdCu BMNPs (1:1) & $59(100: 0)$ & 10 & PdCu BMNPs $(1: 1)$ & 84 (100:0) \\
\hline
\end{tabular}


${ }^{a}$ Reaction conditions: $1 \mathrm{mmol}$ of diphenylacetylene or phenylacetylene in the catalytic solution, $0.5 \mathrm{~mol} \%$ total palladium (or copper), 1 bar of $\mathrm{H}_{2}$ at $80{ }^{\circ} \mathrm{C}$ for $2 \mathrm{~h}$. ${ }^{\mathrm{b}}$ Determined by GC-MS using decane as internal standard. ${ }^{c}$ Data using a substrate:palladium ratio of 1000.

With the aim of studying reactivity-structure relationships, the as-prepared BMNPs were tested in the semihydrogenation of phenylacetylene and diphenylacetylene. A similar catalytic behavior was observed for both substrates (Table 1). Actually, $\mathrm{PdCu}$ nanoalloys (obtained for $\mathrm{Pd}: \mathrm{Cu}$ molar ratio $=1: 2$ ) and cluster-in-cluster structure (obtained for $\mathrm{Pd}: \mathrm{Cu}$ molar ratio $=1: 1$ ) gave exclusively the formation of the corresponding alkene (entries 4-5 and 9-10, Table 1), in contrast to the behavior observed for an equimolar mixture of individually prepared monometallic nanoparticles, i.e. PdNPs and CuNPs, mainly obtaining the full hydrogenated alkanes (entries 3 and 8, Table 1). Using low load of palladium (substrate:palladium ratio = 1000), monometallic PdNPs catalyzed the semihydrogenantion of diphenylacetylene favoring in this case the formation of $Z$-stilbene (entry 1). These results proved that even though very small amount of palladium is present on the surface of MNPs, the semihydrogenation reaction of alkynes is possible, in agreement with the behavior observed using PdCu BMNPs.

4. Pd-based nanoparticle leveraged coupling reactions.

Catalysis offers a plethora of new tools not only to activate chemical bonds, but also to introduce new chemical functions in a tailored fashion. The selective formation of new $\mathrm{C}-\mathrm{C}, \mathrm{C}-\mathrm{N}, \mathrm{C}-\mathrm{O}$, $\mathrm{C}-\mathrm{S}$ or $\mathrm{C}-\mathrm{P}$ bonds through catalyzed processes represents unrivalled means in synthesis with applications in many other disciplines, such as material science, biomedicine, and electronics. ${ }^{1,192-194}$ Transition metals are among the most versatile elements for enabling these coupling reactions by providing energetically affordable reaction pathways. ${ }^{195,196}$ Nanocatalysis 
permits to envisage novel avenues towards substrate activation by a combination of both homogeneous and heterogeneous reaction manifolds. ${ }^{197-200}$ Among transition metals, Pd exhibits a prevalent role notably due to its catalytic activity towards $\mathrm{C}-\mathrm{C}$ cross-coupling reactions and related transformations. ${ }^{3,198,201-204}$ Pioneering studies by Beletskaya, ${ }^{205}$ Reetz $^{206}$ and de Vries ${ }^{207}$ showed that Pd nanoparticles can activate aryl halide substrates (iodides and, in some cases, bromides) at $\mathrm{ppm}$ concentrations, enabling $\mathrm{C}\left(\mathrm{sp}^{2}\right)-\mathrm{C}\left(\mathrm{sp}^{2}\right)$ cross-coupling reactions (Heck and Suzuki-Miyaura) at catalyst turnover numbers as high as 540,000. Furthermore, the increasing of the catalyst turnover with the decreasing $\mathrm{Pd} /$ substrate ratios suggested that a homogeneous mechanism by metal leaching could be operative for the Heck reaction. ${ }^{208-210}$ These very low catalyst loadings have enabled a direct entry towards nanoparticle-leveraged sustainable approaches ${ }^{199,211,212}$ via immobilization on solid supports ${ }^{213}$ or biomass-derived solvents as liquid phases. ${ }^{214}$ Astruc and co-workers have recently showed that PdNPs supported on supramolecular dendrimeric assemblies featuring tri(ethylene glycol) tethers exhibit high catalytic activities and turnover numbers at low catalytic loadings $(0.01 \mathrm{~mol} \%)$ for Suzuki-Miyaura reactions. ${ }^{210,215}$ From a mechanistic point of view, many efforts have been addressed with the aim of elucidating the nature of the active palladium species involved in C-C couplings: homogeneous (reagents interact with one active site) or heterogeneous (reagents interact with neighboring sites), based on the modern definition proposed by Schwartz ${ }^{216}$ surpassing the classical definition based on the catalyst solubility. Studies for distinguishing the catalyst nature include kinetics, poison experiments $\left(\mathrm{Hg}, \mathrm{CS}_{2} \ldots\right)$, polymer test, hot filtration, catalyst characterization after catalysis. $38,217,218$

Several reports on the utilization of polyol medium both as reducing agent of metal precursor, as well as matrixes for nanoparticle immobilization have been described in the literature. ${ }^{219}$ The low 
cost, nontoxicity and eco-friendliness of most polyols (glycerol, PEG) makes them solvents of choice for a number of catalyzed reactions, with significant advantages over ionic liquids and perfluorinated solvents. ${ }^{220}$ Moreover, the polarity of polyol media has non-negligible effects in facilitating catalytic activity. ${ }^{172}$ In particular, polysaccharides are adequate polyol materials exhibiting high stability and recyclability for catalysis. ${ }^{221,222}$ This section intends to give an overview and critical summary of research achievements in the use of both polyol medium and polyols embedded into polymeric matrixes in coupling reactions.

\subsection{Dimerization of alkynes.}

Pd-catalyzed dimerization of terminal alkynes provides a straightforward method for the synthesis of 1,4-disubstituted enynes. ${ }^{223}$ However, very few reports in polyol leveraged nanocatalytic systems have been reported thus far. For instance, Firouzabadi, Iranpoor and coworkers have reported the dimerization reaction of phenylacetylene in air under ligand- and amine-free conditions using PEG400 as solvent at $100{ }^{\circ} \mathrm{C}$ to furnish the homo-coupled $E$-enyne product in $70 \%$ isolated yield (Scheme 17). The reaction proceeds in air with high regio-and stereoselectivity. ${ }^{224}$ Further mechanistic studies for this transformation are required to identify the active catalytic species and the catalytic regime.

Scheme 17. Pd-catalyzed dimerization of phenylacetylene. ${ }^{224}$

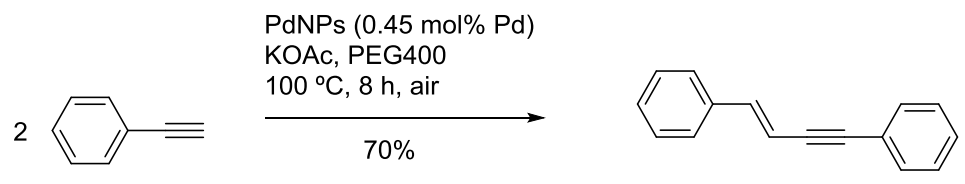




\section{2. $\mathrm{C}(\mathrm{sp})-\mathrm{C}\left(\mathrm{sp}^{2}\right)$ Sonogashira cross-coupling}

The Sonogashira coupling is a Pd-catalyzed cross-coupling reaction that forms a new $\mathrm{C}-\mathrm{C}$ bond between a terminal alkyne and an $\mathrm{sp}^{2}$-carbon halide (most notably, aryl or vinyl). ${ }^{225}$ This reaction was initially reported with a $\mathrm{Cu}(\mathrm{I})$ co-catalyst in the presence of a base under anaerobic and anhydrous conditions, but other methods have been later reported to overcome these limitations. For the $\mathrm{Cu}$-free version in particular, the activation of the terminal alkyne derivative is achieved by formation of $\mathrm{Pd}(\mathrm{II})$ monoacetylide and $\mathrm{Pd}(\mathrm{II})$ bisacetylide complexes in the presence of base, which are competent species to undergo the corresponding transmetallation under mild reaction conditions. The proposed catalytic cycle encompasses an oxidative addition of the aryl halide to the zero-valent Pd catalyst, generating a $\mathrm{Pd}(\mathrm{II})$ complex which is involved in the transmetallation step with the $\operatorname{Pd}(\mathrm{II})$ bisacetylide complex, followed by reductive elimination to deliver the disubstituted alkyne product and the regeneration of the zero-valent Pd species completing the catalytic cycle (Figure 20). ${ }^{226}$

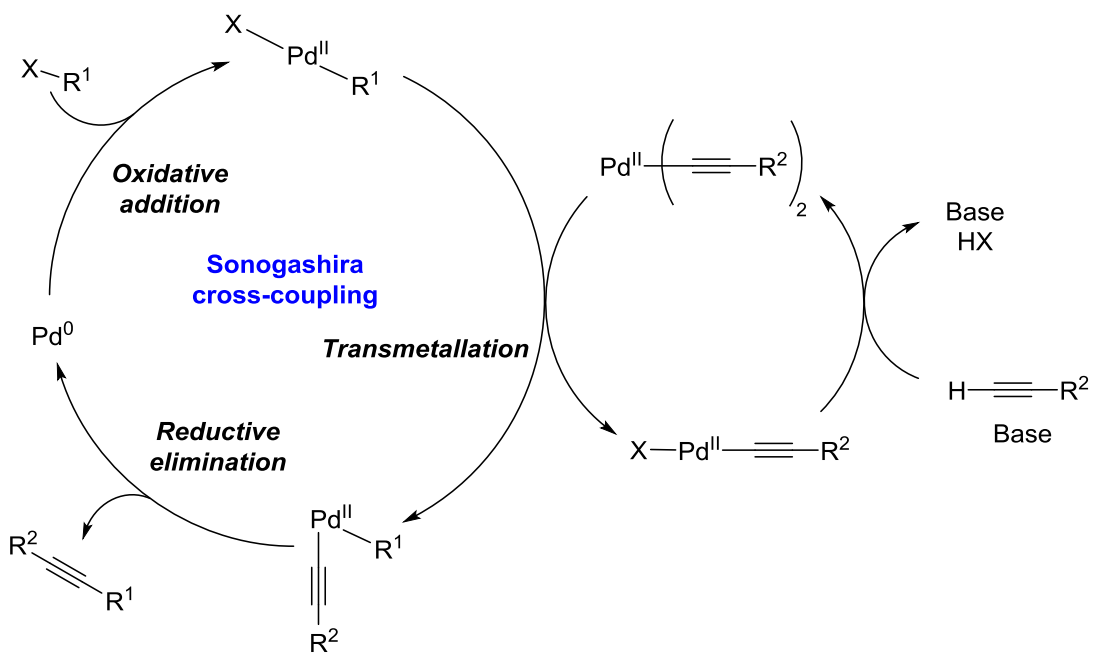

Figure 20. Proposed mechanism for the $\mathrm{Cu}$-free Sonogashira cross-coupling reaction based on molecular palladium species. 
Homogeneous catalysts were first described as active catalytic species for this transformation. In 2006, Corma, García and co-workers described the use of a covalently immobilized oxime carbapalladacycle on PEG (averaged Mw $6000 \mathrm{Da}$ ) as a reusable catalytic system for a copperfree Sonogashira couplings (up to 10 recyclings). ${ }^{219}$ Despite the stability of the anchored molecular catalyst on heating over extended periods in PEG, it decomposed during catalysis to form palladium nanoparticles which were active towards copper-free Sonogashira couplings and enabled catalyst recycling up to 10 times with no significant decrease in activity. ${ }^{219}$ The authors highlight that the reactants employed during catalysis can have an impact in the size of the in situ formed nanoparticles, which can lead to broad particle size distribution. However, the most active in situ formed nanocatalytic systems were those featuring average particle size of $6 \pm 2$ $\mathrm{nm}^{219}$

Another highly activity heterogenized $\mathrm{Cu}$-free catalytic system with agarose as support for the Sonogashira reaction with low catalyst loading $(0.5 \mathrm{~mol} \%)$ has been recently described by Firouzabadi, Iranpoor and co-workers. ${ }^{227}$ Despite the fact that the authors describe a stabilizerfree catalytic system for this cross-coupling reaction of various aryl halides with phenyl acetylene in PEG400, the effect of the polyol polymeric support, namely agarose as stabilizer for the palladium nanoparticles is not negligible (Scheme 18). ${ }^{227}$

Scheme 18. Sonogashira reaction of aryl halides with phenyl acetylene catalyzed by agarose supported PdNPs in PEG 400 (agarose is a linear polymer constituted of repeating units of 4- O- $\beta$ - D- galactopyranosyl- 3,6- anhydro- L- galactose). ${ }^{227}$ 


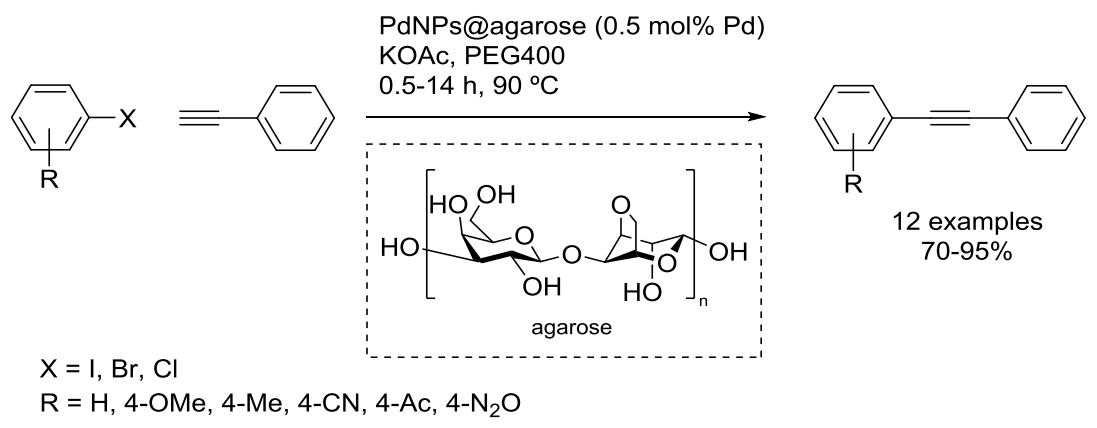

The same authors have also reported a similar strategy for the use of edible gelatin as both reducing agent of Pd(II) salt precursors and support for PdNPs exhibiting high activity towards amine-free catalyzed Sonogashira reaction. Thus, (hetero)aryl halides or even $\beta$-bromo styrene reacted with phenylacetylene at $100{ }^{\circ} \mathrm{C}$ in PEG400 medium under Ar to yield the desired crosscoupling products in high selectivity and moderate to high yields (Scheme 19). ${ }^{224}$

Scheme 19. Amine-free Sonogashira reaction in PEG medium. ${ }^{224}$

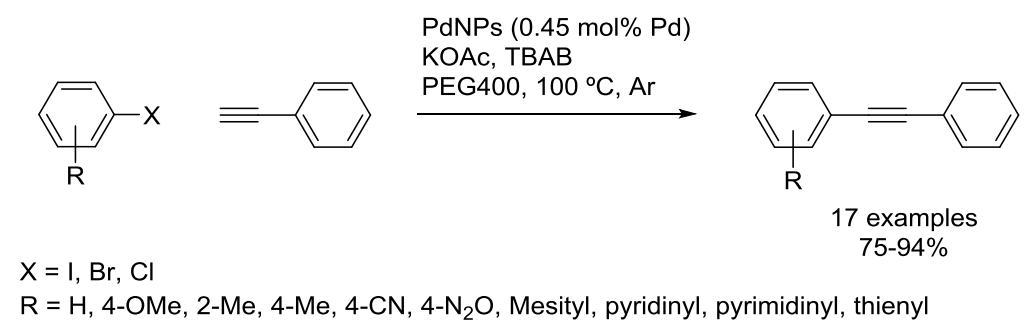

Moreover, EG has also been used as reducing and dispersing agent for the preparation of highly efficient Sonogashira nanocatalysts based on PdCo BMNPs grafted on three-dimensional graphene nanocomposites by Dabiri and co-workers. ${ }^{228}$

4.3. $\mathrm{C}\left(\mathrm{sp}^{2}\right)-\mathrm{C}\left(\mathrm{sp}^{2}\right)$ Hiyama-Denmark cross-coupling 
Progress on the classical fluoride-assisted Pd-catalyzed $\mathrm{C}\left(\mathrm{sp} / \mathrm{sp}^{2} / \mathrm{sp}^{3}\right)-\mathrm{C}\left(\mathrm{sp}^{2}\right)$ Hiyama crosscoupling $^{229}$ has yielded safe alternatives that preclude the risks associated to the handling of fluoride. ${ }^{230,231}$ The key step of this reaction involves the formation of a pentavalent silicon species that is capable to undergo transmetalation with the Pd center. In the classical version, the formation of such species was driven via the formation of pentacoordinated fluorosilanes due the high affinity of $\mathrm{F}$ and $\mathrm{Si}$, but organosilanols either in the presence of Brønsted bases or as their silanolate salts are also competent species to undergo transmetalation (Figure 21). ${ }^{232}$

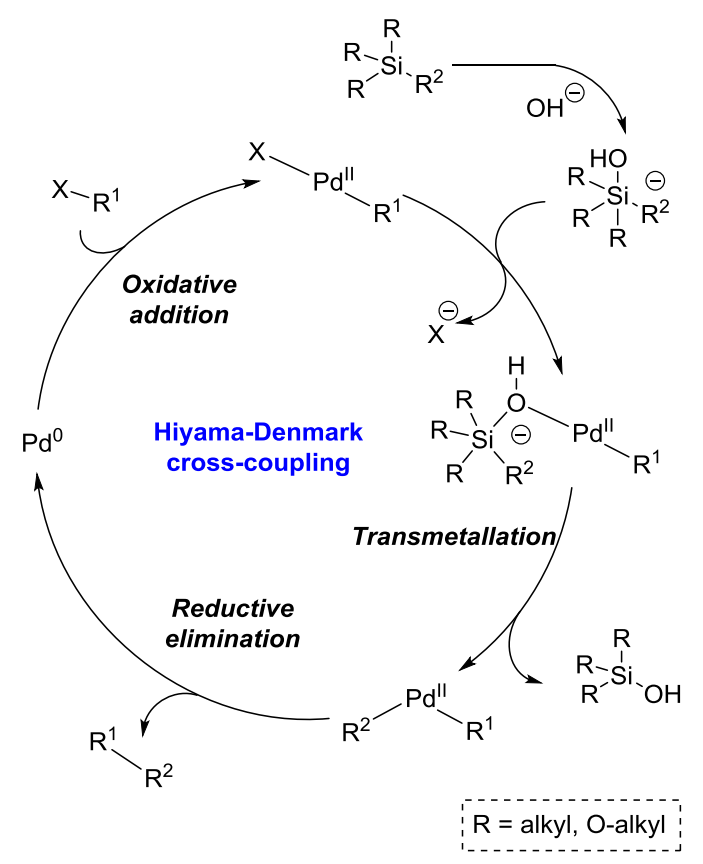

Figure 21. Pd-catalyzed Hiyama-Denmark reaction mechanism based on molecular palladium species.

The postulated Hiyama-Denmark cross-coupling reaction mechanism was believed to operate under homogenous regime; however, Nájera and co-workers identified the decomposition of $\mathrm{Pd}(\mathrm{OAc})_{2}$ or even 4-hydroxyacetophenone oxime-derived palladacycles towards the formation of 
PdNPs at catalyst loadings up to $0.01 \mathrm{~mol} \%$ when tetrabutylammonium bromide was used as stabilizer. ${ }^{233}$ Nanoparticles can act as homogeneous catalysts (reservoirs of active molecular species), surface reactivity, or a combination both processes.

For instance, Sarkar and co-workers have described the synthesis of PdNPs using a tungsten carbene complex as reducing agent and PEG6000 as capping agent in water. ${ }^{234}$ The as-prepared nanocatalysts (average particle size $9.70 \mathrm{~nm}$ ) catalyzed the Hiyama-Denmark cross-coupling reaction with low catalyst loadings $(1 \mathrm{~mol} \%)$ in air (Scheme 20$).^{234}$

Scheme 20. PdNPs leveraged Hiyama cross-coupling of arylsiloxanes with aryl bromides. ${ }^{234}$

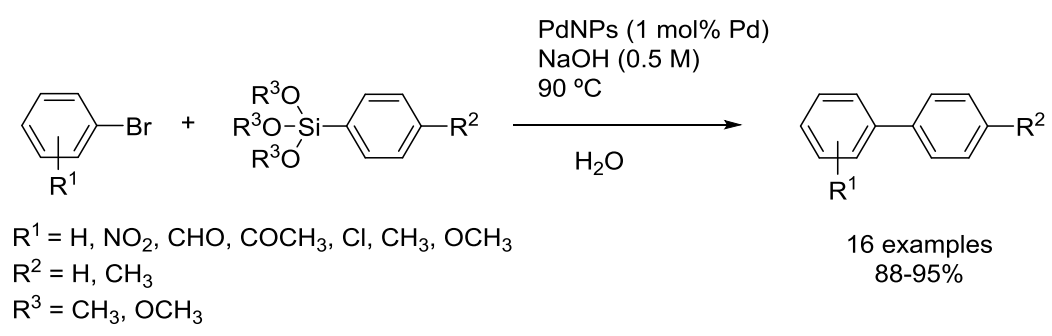

Recent studies on the Hiyama-Denmark cross-coupling leveraged by preformed PdNPs in glycerol medium shed light on the key role of different additives; not only to ensure the kinetic stabilization of the colloidal catalyst systems, but also the coordination mode between these additives and the metal surface modifying the reaction manifold. ${ }^{235}$ Thus, our group has described a highly active preformed Pd nanocatalyst system featuring small PdNPs (mean diameter $1.8 \pm 0.4 \mathrm{~nm}$ ) using TPPTS in glycerol. ${ }^{235}$ TPPTS favors the leaching and further stabilization of Pd(II) molecular complexes thanks to the strong P-Pd bond. ${ }^{235}$ The as-prepared catalytic system exhibited substrate-dependent chemoselectivity towards the expected HiyamaDenmark cross-coupling enabling the synthesis of unsymmetrical bis-aryls from 
trimethoxyphenylsilane and bromoarenes containing both electron donating and withdrawing groups $\left(p-\mathrm{OMe}, p-\mathrm{CN}, p-\mathrm{CF}_{3}\right){ }^{235}$

PdNPs leveraged versions of the classical fluoride-activated Hiyama coupling have also been described by Sarkar and co-workers with nanocatalysts generated from $\mathrm{K}_{2}\left[\mathrm{PdCl}_{4}\right]$ in PEG600 medium at $70{ }^{\circ} \mathrm{C}$ for 15 min. ${ }^{236}$ The colloidal nanocatalyst suspensions were active at room temperature for the transformation of aryltrialkoxysilanes and benzyl halides into diarylmethanes in good to excellent yields (Scheme 21). ${ }^{236}$

Scheme 21. PdNPs catalyzed Hiyama cross-coupling reaction of aryltrialkoxysilanes and benzyl halides. $^{236}$
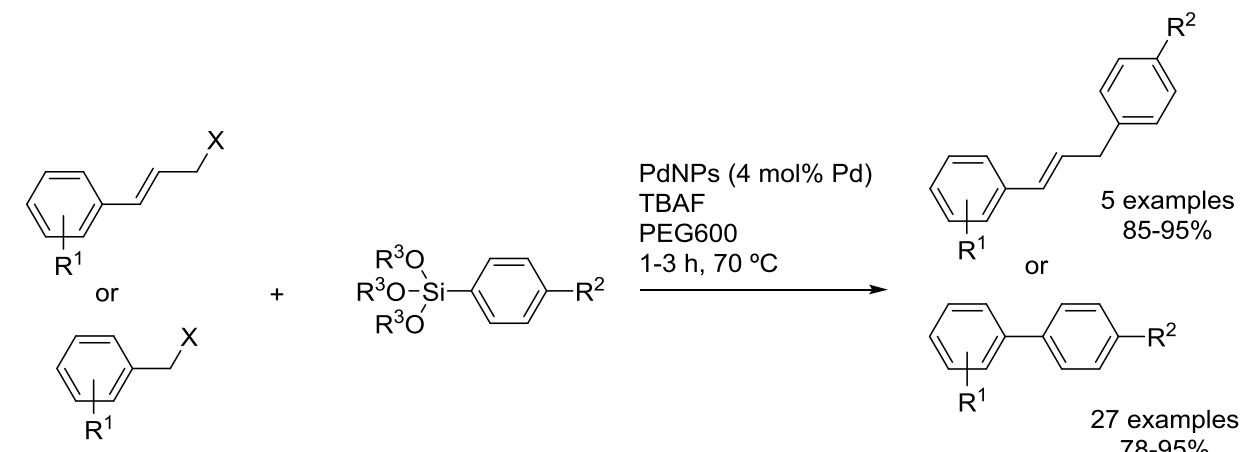

$$
\begin{aligned}
& \mathrm{R}^{1}=\mathrm{H}, 3-\mathrm{Cl}, 3-\mathrm{OMe}, 4-\mathrm{OMe}, 4-\mathrm{CF}_{3}, 4-\mathrm{F}, 4-\mathrm{CHO}, 1 \text {-naphth, 2-naphth, } \mathrm{COCH}_{3} \\
& \mathrm{R}^{2}=\mathrm{H}, \mathrm{OMe} \mathrm{Me} \\
& \mathrm{R}^{3}=\mathrm{CH}_{2} \mathrm{CH}_{3}, \mathrm{CH}_{3} \\
& \mathrm{X}=\mathrm{Cl}, \mathrm{Br} \text {, OTs }
\end{aligned}
$$

\section{4. $\mathrm{C}\left(\mathrm{sp}^{2}\right)-\mathrm{C}\left(\mathrm{sp}^{2}\right)$ Heck-Mizoroki cross-coupling}

The Heck-Mizoroki cross-coupling reaction was the first example of a Pd catalyzed C( $\left(\mathrm{sp}^{2}\right)$ $\mathrm{C}\left(\mathrm{sp}^{2}\right)$ bond-forming reaction between aryl or vinyl halides and olefins. ${ }^{237,238}$ The initially proposed $\mathrm{Pd}(0) / \mathrm{Pd}(\mathrm{II})$ catalytic cycle under homogeneous conditions encompasses the oxidative 
addition an aryl or vinyl halide to a zero-valent Pd center to generate an electrophilic $\mathrm{Pd}(\mathrm{II})$ species that can coordinate with an olefin via a $\pi$ interaction, followed by alkene insertion in the $\mathrm{Pd}-\mathrm{C}\left(\mathrm{sp}^{2}\right)$ bond via a syn addition step. The generated intermediate undergoes then a $\beta$-hydride elimination to deliver the $\mathrm{C}\left(\mathrm{sp}^{2}\right)-\mathrm{C}\left(\mathrm{sp}^{2}\right)$ cross-coupling product and the residual hydrido(halo)palladium(II) complex regenerates the initial $\mathrm{Pd}(0)$ by reductive elimination. In the course of the reaction, the base is stoichiometrically consumed (Figure 22).

However, the formation of nanoparticles in Heck-Mizoroki cross coupling reactions has been evidenced in many literature reports most of them under ligand-free conditions. ${ }^{209}$ Palladium is known to give unstable and polydisperse solutions in the absence of polymers or amphiphilic stabilizers encompassing catalyst deactivation and precipitation of Pd black aggregates. ${ }^{209,239}$

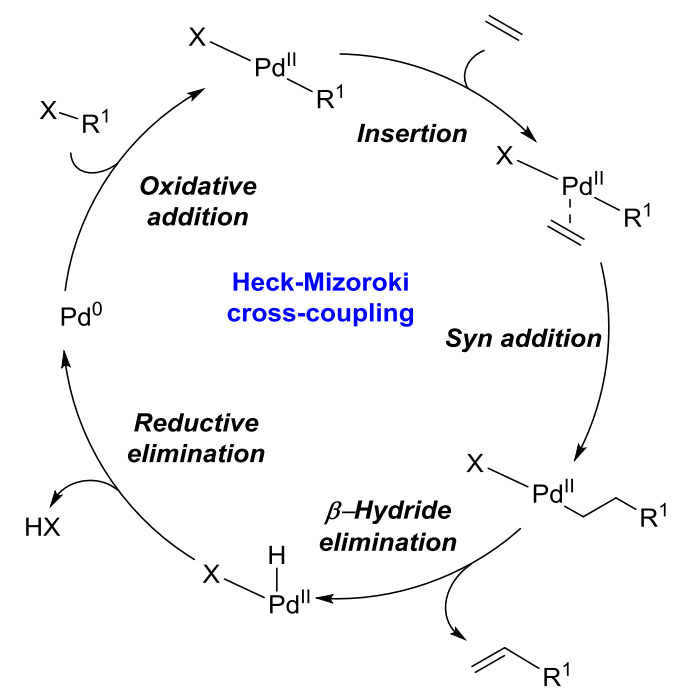

Figure 22. Classical Pd-catalyzed Heck-Mizoroki reaction mechanism based on molecular palladium species. 
The obtention of monodispersed systems is thus highly envisaged for enhancing the catalyst activity. In this context, the use of polyol medium in combination with palladium salts or organometallic complexes leads to the formation of zero-valent Pd nanoparticles both under classical thermal treatment and ball-milling conditions. ${ }^{59,221,240}$ Lamaty at al. have reported a phosphine-free Mizoroki-Heck reaction using ball-milling in PEGs and methyl ethers derivatives of different molecular weights, obtaining good to excellent yields of cross-coupling products. ${ }^{59}$ Notably, this activation technique allowed the formation of PdNPs of $4 \pm 2 \mathrm{~nm}$ (average diameter size) from $\operatorname{Pd}(\mathrm{OAc})_{2}$ in the time course of the reaction when sodium formate was used (as characterized by TEM analysis of the reaction crudes). ${ }^{59}$

Another example of Heck-Mizoroki cross-coupling reaction catalyzed by PdNPs supported on optically-active polymeric supports has been described by Frey and co-workers ${ }^{240}$ The authors described the encapsulation of PdNPs of $1-5 \mathrm{~nm}$ average sizes from $\mathrm{PdCl}_{2}$ using $\mathrm{NaBH}_{4}$ as reducing agent and the amphiphilic hyperbranched polyglycerol support as template. 2,3Dihydrofuran and aryl triflate were efficiently coupled to furnish racemic 2-aryl-2,3dihydrofuran in the presence of $N, N$-diisopropylethylamine at $30^{\circ} \mathrm{C}$ for extended periods of time $(4$ days $) .{ }^{240}$

More recently, Evangelisti and co-workers have described the immobilization of small PdNPs obtained by metal vapor synthesis technique into poly(4-vinylpyridine)-based porous monolith for continuous-flow Mizoroki-Heck reaction applications. ${ }^{241}$ The synthesis of the catalytic devices was carried out by radical co-polymerization of the metal-embedded monomer with ethylene glycol dimethylacrylate in the presence of PEG as porogenic agent inside stainless-steel columns. The as-prepared reactors containing highly dispersed PdNPs (average particle size $=$ $2.3 \mathrm{~nm}$ ) showed long life-time ( $>65 \mathrm{~h})$ and very low Pd leaching $(<2 \mathrm{ppm})$ towards continuous- 
(Scheme 22). ${ }^{241}$

Scheme 22. Synthesis of Pd supported nanocatalysts on a porous PVPy monolith. Reprinted with permission from Elsevier, ${ }^{241}$ license number 4558310654891.

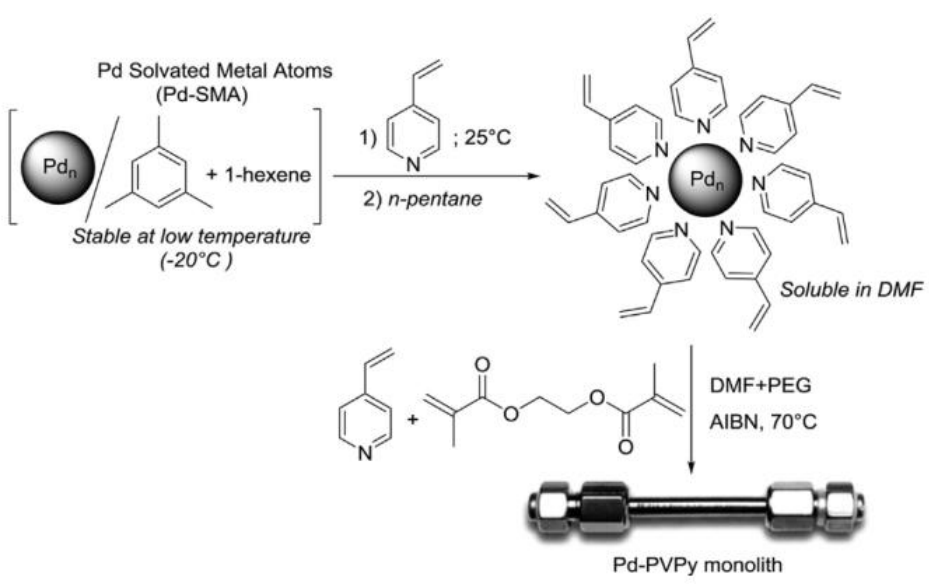

An alternative heterogenization protocol for the preparation of PdNPs immobilized on amphiphilic polyethylene glycol- block- polycitric acid (PCA- $b$ - PEG)- functionalized magnetite composites has been reported by Rezaei and co-workers. ${ }^{242}$ The as-prepared hybrid magnetite spheres (15 $\mathrm{nm}$ average size) were able to trap $\mathrm{Pd}(\mathrm{II})$ species via dendrimer complexation with embedded free carboxylic acid residues which led to dendritic shell immobilized PdNPs as observed by TEM (Scheme 23). ${ }^{242}$ The catalyzed Mizoroki-Heck crosscoupling reaction with the as-prepared catalytic composite represents an example of a sustainable 
method with enhanced catalyst recovery by applying an external magnetic field and reusability for more than ten consecutive runs exhibiting only minor losses in activity. ${ }^{242}$

Scheme 23. Synthetic route employed for immobilization of PdNPs on $\mathrm{Fe}_{3} \mathrm{O}_{4} @$ PCA- $b$ - PEG magnetic supports. Reproduced with permission from John Wiley and Sons, ${ }^{242}$ license number 
4558311368053.

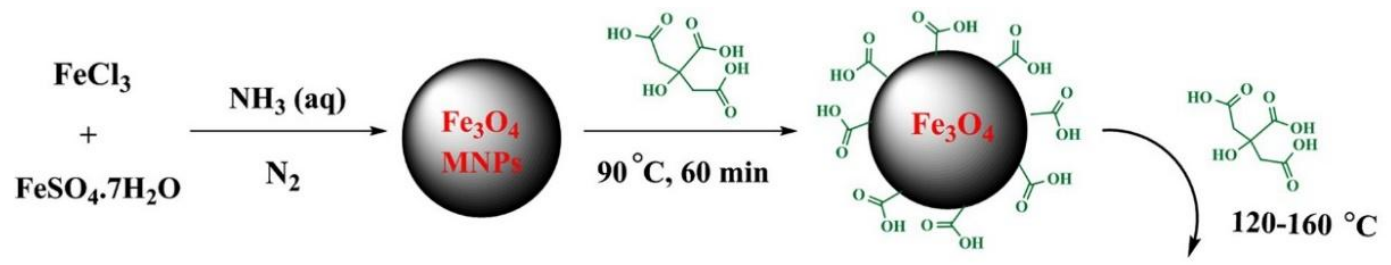

$\mathrm{Fe}_{3} \mathrm{O}_{4}-(\mathrm{COOH})_{\mathrm{n}}$

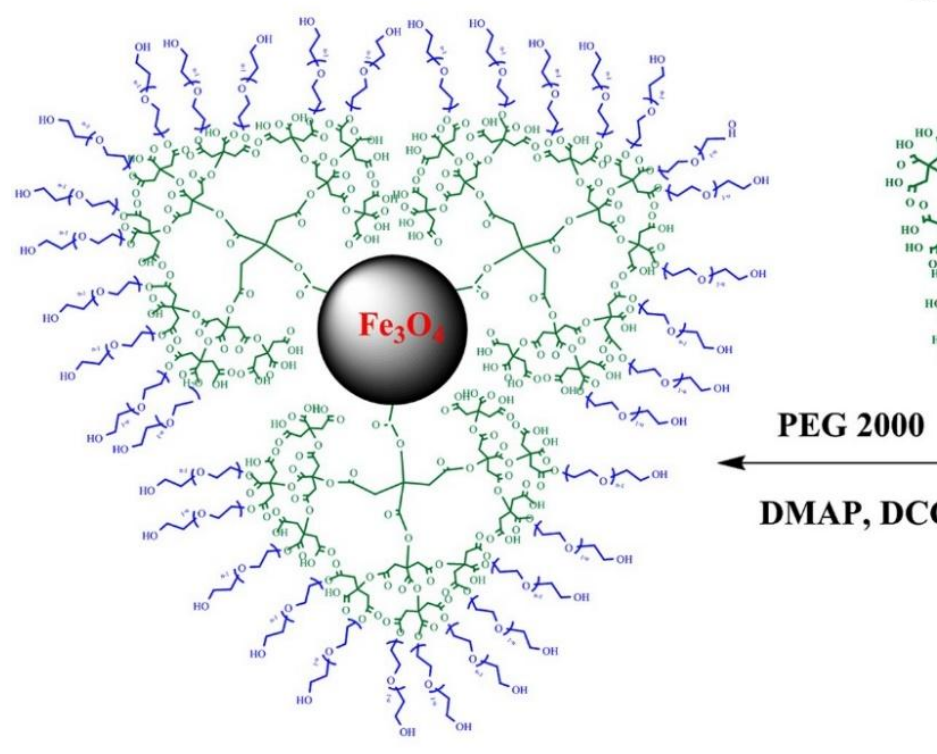

$\mathrm{Fe}_{3} \mathrm{O}_{4} @$ PCA- $b$-PEG

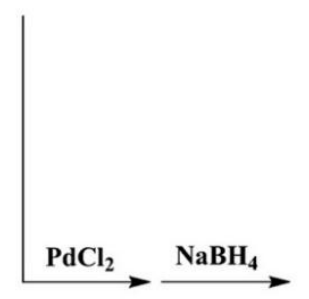

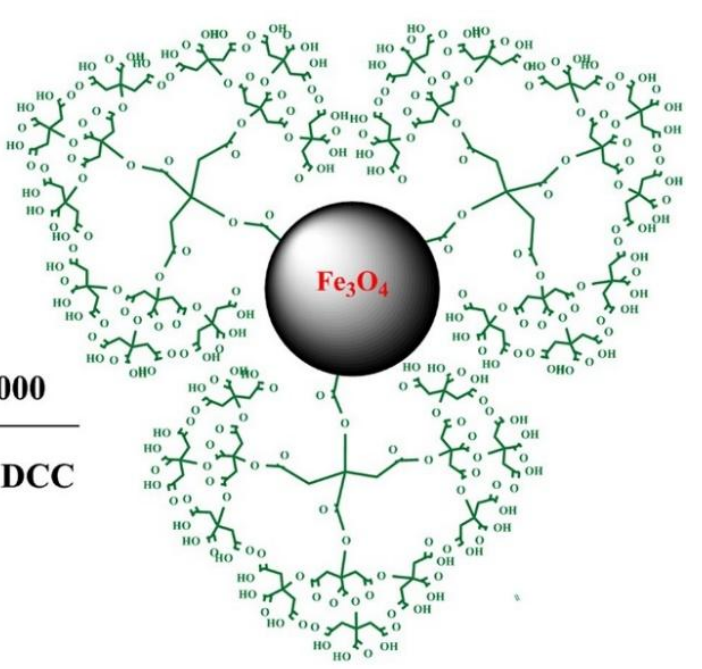

$\mathrm{Fe}_{3} \mathrm{O}_{4} @ P C A$

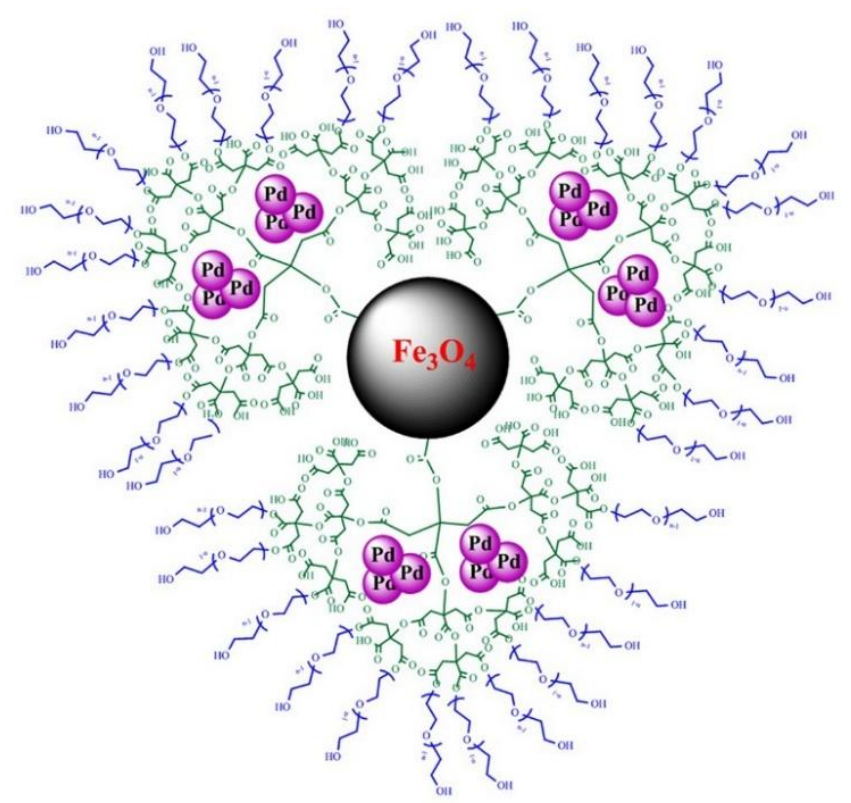

$\mathrm{Fe}_{3} \mathrm{O}_{4} @$ PCA/Pd(0)-b-PEG

4.5. $\mathrm{C}\left(\mathrm{sp}^{2}\right)-\mathrm{C}\left(\mathrm{sp}^{2} / \mathrm{sp}^{3}\right)$ Suzuki-Miyaura cross-coupling. 


\subsubsection{In PEG and glycerol media.}

First described in 1979, the Suzuki-Miyaura cross-coupling reaction is a Pd-catalyzed transformation of a boronic acid and an aryl halide that generates a product featuring a new $\mathrm{C}\left(\mathrm{sp}^{2}\right)-\mathrm{C}\left(\mathrm{sp}^{2}\right)$ or $\mathrm{C}\left(\mathrm{sp}^{2}\right)-\mathrm{C}\left(\mathrm{sp}^{3}\right) \cdot{ }^{243}$ Among all Pd-catalyzed transformations, the Suzuki-Miyaura cross-coupling reaction has flourished in the last decades enabling new efficient methodologies for the creation of $\mathrm{C}\left(\mathrm{sp}^{2}\right)-\mathrm{C}\left(\mathrm{sp}^{2}\right)$ bonds. This is in part thanks to the large availability of boronic acid derivatives now available from commercial sources.

A key elementary step of this transformation has been classically described as an oxidative addition of an aryl halide to a zero-valent Pd center taking place under a homogenous regime. However, Razler and co-workers identified the formation of in situ-generated $15 \pm 5 \mathrm{~nm}$ PdNPs from $\mathrm{Pd}(\mathrm{OAc})_{2}$ after only 1 min of reaction in PEG media by TEM analysis. ${ }^{244}$

Thus, the impact of the in situ formed nanoparticles on the operating catalytic cycles needed further studies. Corma, García and co-workers reported that small size nanoparticles have a key role in facilitating the oxidative addition involving the formation of aryl Pd(II) intermediates, ${ }^{219}$ but the nanocatalysts regimes, i.e. reservoirs of molecular species or surface heterogeneous-like catalysts remain still not clear. Catalyst heterogenization on inorganic supports $\left(\mathrm{SiO}_{2}, \mathrm{Al}_{2} \mathrm{O}_{3}\right.$, zeolites) or organic polymers has received an increasing interest in the scientific community, with special emphasis on entrapped nanoparticles in polymeric matrixes. ${ }^{197,245}$

Furthermore, Pd nanocatalysis enables one-pot, two-step processes to transform aryl and benzyl halides into unsymmetrical biaryls and diarylmethanes via sequential borylation-Suzuki-Miyaura coupling as reported by Sarkar and co-workers. ${ }^{246}$ The authors described the synthesis of nanoparticles of $6 \mathrm{~nm}$ average diameter size by thermal treatment at $70{ }^{\circ} \mathrm{C}$ of $\mathrm{K}_{2}\left[\mathrm{PdCl}_{4}\right]$ in PEG 
for $15 \mathrm{~min}$. The preformed nanoparticles catalyzed the synthesis of aryl/benzyl boronates from their chloro and bromo precursors (in low catalyst loadings $2-3 \mathrm{~mol} \%$ ). ${ }^{246}$ This example highlights the fact that planar trigonal B centers preclude the in situ transmetallation of the boronate ester products formed in moderate to good yields (50-78\%) using KOAc as mild base. However, the addition of $\mathrm{CsF}$ in combination with the aryl halide of choice to the reaction medium enabled the Suzuki-Miyaura cross coupling reaction manifold. In particular, the high affinity of fluoride and boron might trigger the formation of the corresponding ate complex, leading to the pyramidalization of boron. The boron center is then poised to undergo transmetallation with Pd, which is the elementary step needed for the Suzuki-Miyaura crosscoupling reaction manifold. This sequential strategy with a preformed Pd nanocatalyst was applied in one-pot towards the synthesis of both unsymmetrically and symmetrically substituted bis-aryls and diarylmethanes (55-75\% isolated yields). ${ }^{246}$

The efficiency of similar phosphine-free Suzuki-Miyaura cross-coupling of (hetero)aryl bromides with (hetero)arylboronic acids has been carried out at preparative scale in PEG medium allowing the isolation of the desired bis-aryl products in good to excellent yields after an aqueous workup. $^{244}$

Moreover, the advantages of glycerol for the immobilization of the Pd nanocatalysts in liquid phases has been extensively studied in our group. Controlled syntheses in glycerol of preformed nanoparticles were carried out starting from metal salts or organometallic complexes under $\mathrm{H}_{2}$ pressure (1 to 3 bar) with different stabilizers P- and N-containing stabilizers, such as TPPTS ${ }^{187}$ or cinchona-based alkaloids (cinchonidine, quinidine). ${ }^{187,235}$ The as-prepared zero-valent homogeneously dispersed PdNPs exhibited spherical morphology and mean diameters in the range of $1.4-2.1 \mathrm{~nm}$, as determined by TEM analyses. The kinetically stabilized colloidal 
solutions with TPPTS showed excellent catalytic properties towards facilitating cross-coupling reactions and prolonged activity upon recycling preserving its selectivity. ${ }^{74,187,235}$

The literature reports on ligand-free Suzuki-Miyaura cross-coupling reactions under aerobic conditions attire great attention, as the presence of $\mathrm{O}_{2}$ has thus far been deleterious towards the cross-coupling reaction. For instance, Liu and co-workers report ligand-free in situ generated nanocatalysts to work under aerobic conditions in PEG400 at room temperature for the coupling of aryl chlorides and boronic acids. ${ }^{247}$ The in situ generated nanoparticles of $1.5 \mathrm{~nm}$ in average diameter size were more active than preformed ones. This can be explained by Ostwald ripening or agglomeration under the reported ligand-free conditions. ${ }^{247}$ Despite the authors do not comment on the solubility of $\mathrm{O}_{2}$ gas in PEG400 used as solvent for this transformation, this might be a key parameter to preclude competing side reactions such as the homo-coupling of boronic acid derivatives. Indeed, Moreno-Mañas and co-workers ${ }^{248}$ and Amatore, Jutand and collaborators $^{249}$ reported the key role of $\mathrm{O}_{2}$ in the formation of palladium (II) peroxo species capable of undergoing two successive transmetallation events with boronic acid derivatives to generate the corresponding homo-coupling products in a competitive fashion to the analogous transmetallation to a $\mathrm{Pd}(\mathrm{II})$ center generated by the oxidative addition of an aryl halide to $\operatorname{Pd}(0)$ in the classical Suzuki-Miyaura cross-coupling reaction (Figure 23). Progress on the mechanistic insight of this elementary step has revealed that pre-transmetallation complexes containing $\mathrm{Pd}-\mathrm{O}-\mathrm{B}$ linkages are kinetically competent species poised for transmetallation. ${ }^{250,251}$ Despite the 0.5 equiv. excess of boronic acid derivatives used by the authors compared to aryl halides, the role of the ionic liquid polymer in precluding the homo-coupling reaction manifold merits further studies. 


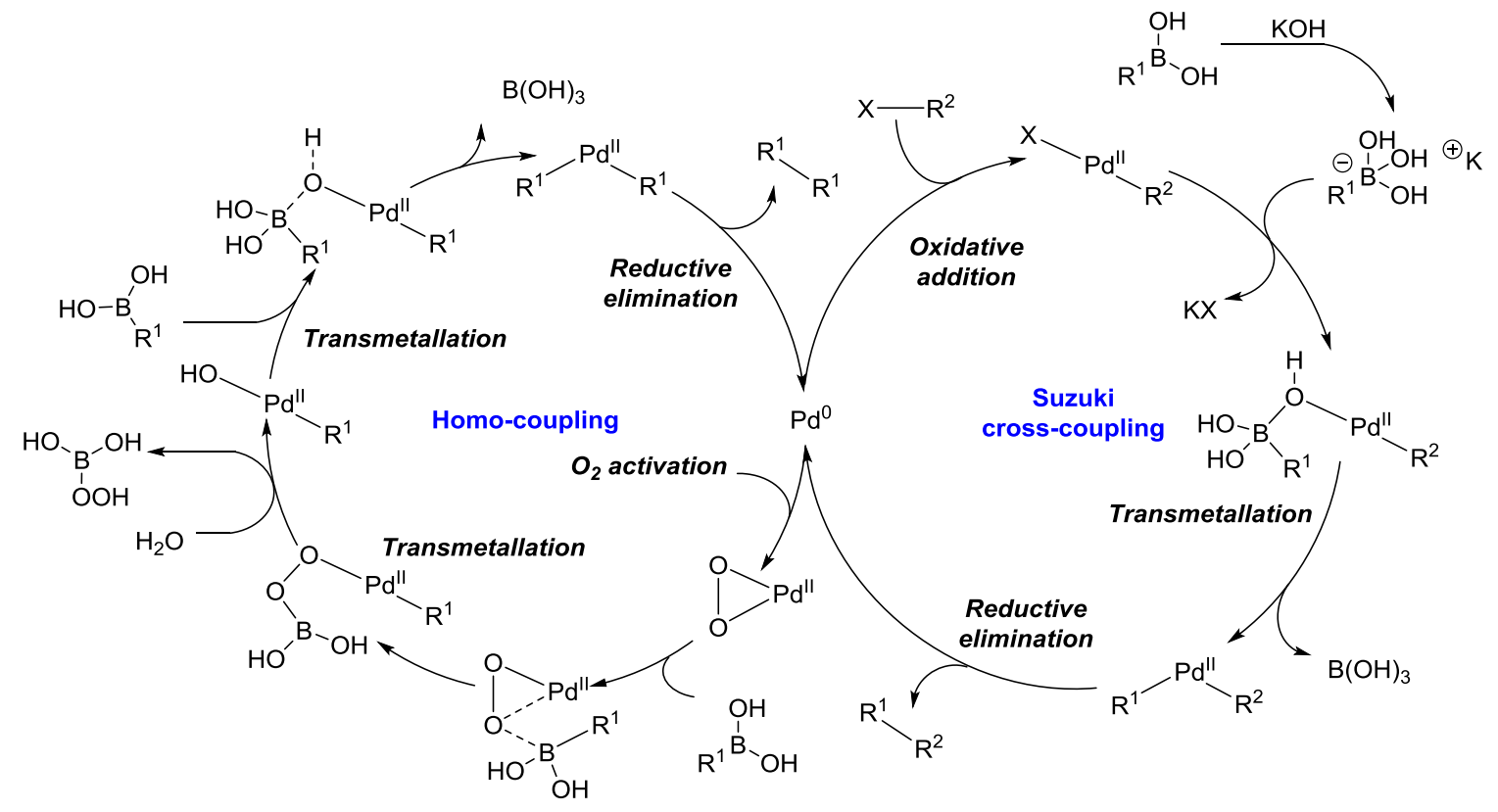

Figure 23. Competing cross-coupling vs homo-coupling manifolds catalyzed by molecular palladium species. The oxidative addition of aryl halides to $\operatorname{Pd}(0)$ is the rate determining step.

Another Suzuki-Miyaura cross-coupling reaction carried out with high selectivity under aerobic conditions has been reported by Zakharova and co-workers with a catalytic system composed of Pd aggregates of $2 \mathrm{~nm}$ in average size, sodium dodecylsulfate as surfactant in a solvent mixture of polyethylene glycol and water. ${ }^{252}$

\subsubsection{Solid-supported nanocatalysts.}

A polystyrene-poly(ethylene glycol) resin has been utilized by Bradley and co-workers for the immobilization of $\operatorname{Pd}(0)$ nanoparticles of $7.4 \pm 1.4 \mathrm{~nm}$ in average size formed by hydrazine hydrate reduction in methanol of the impregnated $\mathrm{Pd}(\mathrm{OAc})_{2}$ precursor in the polymeric matrix 
(Figure 24). ${ }^{253}$ These heterogenized nanocatalysts efficiently catalyzed the Suzuki-Miyaura cross-coupling of electron-donating and electron-withdrawing aryl bromides with boronic acids $(62-99 \%) .{ }^{253}$

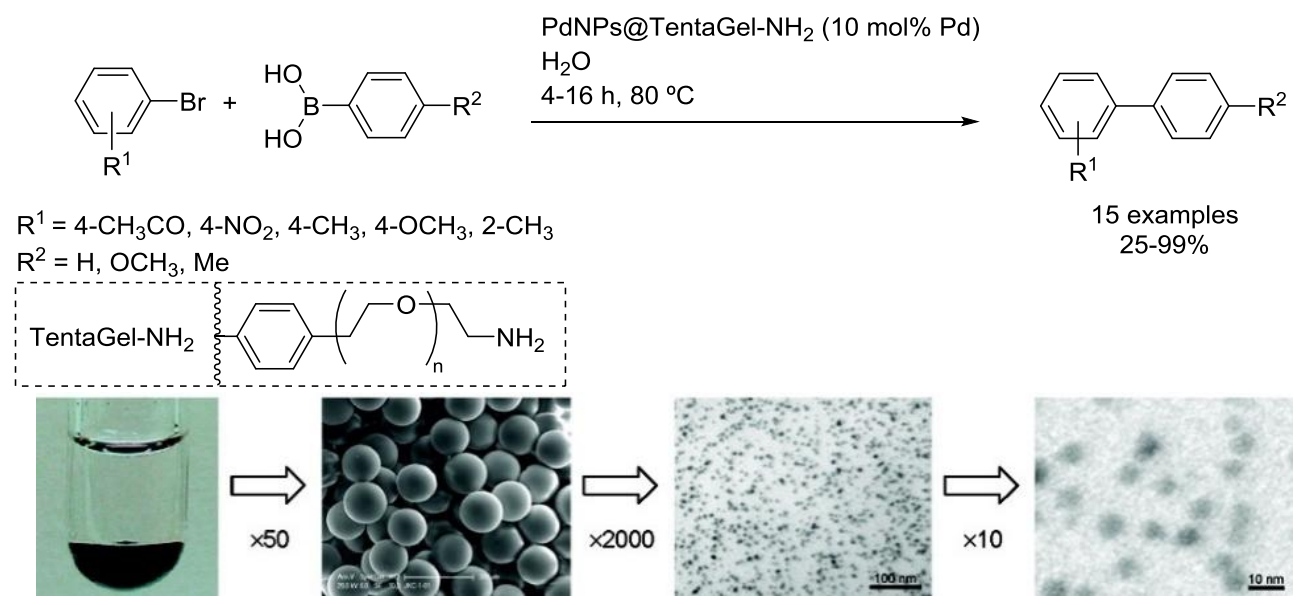

Figure 24. Suzuki-Miyaura cross coupling reaction catalyzed by PdNPs supported in a polystyrene-poly(ethylene glycol) resin. Reprinted with permission. ${ }^{253}$ Copyright (2006) American Chemical Society.

Nájera and co-workers have recently described the use of a mixture of glycerol and urea for the preparation of carbon quantum dots (CQD) on magnetite composites which were used as supports for the immobilization of PdNPs (Pd@CQD@ $\mathrm{Fe}_{3} \mathrm{O}_{4}$, Scheme 24). ${ }^{254}$ The as-prepared catalytic composites catalyzed the Suzuki-Miyaura cross coupling reaction of aryl bromides and boronic acids at low catalyst loadings $(0.3 \mathrm{~mol} \% \mathrm{Pd})$, exhibiting uniform particles of $15-20 \mathrm{~nm}$ in size, but Pd species could not be distinguished from the magnetite cores. ${ }^{254} \mathrm{TEM}$ analysis of the magnetically recycled catalyst after ten runs showed that the structure of the composite was preserved; leaching of $\mathrm{Pd}$ and Fe after ten runs was 16 and $0.2 \%$, respectively; which could 
explain the small decrease in yield observed for the reaction of 4-iodoanisole with phenylboronic after to 10 catalytic runs. ${ }^{254}$

Scheme 24. Palladium supported on glycerol-derived carbon quantum dots and magnetite composites. Reproduced with permission from John Wiley and Sons ${ }^{254}$ license number 4558650798931.

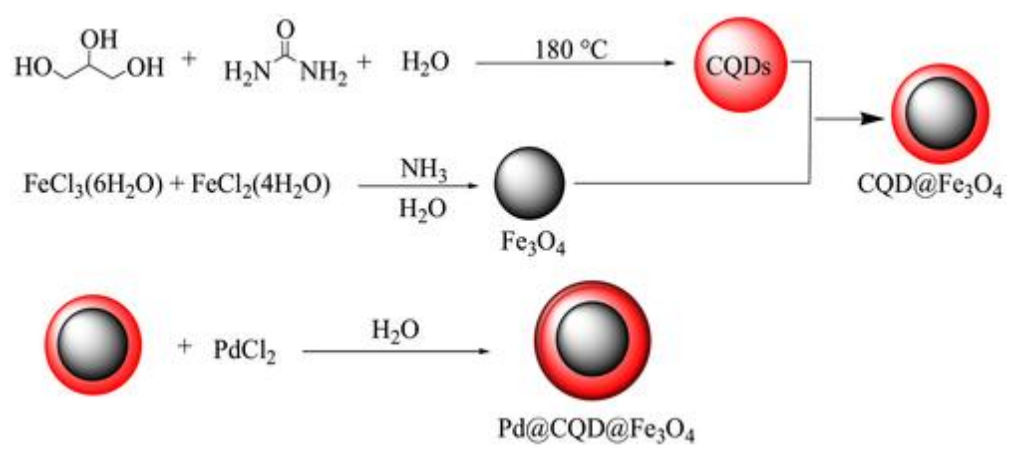

Bathula and co-workers employed Pd nanocatalysts from fenugreek tea for the Suzuki-Miyaura cross-coupling reaction between bromobenzene and phenyl boronic acid at a low catalyst loading $(0.5 \mathrm{~mol} \% \mathrm{Pd})$ in a solvent mixture of polyethylene glycols and water, affording the corresponding 1,1'-biphenyl in $96 \%$ yield. ${ }^{255}$ Many literature reports on the Suzuki-Miyaura cross-coupling feature benchmark reactions between aryl halides (mostly bromide and iodide) and phenyl boronic acid or its ester derivatives to give the symmetric 1,1'-biphenyl product. Despite its large utilization, these benchmark substrates are not well suited to study the crosscoupling reaction as competing homo-coupling reaction manifolds could also be operative and lead to the same product. Thus, building blocks with different substitution patterns should be employed for accurate studies of $\mathrm{C}-\mathrm{C}$ cross-coupling reaction and parent homo-coupling reactions. 
The $\operatorname{Pd}(0)$-catalyzed C-C cross-coupling reactions described by Moumen and Ouaskit used PdNPs immobilized in an aqueous emulsion of copolymers containing poly(vinyl acetate-vinyl versatate) and EG. Their liquid-phase immobilized catalytic system exhibited excellent chemoselectivity and high conversions towards Suzuki, Heck and Sonogashira reactions at catalyst loadings ranging from 1 to $2 \mathrm{~mol} \% \mathrm{Pd}^{256}$

As described by Zhang and co-workers, the immobilization of PdNPs into micellar triazolecontaining dendrimers featuring polyethylene glycol spacers has proven an excellent strategy towards catalyst heterogenization for the Suzuki-Miyaura reaction in water (Scheme 25). ${ }^{257}$ The mild reaction conditions required to work with this catalytic system such as room temperature, low catalyst loadings ( $0.5 \%$ or less), combined to the ease of catalyst recovery after each reaction cycle, and the negligible activity decrease towards catalyst recycling and reuse enable sustainable applications. ${ }^{257}$

Scheme 25. Heterogenized PdNPs on triazole- containing cross- linked polyol micelles. Reproduced with permission from John Wiley and Sons, ${ }^{257}$ license number 4558650609114. 

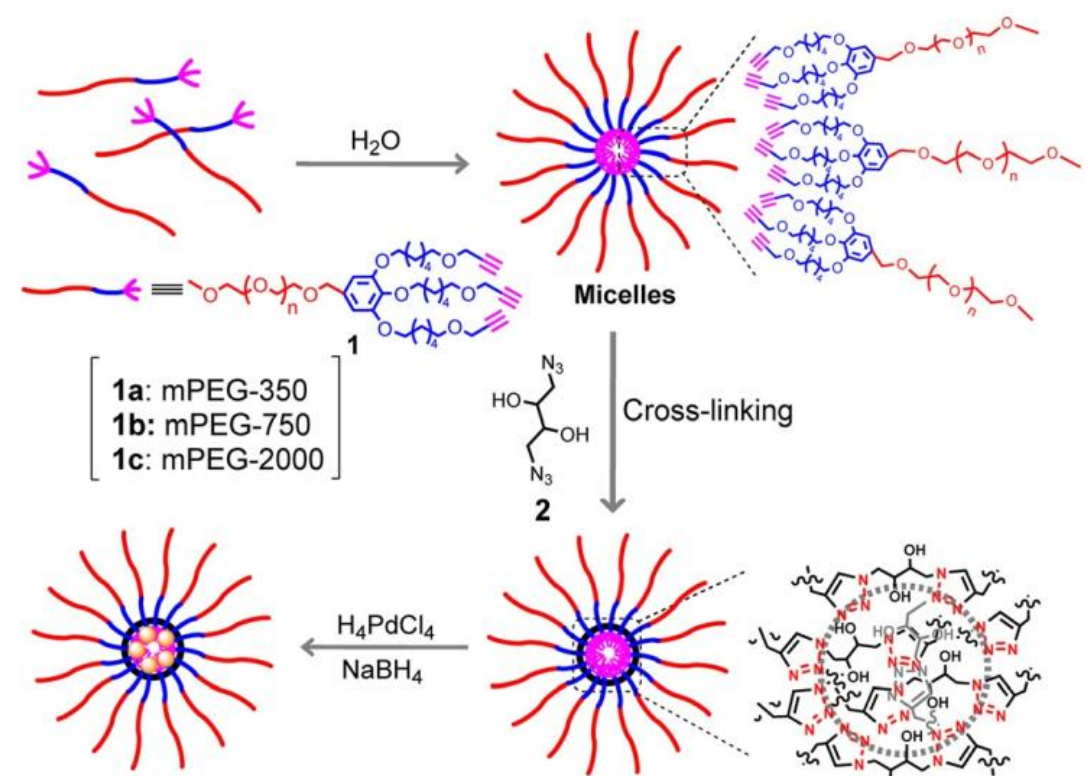

Pd@triazole-DCCMs

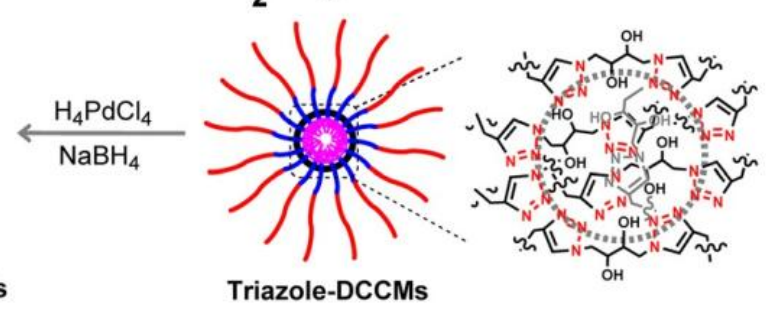

Other types of polymeric matrixes containing cross-linked triarylphosphine moieties, imidazolium ionic liquids and polyethylene glycol have been prepared by Doherty, Knight and co-workers for the immobilization of highly dispersed PdNPs of $<2.5 \mathrm{~nm}$ in average diameter size (Figure 25). ${ }^{258}$ A turnover frequency of $16300 \mathrm{~h}^{-1}$ at room temperature is one of the highest for Pd nanocatalyzed Suzuki-Miyaura cross-coupling of 4-bromoacetophenone and phenylboronic to date at catalyst loading as low as $0.05 \mathrm{~mol} \% \mathrm{Pd}^{258}$ 


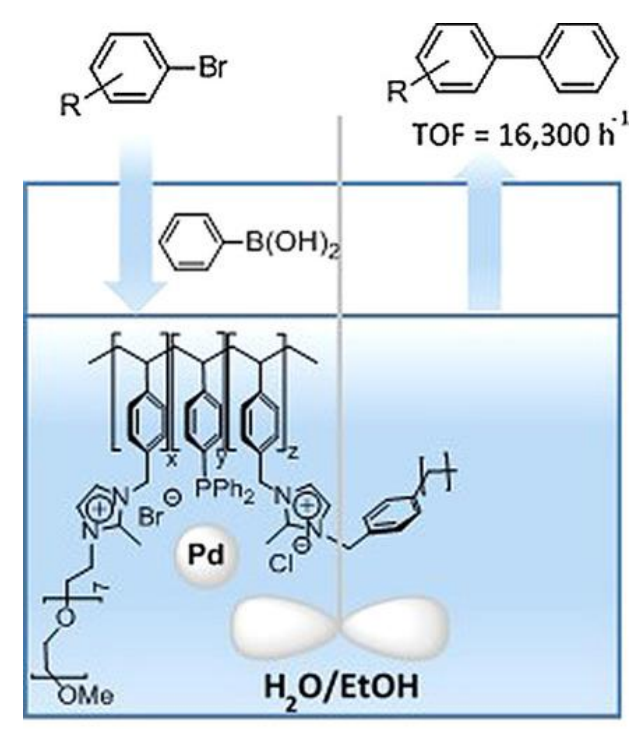

Figure 25. Pd nanoparticle-supported on polymeric matrixes featuring cross-linked triarylphosphine moieties, imidazolium ionic liquids and polyethylene glycol. Reproduced with permission from John Wiley and Sons, ${ }^{258}$ license number 4558650410987.

Carbon-supported PdNPs (2.0-2.5 nm average mean size) have been prepared by decomposition of $\mathrm{Pd}_{2}(\mathrm{dba})_{3}$ under $\mathrm{H}_{2}$ atmosphere in the presence of spherical mesoporous carbon beads (average particle sizes ranging in the $0.5-1.0 \mathrm{~mm}$ range) by García-Suárez and Philippot. ${ }^{259}$ The nanocatalysts exhibited excellent activities at catalyst loadings ranging from 0.4 to $1.5 \mathrm{~mol} \% \mathrm{Pd}$ towards Suzuki-Miyaura cross-coupling in PEG/water mixtures under conventional heating without any loss of activity up to 10 recyclings.

Furthermore, Nikoorazm and co-workers reported a grafted palladium $S$-benzylisothiourea complex on MCM-41 (Pd-SBT@MCM-41) reduced with $\mathrm{NaBH}_{4}$ to generate PdNPs of $4 \pm 1 \mathrm{~nm}$ in average diameter. The as-prepared material catalyzed Suzuki Miyaura cross-coupling reactions of aryl halides and phenylboronic acid in PEG in high efficiency at $1.8 \mathrm{~mol} \% \mathrm{Pd}$ 
catalyst loading. Catalyst recovery was easily performed by centrifugation and it remained active up to six consecutive runs without loss in activity. ${ }^{260}$

An heterogenization method described by Naghipour and co-workers consists of a pyridinecarboxaldehyde-derivatized magnetite-containing chitosan as support for $\mathrm{Pd}$ nanocatalysts. The as-prepared catalytic material featuring crystallites of $31 \mathrm{~nm}$ average size (as determined by PXRD) was highly efficient towards Suzuki-Miyaura cross-coupling (at 1 mol\% Pd catalyst loading) and it was magnetically-recovered with no significant losses in catalytic activity up to 5 runs. ${ }^{261}$ In terms of substrate scope, aryl halides were efficiently coupled to phenylboronic acid using $\mathrm{K}_{2} \mathrm{CO}_{3}$ in PEG to yield the corresponding unsymmetrical bis-aryls in $40-99 \%$ yields. $^{261}$

An alternative Pd nanoparticle immobilization strategy using a polyol-containing co-polymer of styrene and 3-(2,3-dihydroxypropyl)-1-vinylimidazolium chloride as support have been reported by Song and co-workers. ${ }^{262}$ The as-prepared material of co-polymer dispersed nanoparticles (4 $\mathrm{nm}$ average size) catalyzed Suzuki-Miyaura cross-coupling with particularly low catalyst loadings $(0.05 \mathrm{~mol} \% \mathrm{Pd})$ in water-ethanol solvent mixtures at mild temperature $\left(70{ }^{\circ} \mathrm{C}\right)$. Despite the authors highlighted in several occasions that this $\operatorname{Pd}(0)$-catalyzed cross-coupling reaction is run under air atmosphere, they do not further comment on potentially competing homo-coupling reaction manifolds such as the $\mathrm{O}_{2}$-mediated homo-coupling reaction of boronic acids.

\section{6. $\mathrm{C}\left(\mathrm{sp}^{2}\right)-\mathrm{C}\left(\mathrm{sp}^{2}\right)-\mathrm{C}\left(\mathrm{sp}^{2}\right)$ carbonylative Suzuki cross-coupling}

The migratory insertion of carbon monoxide to palladium is an elementary step that can efficiently intercept the classical reaction manifold of the Suzuki cross coupling reaction towards 
the synthesis of arylketone derivatives. ${ }^{263}$ In particular, Han and co-workers have described a highly selective carbonylative reaction using in situ generated PdNPs (of $2.6 \mathrm{~nm}$ in average particle size) by decomposition of $\mathrm{Pd}(\mathrm{OAc})_{2}$ in $\mathrm{PEG}^{264}$ This nanocatalytic system converted electron-donating or electron-withdrawing aryl iodides with arylboronic acids into the corresponding diaryl ketones at $2 \mathrm{~mol} \% \mathrm{Pd}$ catalyst loading, room temperature and under ambient pressure of $\mathrm{CO}$ employing pivalic acid as an additive to effectively suppress the corresponding Suzuki-Miyaura coupling (Scheme 26) at gram-scale. According to the authors, this transformation operates under a homogeneous regime as determined by the total inhibition of the reaction in the presence of equimolar amounts of $\mathrm{CS}_{2}$ and negative $\mathrm{Hg}$ poisoning tests. ${ }^{264}$

Scheme 26. Pd-catalyzed carbonylative Suzuki reactions of aryl iodides and arylboronic acids. ${ }^{264}$

4.7. $\mathrm{C}\left(\mathrm{sp}^{2}\right)-\mathrm{C}\left(\mathrm{sp}^{2}\right)$ Ullmann-type homo-coupling.

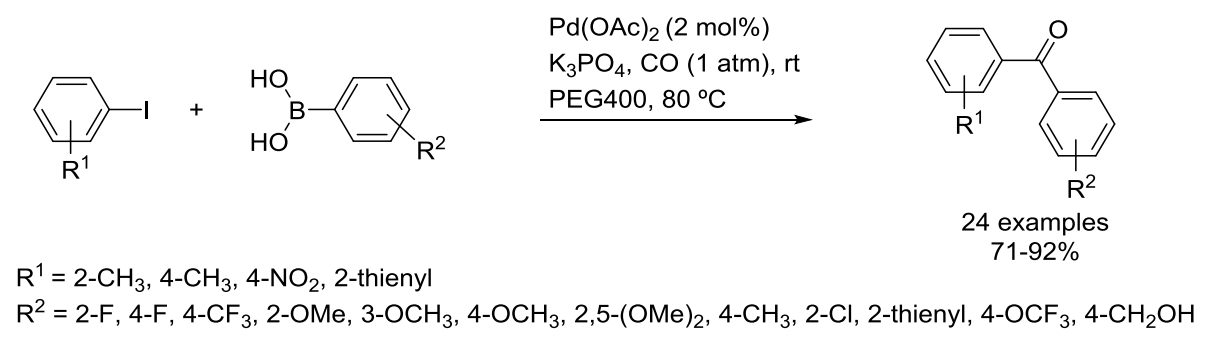

Beyond the aerobic homo-coupling reaction of boronic acid derivatives via the formation of palladium (II) peroxo species under homogenous conditions (Figure 23), nanocatalysts generated in polyol media show enhanced surface-like reactivity which confers them unique potential towards homo-coupling reactions. Thus, PdNPs of $4 \pm 1 \mathrm{~nm}$ average size synthesized from $\mathrm{H}_{2}\left[\mathrm{PdCl}_{4}\right]$ reduction with $\mathrm{PEG}$ in the presence of a polyethylene imine polymer $(\mathrm{Mw}=25000)$ under sonication at room temperature showed almost quantitative conversion of aryl bromides 
and high yields towards the synthesis of symmetric bis-aryls via homo-coupling reaction at $90{ }^{\circ} \mathrm{C}$ in a solvent mixture of EG - ethanol (1:2) at a catalyst loading below $1 \mathrm{~mol} \% \mathrm{Pd} .{ }^{265}$ Different mechanistic approaches have been proposed for the synthesis of bis-aryls by homo-coupling reaction. For instance, Mandal and co-workers reported a homogeneous catalytic cycle encompassing one oxidative addition of an aryl halide to a $\operatorname{Pd}(0)$ metal center which delivers an arylpalladium (II) species. ${ }^{265}$ Although, the following elementary step involving the activation of a second molecule of aryl halide, leading to the formation of diarylpalladium (II) species with a concomitant loss of the halide salt, seems rather unrealistic for these reviewers. ${ }^{265}$

Our group has recently described a highly selective nanocatalyst for C-C homo-coupling reactions prepared from $\mathrm{Pd}(\mathrm{OAc})_{2}$ and cinchona-based alkaloids (quinidine and cinchonidine) under $\mathrm{H}_{2}$ ( 3 bar) in neat glycerol. ${ }^{235}$ The metal surface and stabilizer coordination mode via $\pi-\pi$ interactions is key for promoting this catalytic pathway. The mechanistic studies derived from this study with an analogous preformed zero-valent Pd nanocatalysts stabilized with PVP in neat glycerol led to the identification of a surface-mediated radical manifold based on single-electron transfer processes (Figure 26), with the experimental identification of the aryl bromide radical anion by electron paramagnetic resonance (EPR). Thus, aryl bromides homocouplings proceeded efficiently to produce the corresponding bis-aryls in moderate to excellent yields and using relatively low catalyst loadings ( $1 \mathrm{~mol} \% \mathrm{Pd}){ }^{266}$ The as-prepared nanocatalysts from different $\mathrm{Pd}$ salt precursors were quite sensitive towards surface poisoning by halides, notably iodide hampered the reactivity. The halide/Pd ratio was determined by X-ray fluorescence analysis. ${ }^{266}$ This work sheds light on the crucial role of $\operatorname{Pd}(0)$ nanocatalyst surfaces towards enabling the activation of aryl halides involving the Fermi level electrons. 


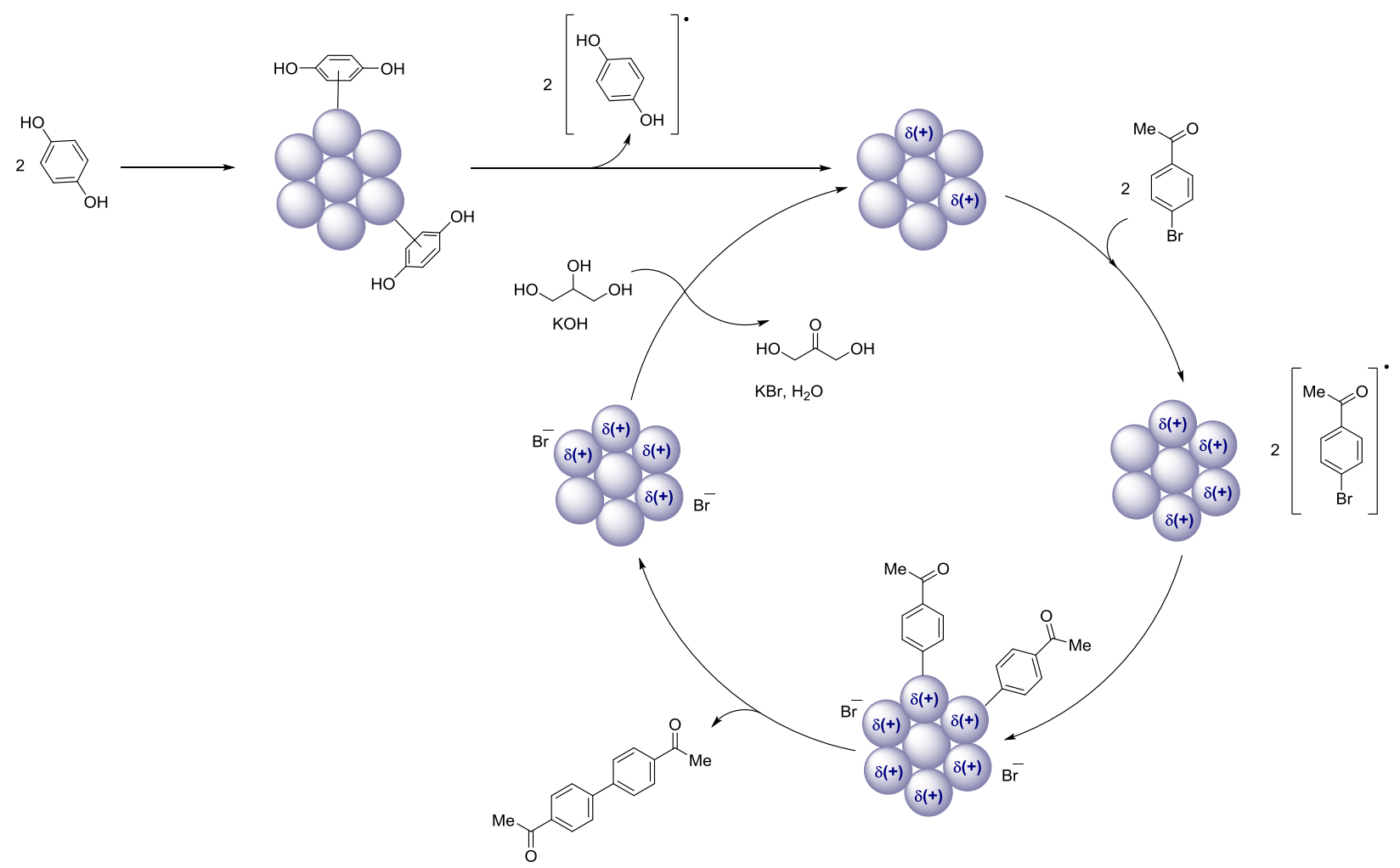

Figure 26. Proposed radical homo-coupling reaction mechanism promoted by PdNPs involving neighboring metal centers. ${ }^{266}$

4.8. Miscellaneous: $\mathrm{Si}-\mathrm{N}$ and $\mathrm{C}-\mathrm{N}$ amination reactions.

The previously Pd nanocatalyst systems described by our group prepared with cinchona-based alkaloids (cinchonidine, quinidine) as stabilizers in neat glycerol also exhibited high performance in catalyzing Michael conjugate additions at low catalyst loadings ( 1 and $0.1 \mathrm{~mol} \% \mathrm{Pd}$ ), revealing the importance of large surface areas in favoring this reactivity in an heterogeneouslike manifold. ${ }^{235}$

Alternative highly efficient processes, such as the synthesis of 5 -substituted $1 H$-tetrazoles via a $[2+3]$ cycloaddition of arylnitriles with sodium azide has been described by Nikoorazm and co- 
workers with a reusable Pd nanocatalyst supported on functionalized MCM-41 in PEG at 5.4 mol\% Pd catalyst loading. ${ }^{260}$

Besides, a surface dehydrogenative coupling of organosilanes leveraged by PdNPs for the formation of Si-N bond has recently been described by Wang and co-workers This nanocatalytic system outsmarts existing catalysts in terms of durability characteristics and higher level of efficiency. In a broader context, these results illustrate powerful nanocatalytic approaches to maximize the performance of palladium catalysis in the quest of green and sustainable processes required for future industrial applications.

\section{Conclusions and Outlook}

In this review, we highlight the paramount relevance of polyols in the synthesis of metal-based nanoparticles, in particular through the palladium case; in most reported examples, the reactions are carried out in the presence of water, but also in neat polyol. One of the unrivalled advantages of polyols, and alcohols in general, is their versatility, working as multi-task reaction components, i.e. reductant of metallic precursors, solvent, and sometimes even as stabilizer. But this latter function towards precluding MNPs agglomeration is mainly reserved to polyol derivatives, such as those polyols containing ethers handles in one or several hydroxyl groups of the polyol, especially the ones featuring long carbon chains. Furthermore, polyols promote the dispersion of the formed MNPs mainly due to their hydrogen-bonded supramolecular structure. Polyols have been classically used for the synthesis of high electronegativity metals (such as Ag, $\mathrm{Au}, \mathrm{Pd}, \mathrm{Pt} . .$.$) , mainly due to the ease of reduction of these metals. Notwithstanding, polyols are$ appropriate solvents for $3 \mathrm{~d}$ transition metals in combination with stronger reducing agents for the synthesis of zero-valent MNPs given the relative low solubility of molecular oxygen in polyol 
media, avoiding in this case the formation of products coming from the oxidation of the corresponding polyols (aldehydes, ketones). More recently, polyols coming from plant extracts have attracted the attention of some groups for the preparation of well-defined nanoparticles; at present, the main concern is the control of the surface state of these as-prepared nanoparticles, highly dependent on the nature of these bio-polyols as raw materials. In this review, we focus on palladium given its versatility in catalysis towards streamlined organic synthesis, mainly couplings and hydrogenations processes, among others. In addition, its efficiency is proven at angstrom (homogeneous), micrometer (heterogeneous), and nanometer (homogeneous -reservoir of complexes- or heterogeneous -surface reactivity-) scales, being palladium able to stabilize different types of structures with an impact towards reactivity. Due to its robustness, palladium can undergo different type of activation modes (classical heating, microwaves irradiation, ultrasounds). Polyols together with stabilizers, often polymers such as PVP but also ligands such as phosphines, thiols or aromatic nitrogen-based stabilizers, are able to control the size and shape at the nanometric level, with a great potential for applications. Furthermore, PdNPs can be prepared under batch and continuous-flow conditions; this latter approach is in particular important from a mechanistic point of view, permitting the monitoring of PdNPs synthesis, and then the study of the kinetics for the different synthetic steps (reduction of metal precursor, nucleation, growth), which can be crucial for the synthesis of bimetallic nanoparticles. As can be ascertained from this contribution, the methods for preparing PdNPs and in general metal-based nanoparticles using polyols as solvents are largely applied for bottom-up methods, namely decomposition/reduction of metallic precursors to generate in situ "naked" metal atoms that quickly form nuclei, which produce nano-objects under appropriate and well-controlled conditions (temperature, pressure, concentration, stabilizer...). However, top-down methods other 
than electrochemical approaches are rarely described in the literature. Recently, Staszek and coworkers have reported the synthesis of noble metal nanoparticles $(\mathrm{Ag}, \mathrm{Au}, \mathrm{Pd}, \mathrm{Pt})$ by sputtering metal atoms in anhydrous glycerol. ${ }^{267}$ The main concern is related to the vapor pressure of polyols, because sputter technique works under high vacuum $\left(<10^{-7} \mathrm{mmHg}\right)$. However, this limitation can be overcome for high molecular weight polyols. This methodology leads to the synthesis of mono- or polymetallic nanocatalysts precluding the oxidation of the polyol, being an attractive approach for applications; currently, its main restraint is associated to the limited development of sputtering techniques using liquids as substrates.

Curiously and in clear contrast to other media (conventional organic solvents, ionic liquids, water,), PdNPs in polyol have been less exploited. This review describes the use of PdNPs in polyol medium or polyol supported polymeric matrixes as catalysts towards hydrogenations, and C-C cross-coupling (Sonogashira, Hiyama-Denmark, Suzuki-Miyaura, Mizoroki-Heck), Ullmann-type homo-coupling and amination reactions. A critical discussion on the operating reaction mechanisms has been presented, especially for $\mathrm{C}-\mathrm{C}$ couplings with the aim of highlighting the nature of the catalytically active species, thereby the substrate activation modes. In general terms, nanocatalytic systems enable molecular and/or surface reactivity. In polyol medium, in contrast to conventional solvents, more efforts are needed to elucidate the operating reaction regimes ultimately enabling rational catalyst design.

The advantages, in terms of compatibility concerning functional groups and activation modes, presented by nanocatalysts in polyol media envisage their application towards downstreaming sustainable processes, such as $\mathrm{C}-\mathrm{C}$ or $\mathrm{C}$-heteroatom bond formation by $\mathrm{C}-\mathrm{H}$ activation. Progress on flow chemistry applications enables the integration of both tailor-made MNPs syntheses coupled to catalytic transformations, and reaction monitoring. 


\section{AUTHOR INFORMATION}

Corresponding Authors

Daniel PLA pla@lhfa.fr

Montserrat GÓMEZ gomez@chimie.ups-tlse.fr

\section{ORCID}

Isabelle Favier: 0000-0002-0590-0708

Daniel Pla: 0000-0002-8703-8778

Montserrat Gómez: 0000-0003-1211-1333

Notes

The authors declare no competing financial interest

Biographies

Isabelle Favier holds a PhD degree in Chemistry from the University of Nice-Sophia Antipolis (France), obtained in 2001 under the supervision of Dr. E. Duñach, on Bi-catalyzed oxidation reactions and the electro-synthesis of Lewis acids. After a post-doctoral stay (2003-2005) in the group of Prof. G. Muller in the University of Barcelona (Spain) working on the synthesis of chiral polymers as stabilizers for metal nanoparticles, she joined the group of Prof. M. Gómez in Toulouse being involved in metallic nanoparticles confinement into carbon nanotubes. Since 2008, she holds a permanent position as Ingénieur d'Etudes CNRS at the University of Toulouse 3 - Paul Sabatier (France). Her research interests are focused on homogeneous and colloidal metal catalysis. 
Daniel Pla obtained his PhD from the University of Barcelona (Spain) in 2009. He then pursued postdoctoral studies at the Molecular Pharmacology and Chemistry Program at the Memorial Sloan-Kettering Cancer Center in New York (USA), and the Department of Chemistry at the University of Cambridge (UK) as a Marie Curie fellow. In 2014, he joined the Laboratory of Coordination Chemistry (LCC) in Toulouse as a postdoctoral research associate. Since 2016, he holds a CNRS researcher position at the Fundamental and Applied Heterochemistry Laboratory (LHFA), a joint unit from the CNRS and the University of Toulouse 3 - Paul Sabatier and his current research goals focus on metal-based catalysis (complexes, nanoparticles) in nonconventional solvents, and catalyst heterogenization by selective functionalization of supports, albeit with particular interest into mechanistical insights.

Montserrat Gómez received her PhD in 1991 from the University of Barcelona (UB, Spain), in organometallic chemistry and homogeneous catalysis. She carried out a post-doc in the group of Dr. I. Tkatckenko (Toulouse, France, 1992) and sabbatical stays in the teams of Prof. P.W.N.M. van Leeuwen (Amsterdam, 1998) and Dr. B. Chaudret (Toulouse, 2002). In the period 19932004, she occupied a Lecturer position in the UB working on chiral ligands design and enantioselective catalysis. Since 2005, she is Full Professor at the University of Toulouse 3 Paul Sabatier (Toulouse). Her current research work is focused on metal transition-catalyzed processes mainly using non-conventional solvents (ionic liquids, glycerol) and innovative functionalized supports (hydrochars, halloysites), including polymetallic systems and devoting special interest to the mechanistic aspects.

\section{ACKNOWLEDGMENTS}

Financial support from the Centre National de la Recherche Scientifique (CNRS) and the University of Toulouse 3 - Paul Sabatier are gratefully acknowledged. 


\section{ABBREVIATIONS}

acac acetyl acetonate

AES Atomic Emission Spectroscopy

AIBN Azobisisobutyronitrile

BMNPs Bimetallic nanoparticles

COD 1,5-Cyclooctadiene

CTAB cetyltrimethylammonium bromide

dba dibenzylidenacetone

DEG Diethylene glycol

DFT Density Functional Theory

DMF $N, N$-Dimethylformamide

EDS Energy-Dispersive X-ray Spectroscopy

EG Ethylene glycol

EXAFS Extended X-ray Absorption Fine Structure

FE-TEM Field-Emission Transmission Electron Microscopy

Gly Glycerol

HAADF High-Angle Annular Disk Field

HMBC Heteronuclear Multiple Bond Correlation

HRTEM High Resolution Transmission Electron Microscopy

ICP Inductively Coupled Plasma

ma maleic anhydride

MCM-41 Mobil Composition of Matter No. 41

MNPs Metallic Nanoparticles

NAPXPS Near-Ambient Pressure X-Ray Photoelectron Spectroscopy

nbd norbornadiene

NMR Nuclear Magnetic Resonance

NPs Nanoparticles 
PBS Phosphate Buffered Saline

PCA Polycitric acid

PEG Polyethylene glycol

PTA 1,3,5-Triaza-7-phosphaadamantane

PVP Poly(vinyl)pyrrolidone

PVPy Poly(4-vinylpyridine)

rt room temperature

SAXS Small Angle X-ray Scattering

SDS Sodium dodecyl sulfate

SERS Surface-Enhanced Raman Scattering

STEM Scanning Transmission Electron Microscopy

SQUID Superconducting Quantum Interference Device

TEG Tetraethylene glycol

TEM Transmission electron microscopy

TMEDA tetramethylethylendiamine

TPPTS trisodium 3,3',3-phosphinetriyltribenzenesulfonate

TrEG triethylene glycol

TTAB trimethyl(tetradecyl)ammonium bromide

XPS X-Ray Photoelectron Spectroscopy

XRD X-Ray Diffraction

\section{REFERENCES}

(1) Tsuji, J. Palladium in Organic Synthesis; Springer Berlin Heidelberg, 2005.

(2) Tsuji, J. Palladium Reagents and Catalysts: New Perspectives for the 21st Century; Wiley: West Sussex, 2004.

(3) Johansson Seechurn, C. C. C.; Kitching, M. O.; Colacot, T. J.; Snieckus, V. PalladiumCatalyzed Cross-Coupling: A Historical Contextual Perspective to the 2010 Nobel Prize. Angew. Chem., Int. Ed. 2012, 51, 5062-5085. 
(4) Liu, X.; Astruc, D. Development of the Applications of Palladium on Charcoal in Organic Synthesis. Adv. Synth. Catal. 2018, 360, 3426-3459.

(5) Monguchi, Y.; Ichikawa, T.; Sajiki, H. Recent Development of Palladium-Supported Catalysts for Chemoselective Hydrogenation. Chem. Pharm. Bull. 2017, 65, 2-9.

(6) Molnár, Á. Efficient, Selective, and Recyclable Palladium Catalysts in Carbon-Carbon Coupling Reactions. Chem. Rev. 2011, 111, 2251-2320.

(7) Bönnemann, H.; Brijoux, W.; Brinkmann, R.; Joußen, T.; Korall, B.; Dinjus, E. Formation of Colloidal Transition Metals in Organic Phases and Their Application in Catalysis. Angew. Chem., Int. Ed. 1991, 30, 1312-1314.

(8) Reetz, M. T.; Lohmer, G. Propylene Carbonate Stabilized Nanostructured Palladium Clusters as Catalysts in Heck Reactions. Chem. Commun. 1996, 1921-1922.

(9) Schmid, G. Nanoparticles: From Theory to Application; Wiley, 2011.

(10) Serp, P.; Philippot, K.; Somorjai, G. A.; Chaudret, B. Nanomaterials in Catalysis; Wiley, 2012.

(11) Martínez-Prieto, L. M.; Chaudret, B. Organometallic Ruthenium Nanoparticles: Synthesis, Surface Chemistry, and Insights into Ligand Coordination. Acc. Chem. Res. 2018, 51, 376-384.

(12) Adil, S. F.; Assal, M. E.; Khan, M.; Al-Warthan, A.; Siddiqui, M. R. H.; Liz-Marzán, L. M. Biogenic Synthesis of Metallic Nanoparticles and Prospects Toward Green Chemistry. Dalton Trans. 2015, 44, 9709-9717.

(13) Ghosh Chaudhuri, R.; Paria, S. Core/Shell Nanoparticles: Classes, Properties, Synthesis Mechanisms, Characterization, and Applications. Chem. Rev. 2012, 112, 2373-2433.

(14) Zhou, B.; Hermans, S.; Somorjai, G. A. Nanotechnology in Catalysis; Kluwer/Plenum: New York, 2004.

(15) An, K.; Alayoglu, S.; Ewers, T.; Somorjai, G. A. Colloid Chemistry of Nanocatalysts: A Molecular View. J. Colloid Interface Sci. 2012, 373, 1-13.

(16) Metal Nanoclusters in Catalysis and Materials Science: The Issue of Size Control; Elsevier Science: Amsterdam, 2011.

(17) Astruc, D. Nanoparticles and Catalysis; Wiley, 2008.

(18) Heiz, U.; Landman, U. Nanocatalysis; Springer: Berlin, 2007.

(19) Torimoto, T.; Kameyana, T.; Kuwabata, S. Top-down Synthesis Methods for Nanoscale Catalysts; In Nanocatalysis in Ionic Liquids; Prechtl, M. H. G., Ed.; Wiley- VCH: Weinheim, 2017 Ch. 9, pp 171-205.

(20) Roucoux, A.; Schulz, J.; Patin, H. Reduced Transition Metal Colloids: a Novel Family of Reusable Catalysts? Chem. Rev. 2002, 102, 3757-3778.

(21) Bulut, S.; Fei, Z.; Siankevich, S.; Zhang, J.; Yan, N.; Dyson, P. J. Aqueous-phase Hydrogenation of Alkenes and Arenes: The Growing Role of Nanoscale Catalysts. Catal. Today 2015, 247, 96-103.

(22) San, K.; Shon, Y.-S. Synthesis of Alkanethiolate-capped Metal Nanoparticles Using Alkyl Thiosulfate Ligand Precursors: a Method to Generate Promising Reagents for Selective Catalysis. Nanomaterials 2018, 8, 346.

(23) In Nanocatalysis in Ionic Liquids; Prechtl, M. H. G., Ed.; Wiley- VCH: Weinheim, 2017 $\mathrm{pp}$

(24) Dahl, J. A.; Maddux, B. L. S.; Hutchison, J. E. Toward Greener Nanosynthesis. Chem. Rev. 2007, 107, 2228-2269. 
(25) Patete, J. M.; Peng, X.; Koenigsmann, C.; Xu, Y.; Karn, B.; Wong, S. S. Viable Methodologies for the Synthesis of High-quality Nanostructures. Green Chem. 2011, 13, 482-519.

(26) Zhang, Y.; Erkey, C. Preparation of Supported Metallic Nanoparticles Using Supercritical Fluids: a Review. J. Supercrit. Fluids 2006, 38, 252-267.

(27) Fiévet, F.; Ammar-Merah, S.; Brayner, R.; Chau, F.; Giraud, M.; Mammeri, F.; Peron, J.; Piquemal, J. Y.; Sicard, L.; Viau, G. The Polyol Process: a Unique Method for Easy Access to Metal Nanoparticles with Tailored Sizes, Shapes and Compositions. Chem. Soc. Rev. 2018, 47, 5187-5233.

(28) Favier, I.; Pla, D.; Gómez, M. Metal-based Nanoparticles Dispersed in Glycerol: an Efficient Approach for Catalysis. Catal. Today 2018, 310, 98-106.

(29) Chahdoura, F.; Favier, I.; Gómez, M. Glycerol as Suitable Solvent for the Synthesis of Metallic Species and Catalysis. Chem. Eur. J. 2014, 20, 10884-10893.

(30) Fievet, F.; Lagier, J. P.; Blin, B.; Beaudoin, B.; Figlarz, M. Homogeneous and Heterogeneous Nucleations in the Polyol Process for the Preparation of Micron and Submicron Size Metal Particles. Solid State Ion. 1989, 32-33, 198-205.

(31) Fievet, F.; Lagier, J. P.; Figlarz, M. Preparing Monodisperse Metal Powders in Micrometer and Submicrometer Sizes by the Polyol Process. MRS Bull. 2013, 14, 29-34.

(36) Gladysz John, A. Recoverable Catalysts. Ultimate Goals, Criteria of Evaluation, and the Green Chemistry Interface. Pure Appl. Chem. 2001, 73, 1319-1324.

(38) Phan, N. T. S.; Van Der Sluys, M.; Jones, C. W. On the Nature of the Active Species in Palladium Catalyzed Mizoroki-Heck and Suzuki-Miyaura Couplings - Homogeneous or Heterogeneous Catalysis, A Critical Review. Adv. Synth. Catal. 2006, 348, 609-679.

(40) Arora, S.; Singla, M. L.; Kapoor, P. Evidence for Monoalkoxide Species on the Surface of Palladium Nanoparticles Synthesized in Ethylene Glycol. Mater. Chem. Phys. 2009, 114, 107-112.

(41) Xiong, Y.; Chen, J.; Wiley, B.; Xia, Y.; Aloni, S.; Yin, Y. Understanding the Role of Oxidative Etching in the Polyol Synthesis of Pd Nanoparticles with Uniform Shape and Size. J. Am. Chem. Soc. 2005, 127, 7332-7333. 
(42) Baletto, F.; Ferrando, R. Structural Properties of Nanoclusters: Energetic, Thermodynamic, and Kinetic Effects. Rev. Mod. Phys. 2005, 77, 371-423.

(43) Lv, T.; Wang, Y.; Choi, S.-I.; Chi, M.; Tao, J.; Pan, L.; Huang, C. Z.; Zhu, Y.; Xia, Y. Controlled Synthesis of Nanosized Palladium Icosahedra and Their Catalytic Activity Towards Formic-Acid Oxidation. ChemSusChem 2013, 6, 1923-1930.

(44) Huang, H.; Wang, Y.; Ruditskiy, A.; Peng, H.-C.; Zhao, X.; Zhang, L.; Liu, J.; Ye, Z.; Xia, Y. Polyol Syntheses of Palladium Decahedra and Icosahedra as Pure Samples by Maneuvering the Reaction Kinetics with Additives. ACS Nano 2014, 8, 7041-7050.

(45) Wang, Y.; Peng, H.-C.; Liu, J.; Huang, C. Z.; Xia, Y. Use of Reduction Rate as a Quantitative Knob for Controlling the Twin Structure and Shape of Palladium Nanocrystals. Nano Lett. 2015, 15, 1445-1450.

(46) Li, C.; Sato, R.; Kanehara, M.; Zeng, H.; Bando, Y.; Teranishi, T. Controllable Polyol Synthesis of Uniform Palladium Icosahedra: Effect of Twinned Structure on Deformation of Crystalline Lattices. Angew. Chem., Int. Ed. 2009, 48, 6883-6887.

(47) Long, N. V.; Hayakawa, T.; Matsubara, T.; Chien, N. D.; Ohtaki, M.; Nogami, M. Controlled Synthesis and Properties of Palladium Nanoparticles. J. Exp. Nanosci. 2012, 7, 426-439.

(48) Long, N. V.; Nguyen, D. C.; Hirata, H.; Ohtaki, M.; Hayakawa, T.; Nogami, M. Chemical Synthesis and Characterization of Palladium Nanoparticles. Adv. Nat. Sci. Nanosci. 2010, 1, 035012 (5 pp).

(49) Hei, H.; Wang, R.; Liu, X.; He, L.; Zhang, G. Controlled Synthesis and Characterization of Nobel Metal Nanoparticles. Soft Nanosci. Lett. 2012, 2, 34-40.

(50) Feng, C.; Guo, L.; Shen, Z.; Gong, J.; Li, X.-Y.; Liu, C.; Yang, S. Synthesis of Short Palladium Nanoparticle Chains and their Application in catalysis. Solid State Sci. 2008, 10, 1327-1332.

(51) Schiavo, L.; Aversa, L.; Tatti, R.; Verucchi, R.; Carotenuto, G. Structural Characterizations of Palladium Clusters Prepared by Polyol Reduction of [PdC14]2- Ions. J. Anal. Methods Chem. 2016, 2016, 9073594 (6 pp).

(52) Dahal, N.; García, S.; Zhou, J.; Humphrey, S. M. Beneficial Effects of MicrowaveAssisted Heating versus Conventional Heating in Noble Metal Nanoparticle Synthesis. ACS Nano 2012, 6, 9433-9446.

(53) Yu, Y.; Zhao, Y.; Huang, T.; Liu, H. Microwave-assisted Synthesis of Palladium Nanocubes and Nanobars. Mat. Res. Bull. 2010, 45, 159-164.

(54) Xiao, C.; Ding, H.; Shen, C.; Yang, T.; Hui, C.; Gao, H. J. Shape-Controlled Synthesis of Palladium Nanorods and Their Magnetic Properties. J. Phys. Chem. C 2009, 113, 1346613469.

(55) Xiong, Y.; McLellan, J. M.; Chen, J.; Yin, Y.; Li, Z.-Y.; Xia, Y. Kinetically Controlled Synthesis of Triangular and Hexagonal Nanoplates of Palladium and Their SPR/SERS Properties. J. Am. Chem. Soc. 2005, 127, 17118-17127.

(56) Harada, M.; Cong, C. Microwave-Assisted Polyol Synthesis of Polymer-Protected Monometallic Nanoparticles Prepared in Batch and Continuous-Flow Processing. Ind. Eng. Chem. Res. 2016, 55, 5634-5643.

(57) Okitsu, K.; Bandow, H.; Maeda, Y.; Nagata, Y. Sonochemical Preparation of Ultrafine Palladium Particles. Chem. Mater. 1996, 8, 315-317. 
(58) Nemamcha, A.; Rehspringer, J.-L.; Khatmi, D. Synthesis of Palladium Nanoparticles by Sonochemical Reduction of Palladium(II) Nitrate in Aqueous Solution. J. Phys. Chem. B 2006, 110, 383-387.

(59) Declerck, V.; Colacino, E.; Bantreil, X.; Martinez, J.; Lamaty, F. Poly(ethylene glycol) as Reaction Medium for Mild Mizoroki-Heck Reaction in a Ball-mill. Chem. Commun. 2012, 48, 11778-11780.

(60) Pagliaro, M.; Rossi, M. The Future of Glycerol; The Royal Society of Chemistry, 2010.

(61) García, J. I.; García-Marín, H.; Mayoral, J. A.; Pérez, P. Quantitative Structure-property Relationships Prediction of Some Physico-chemical Properties of Glycerol Based Solvents. Green Chem. 2013, 15, 2283-2293.

(62) Corma, A.; Iborra, S.; Velty, A. Chemical Routes for the Transformation of Biomass into Chemicals. Chem. Rev. 2007, 107, 2411-2502.

(63) Gu, Y.; Jérôme, F. Glycerol as a Sustainable Solvent for Green Chemistry. Green Chem. 2010, 12, 1127-1138.

(64) Díaz-Álvarez, A. E.; Francos, J.; Lastra-Barreira, B.; Crochet, P.; Cadierno, V. Glycerol and Derived Solvents: New Sustainable Reaction Media for Organic Synthesis. Chem. Commun. 2011, 47, 6208-6227.

(65) Puvvada, S.; Baral, S.; Chow, G. M.; Qadri, S. B.; Ratna, B. R. Synthesis of Palladium Metal Nanoparticles in the Bicontinuous Cubic Phase of Glycerol Monooleate. J. Am. Chem. Soc. 1994, 116, 2135-2136.

(66) Lee, Y.-W.; Han, S.-B.; Ko, A. R.; Kim, H.-S.; Park, K.-W. Glycerol-mediated Synthesis of Pd Nanostructures with Dominant $\{111\}$ Facets for Enhanced Electrocatalytic Activity. Catal. Commun. 2011, 15, 137-140.

(67) Nirmala Grace, A.; Pandian, K. One pot Synthesis of Polymer Protected Pt, Pd, Ag and $\mathrm{Ru}$ Nanoparticles and Nanoprisms under Reflux and Microwave Mode of Heating in Glycerol - a Comparative Study. Mater. Chem. Phys. 2007, 104, 191-198.

(68) Kou, J.; Bennett-Stamper, C.; Varma, R. S. Green Synthesis of Noble Nanometals (Au, $\mathrm{Pt}, \mathrm{Pd}$ ) Using Glycerol under Microwave Irradiation Conditions. ACS Sustainable Chem. Eng. 2013, 1, 810-816.

(69) Clergeaud, G.; Genc, R.; Ortiz, M.; O'Sullivan, C. K. Liposomal Nanoreactors for the Synthesis of Monodisperse Palladium Nanoparticles Using Glycerol. Langmuir 2013, 29, 15405-15413.

(70) Chen, H.; Wei, G.; Ispas, A.; Hickey, S. G.; Eychmüller, A. Synthesis of Palladium Nanoparticles and Their Applications for Surface-Enhanced Raman Scattering and Electrocatalysis. J. Phys. Chem. C 2010, 114, 21976-21981.

(71) Reina, A.; Favier, I.; Pradel, C.; Gómez, M. Stable Zero-Valent Nickel Nanoparticles in Glycerol: Synthesis and Applications in Selective Hydrogenations. Adv. Synth. Catal. 2018, 360, 3544-3552.

(72) Chahdoura, F.; Pradel, C.; Gómez, M. Copper(I) Oxide Nanoparticles in Glycerol: A Convenient Catalyst for Cross-Coupling and Azide-Alkyne Cycloaddition Processes. ChemCatChem 2014, 6, 2929-2936.

(73) Dang-Bao, T.; Pradel, C.; Favier, I.; Gómez, M. Making Copper(0) Nanoparticles in Glycerol: A Straightforward Synthesis for a Multipurpose Catalyst. Adv. Synth. Catal. 2017, 359, 2832-2846. 
(74) Chahdoura, F.; Pradel, C.; Gómez, M. Palladium Nanoparticles in Glycerol: A Versatile Catalytic System for C-X Bond Formation and Hydrogenation Processes. Adv. Synth. Catal. 2013, 355, 3648-3660.

(75) Gómez, M.; Teuma, E.; Favier, I.; Chahdoura, F. Metal Nano-catalysts in Glycerol and Applications in Organic Synthesis. PCT Int. Appl. 2014, WO2014096732 A2014096731 2020140626.

(76) Chahdoura, F.; Favier, I.; Pradel, C.; Mallet-Ladeira, S.; Gómez, M. Palladium Nanoparticles Stabilised by PTA Derivatives in Glycerol: Synthesis and Catalysis in a Green Wet Phase. Catal. Commun. 2015, 63, 47-51.

(77) Favier, I.; Massou, S.; Teuma, E.; Philippot, K.; Chaudret, B.; Gómez, M. A New and Specific Mode of Stabilization of Metallic Nanoparticles. Chem. Commun. 2008, 32963298.

(78) Kohlmann, O.; Steinmetz, W. E.; Mao, X.-A.; Wuelfing, W. P.; Templeton, A. C.; Murray, R. W.; Johnson, C. S. NMR Diffusion, Relaxation, and Spectroscopic Studies of Water Soluble, Monolayer-Protected Gold Nanoclusters. J. Phys. Chem. B 2001, 105, 8801-8809.

(79) Reina, A.; Pradel, C.; Martin, E.; Teuma, E.; Gómez, M. Palladium Nanoparticles Stabilised by Cinchona-based Alkaloids in Glycerol: Efficient Catalysts for Surface Assisted Processes. RSC Adv. 2016, 6, 93205-93216.

(80) Raluy, E.; Grabulosa, A.; Lavedan, P.; Pradel, C.; Muller, G.; Favier, I.; Gómez, M. PStereogenic Phosphines for the Stabilisation of Metal Nanoparticles. A Surface State Study. Catalysts 2016, 6, 213.

(81) Ferri, D.; Bürgi, T. An in Situ Attenuated Total Reflection Infrared Study of a Chiral Catalytic Solid-Liquid Interface: Cinchonidine Adsorption on Pt. J. Am. Chem. Soc. 2001, 123, 12074-12084.

(82) Kraynov, A.; Suchopar, A.; D’Souza, L.; Richards, R. Determination of Geometric Orientation of Adsorbed Cinchonidine on Pt and Fe and Quiphos on Pt Nanoclusters via DRIFTS. Phys. Chem. Chem. Phys. 2006, 8, 1321-1328.

(83) Singh, A. K.; Xu, Q. Synergistic Catalysis over Bimetallic Alloy Nanoparticles. ChemCatChem 2013, 5, 652-676.

(84) Peng, L.; Ringe, E.; Van Duyne, R. P.; Marks, L. D. Segregation in Bimetallic Nanoparticles. Phys. Chem. Chem. Phys. 2015, 17, 27940-27951.

(85) Notar Francesco, I.; Fontaine-Vive, F.; Antoniotti, S. Synergy in the Catalytic Activity of Bimetallic Nanoparticles and New Synthetic Methods for the Preparation of Fine Chemicals. ChemCatChem 2014, 6, 2784-2791.

(86) Massalski, T. B.; Okamoto, H.; Subramanian, P. R.; Kacprzak, L. Binary Alloy Phase Diagrams, 2nd Edition; ASM International: The Materials Information Society: $\mathrm{OH}$, 1989.

(87) Fang, S. S.; Lin, G. W.; Zhang, J. L.; Zhou, Z. Q. The Maximum Solid Solubility of the Transition Metals in Palladium. Int. J. Hydrogen Energy 2002, 27, 329-332.

(88) Dang-Bao, T.; Pla, D.; Favier, I.; Gómez, M. Bimetallic Nanoparticles in Alternative Solvents for Catalytic Purposes. Catalysts 2017, 7, 207.

(89) Silvert, P.-Y.; Vijayakrishnan, V.; Vibert, P.; Herrera-Urbina, R.; Elhsissen, K. Synthesis and Characterization of Nanoscale Ag-Pd Alloy Particles. Nanostruct. Mater. 1996, 7, 611-618. 
(90) Harpeness, R.; Gedanken, A. Microwave Synthesis of Core-Shell Gold/Palladium Bimetallic Nanoparticles. Langmuir 2004, 20, 3431-3434.

(91) Patel, K.; Kapoor, S.; Dave, D. P.; Mukherjee, T. Synthesis of Au, Au/Ag, Au/Pt and $\mathrm{Au} / \mathrm{Pd}$ Nanoparticles Using the Microwave-polyol Method. Res. Chem. Intermed. 2006, 32, 103-113.

(92) Chen, D.; Li, J.; Cui, P.; Liu, H.; Yang, J. Gold-catalyzed Formation of Core-shell Goldpalladium Nanoparticles with Palladium Shells up to Three Atomic Layers. J. Mater. Chem. A 2016, 4, 3813-3821.

(93) Ferrer, D.; Torres-Castro, A.; Gao, X.; Sepúlveda-Guzmán, S.; Ortiz-Méndez, U.; JoséYacamán, M. Three-Layer Core/Shell Structure in Au-Pd Bimetallic Nanoparticles. Nano Lett. 2007, 7, 1701-1705.

(94) Guisbiers, G.; Wautelet, M. Size, Shape and Stress Effects on the Melting Temperature of Nano-polyhedral Grains on a Substrate. Nanotechnol. 2006, 17, 2008-2011.

(95) Mejía-Rosales, S. J.; Fernández-Navarro, C.; Pérez-Tijerina, E.; Blom, D. A.; Allard, L. F.; José-Yacamán, M. On the Structure of Au/Pd Bimetallic Nanoparticles. J. Phys. Chem. C 2007, 111, 1256-1260.

(96) Kim, C.-H.; Lim, J.-S.; Choi, M.-B.; Kim, J.-K.; Yang, H.-S.; Song, S.-J. Polyol Synthesis of Pd/Ag Alloy Nanocrystalline. J. Electrochem. Soc. 2010, 157, E107-E110. Adekoya, J. A.; Dare, E. O.; Mesubi, M. A.; Revaprasadu, N. Polyol Mediated Hexadecylamine Capped Silver Allied Nanobimetallic Particles and Their Fluxional Properties. J. Phys. Chem. Solids 2015, 86, 155-161.

(98) Santoveña, A.; Rodriguez-Proenza, C.; Maya-Cornejo, J. A.; Ruiz-Baltazar, A.; Bahena, D.; Ledesma, J.; Pérez, R.; Esparza, R. Characterization Microstructural and Electrochemical of AgPd Alloy Bimetallic Nanoparticles. MRS Adv. 2017, 2, 2857-2863.

(99) Januszewska, A.; Dercz, G.; Lewera, A.; Jurczakowski, R. Spontaneous Chemical Ordering in Bimetallic Nanoparticles. J. Phys. Chem. C 2015, 119, 19817-19825.

(100) Barcaro, G.; Fortunelli, A.; Polak, M.; Rubinovich, L. Patchy Multishell Segregation in Pd-Pt Alloy Nanoparticles. Nano Lett. 2011, 11, 1766-1769.

(101) Long, N. V.; Asaka, T.; Matsubara, T.; Nogami, M. Shape-controlled Synthesis of Pt-Pd Core-shell Nanoparticles Exhibiting Polyhedral Morphologies by Modified Polyol Method. Acta Mater. 2011, 59, 2901-2907.

(102) Long, N. V.; Ohtaki, M.; Hien, T. D.; Randy, J.; Nogami, M. A Comparative Study of Pt and Pt-Pd Core-shell Nanocatalysts. Electrochim. Acta 2011, 56, 9133-9143.

(103) Nguyen, V. L.; Ohtaki, M.; Matsubara, T.; Cao, M. T.; Nogami, M. New Experimental Evidences of Pt-Pd Bimetallic Nanoparticles with Core-Shell Configuration and Highly Fine-Ordered Nanostructures by High-Resolution Electron Transmission Microscopy. $J$. Phys. Chem. C 2012, 116, 12265-12274.

(104) Esparza, R.; García-Ruiz, A. F.; Salazar, J. V.; Pérez, R.; José-Yacamán, M. Structural Characterization of Pt-Pd Core-shell Nanoparticles by Cs-corrected STEM. J. Nanopart. Res. 2013, 15(1342), 9 pp.

(105) Miyakawa, M.; Hiyoshi, N.; Nishioka, M.; Koda, H.; Sato, K.; Miyazawa, A.; Suzuki, T. M. Continuous Syntheses of Pd@Pt and Cu@Ag Core-shell Nanoparticles Using Microwave-assisted Core Particle Formation Coupled with Galvanic Metal Displacement. Nanoscale 2014, 6, 8720-8725. 
(106) Cong, C.; Nakayama, S.; Maenosono, S.; Harada, M. Microwave-Assisted Polyol Synthesis of Pt/Pd and Pt/Rh Bimetallic Nanoparticles in Polymer Solutions Prepared by Batch and Continuous-Flow Processing. Ind. Eng. Chem. Res. 2018, 57, 179-190.

(107) Wang, J. H.; Wang, K.-W.; Chen, T.-L.; Wang, C.-H.; Huang, Y.-M.; Hsu, Y.-Y.; Chen, T.-Y. Local Heterojunctions of Atomic Pt Clusters Boost the Oxygen Reduction Activity of Rucore@Pdshell Nanocrystallites. J. Mater. Chem. A 2016, 4, 17848-17856.

(108) Kusada, K.; Kobayashi, H.; Ikeda, R.; Kubota, Y.; Takata, M.; Toh, S.; Yamamoto, T.; Matsumura, S.; Sumi, N.; Sato, K.; Nagaoka, K.; Kitagawa, H. Solid Solution Alloy Nanoparticles of Immiscible Pd and Ru Elements Neighboring on Rh: Changeover of the Thermodynamic Behavior for Hydrogen Storage and Enhanced CO-Oxidizing Ability. $J$. Am. Chem. Soc. 2014, 136, 1864-1871.

(109) Kang, X.; Miao, K.; Guo, Z.; Zou, J.; Shi, Z.; Lin, Z.; Huang, J.; Chen, S. PdRu Alloy Nanoparticles of Solid Solution in Atomic Scale: Size Effects on Electronic Structure and Catalytic Activity Towards Electrooxidation of Formic Acid and Methanol. J. Catal. 2018, 364, 183-191.

(110) Miao, K.; Luo, Y.; Zou, J.; Yang, J.; Zhang, F.; Huang, L.; Huang, J.; Kang, X.; Chen, S. $\mathrm{PdRu}$ Alloy Nanoparticles of Solid Solution in Atomic Scale: Outperformance Towards Formic Acid Electro-oxidation in Acidic Medium. Electrochim. Acta 2017, 251, 588-594.

(111) Asano, S.; Maki, T.; Sebastian, V.; Jensen, K. F.; Mae, K. Revealing the Formation Mechanism of Alloyed Pd-Ru Nanoparticles: A Conversion Measurement Approach Utilizing a Microflow Reactor. Langmuir 2019, 35, 2236-2243.

(112) Dawood, F.; Leonard, B. M.; Schaak, R. E. Oxidative Transformation of Intermetallic Nanoparticles: An Alternative Pathway to Metal/Oxide Nanocomposites, Textured Ceramics, and Nanocrystalline Multimetal Oxides. Chem. Mater. 2007, 19, 4545-4550.

(113) Roslova, M.; Opherden, L.; Veremchuk, I.; Spillecke, L.; Kirmse, H.; Herrmannsdörfer, T.; Wosnitza, J.; Doert, T.; Ruck, M. Downscaling Effect on the Superconductivity of Pd3Bi2X2 (X $=$ S or Se) Nanoparticles Prepared by Microwave-Assisted Polyol Synthesis. Inorg. Chem. 2016, 55, 8808-8815.

(114) Sun, S.; Murray, C. B.; Weller, D.; Folks, L.; Moser, A. Monodisperse FePt Nanoparticles and Ferromagnetic FePt Nanocrystal Superlattices. Science 2000, 287, 1989-1992.

(115) Hou, Y.; Kondoh, H.; Kogure, T.; Ohta, T. Preparation and Characterization of Monodisperse FePd Nanoparticles. Chem. Mater. 2004, 16, 5149-5152.

(116) Watanabe, K.; Kura, H.; Sato, T. Transformation to L10 Structure in FePd Nanoparticles Synthesized by Modified Polyol Process. Sci. Tech. Adv. Mater. 2006, 7, 145-149.

(117) Saravanan, P.; Rao, K. S.; Sivaprahasam, D.; Chandrasekaran, V. Consolidation of FePd Nanoparticles by Spark Plasma Sintering. Intermetallics 2010, 18, 2262-2265.

(118) Castellanos-Rubio, I.; Insausti, M.; de Muro, I. G.; Arias-Duque, D. C.; HernándezGarrido, J. C.; Rojo, T.; Lezama, L. The impact of the Chemical Synthesis on the Magnetic Properties of Intermetallic PdFe Nanoparticles. J. Nanopart. Res. 2015, 17(229), 13 pp.

(119) Fleurier, R.; Bhattacharyya, S.; Saboungi, M.-L.; Raimboux, N.; Simon, P.; Kliava, J.; Magrez, A.; Feher, T.; Forro, L.; Salvetat, J.-P. Increase in the Curie Temperature and Magnetic Anisotropy in FePd/Pt-iron Oxide Core-shell Nanoparticles. J. Appl. Phys. 2009, 106, 073903. 
(120) Jain, R.; Gopinath, C. S. New Strategy Toward a Dual Functional Nanocatalyst at Ambient Conditions: Influence of the Pd-Co Interface in the Catalytic Activity of Pd@Co Core-Shell Nanoparticles. ACS Appl. Mater. Interfaces 2018, 10, 41268-41278.

(121) Lu, P.; Teranishi, T.; Asakura, K.; Miyake, M.; Toshima, N. Polymer-protected Ni/Pd Bimetallic Nano-Clusters: Preparation, Characterization and Catalysis for Hydrogenation of Nitrobenzene. J. Phys. Chem. B 1999, 103, 9673-9682.

(122) Ai, F.; Yao, A.; Huang, W.; Wang, D.; Zhang, X. Synthesis of PVP-protected NiPd Nanoalloys by Modified Polyol Process and their Magnetic Properties. Physica E Low Dimens. Syst. Nanostruct. 2010, 42, 1281-1286.

(123) Hokenek, S.; Bennett, C.; Kuhn, J. N. Synthesis of Ni-Pd Nanocubes and Nanorods with High Selectivity Through a Modified Polyol Process. J. Cryst. Growth 2013, 374, 18-22.

(124) Toshima, N.; Wang, Y. Preparation and Catalysis of Novel Colloidal Dispersions of Copper/Noble Metal Bimetallic Clusters. Langmuir 1994, 10, 4574-4580.

(125) Lu, P.; Dong, J.; Toshima, N. Surface-Enhanced Raman Scattering of a Cu/Pd Alloy Colloid Protected by Poly(N-vinyl-2-pyrrolidone). Langmuir 1999, 15, 7980-7992.

(126) Dang-Bao, T.; Pradel, C.; Favier, I.; Gómez, M. Bimetallic Nanocatalysts in Glycerol for Applications in Controlled Synthesis. A Structure-Reactivity Relationship Study. ACS Appl. Nano Mater. 2019, 2, 1033-1044.

(127) Siddiqi, K. S.; Husen, A. Fabrication of Metal Nanoparticles from Fungi and Metal Salts: Scope and Application. Nanoscale Res. Lett. 2016, 11, 98.

(128) Golinska, P.; Wypij, M.; Ingle, A. P.; Gupta, I.; Dahm, H.; Rai, M. Biogenic Synthesis of Metal Nanoparticles from Actinomycetes: Biomedical Applications and Cytotoxicity. Appl. Microbiol. Biotechnol. 2014, 98, 8083-8097.

(129) Alam, M. N.; Roy, N.; Mandal, D.; Begum, N. A. Green Chemistry for Nanochemistry: Exploring Medicinal Plants for the Biogenic Synthesis of Metal NPs with Fine-tuned Properties. RSC Adv. 2013, 3, 11935-11956.

(130) Menon, S.; S, R.; S, V. K. A Review on Biogenic Synthesis of Gold Nanoparticles, Characterization, and its Applications. Res. Eff. Technol. 2017, 3, 516-527.

(131) Siddiqi, K. S.; Husen, A.; Rao, R. A. K. A Review on Biosynthesis of Silver Nanoparticles and their Biocidal Properties. J. Nanobiotechnol. 2018, 16, 14.

(132) Qazi, F.; Hussain, Z.; Tahir, M. N. Advances in Biogenic Synthesis of Palladium Nanoparticles. RSC Adv. 2016, 6, 60277-60286.

(133) Vishnukumar, P.; Vivekanandhan, S.; Muthuramkumar, S. Plant-Mediated Biogenic Synthesis of Palladium Nanoparticles: Recent Trends and Emerging Opportunities. ChemBioEng Rev. 2017, 4, 18-36.

(134) Nadagouda, M. N.; Varma, R. S. Green Synthesis of Silver and Palladium Nanoparticles at Room Temperature Using Coffee and Tea Extract. Green Chem. 2008, 10, 859-862.

(135) Sheny, D. S.; Philip, D.; Mathew, J. Rapid Green Synthesis of Palladium Nanoparticles Using the Dried Leaf of Anacardium Occidentale. Spectroc. Acta A 2012, 91, 35-38.

(136) Yang, X.; Li, Q.; Wang, H.; Huang, J.; Lin, L.; Wang, W.; Sun, D.; Su, Y.; Opiyo, J. B.; Hong, L.; Wang, Y.; He, N.; Jia, L. Green Synthesis of Palladium Nanoparticles Using Broth of Cinnamomum Camphora Leaf. J. Nanopart. Res. 2010, 12, 1589-1598.

(137) Khan, M.; Khan, M.; Kuniyil, M.; Adil, S. F.; Al-Warthan, A.; Alkhathlan, H. Z.; Tremel, W.; Tahir, M. N.; Siddiqui, M. R. H. Biogenic Synthesis of Palladium Nanoparticles Using Pulicaria Glutinosa Extract and their Catalytic Activity Towards the Suzuki Coupling Reaction. Dalton Trans. 2014, 43, 9026-9031. 
(138) Rakibuddin, M; Kim, H. Facile Green Syntheses of Palladium Nanoparticles Using Fruit and Aloe Vera Juices, and their Surface-enhanced Raman Scattering (SERS) Activities. Mater. Res. Exp. 2018, 5, 085001.

(139) Katircioğlu, Z.; Şakalak, H.; Ulaşan, M.; Gören, A. C.; Yavuz, M. S. Facile Synthesis of "Green" Gold Nanocrystals Using Cynarin in an Aqueous Solution. Appl. Surf. Sci. 2014, 318, 191-198.

(140) Kalaiselvi, A.; Roopan, S. M.; Madhumitha, G.; Ramalingam, C.; Elango, G. Synthesis and Characterization of Palladium Nanoparticles Using Catharanthus Roseus Leaf Extract and its Application in the Photo-catalytic Degradation. Spectroc. Acta A 2015, 135, 116-119.

(141) Mohan Kumar, K.; Mandal, B. K.; Siva Kumar, K.; Sreedhara Reddy, P.; Sreedhar, B. Biobased Green Method to Synthesise Palladium and Iron Nanoparticles Using Terminalia Chebula Aqueous Extract. Spectroc. Acta A 2013, 102, 128-133.

(142) Zhan, G.; Huang, J.; Du, M.; Abdul-Rauf, I.; Ma, Y.; Li, Q. Green Synthesis of Au-Pd Bimetallic Nanoparticles: Single-step Bioreduction Method with Plant Extract. Mater. Lett. 2011, 65, 2989-2991.

(143) Rylander, P. N. The Catalytic Hydrogenation in Organic Syntheses; Academic Press, 1979.

(144) Ravanchi, M. T. New Advances in Hydrogenation Processes-Fundamentals and Applications, 2017.

(145) Jackson, S. D.; AlAsseel, A. K. A.; Allgeier, A. M.; Hargreaves, J. S. J.; Kelly, G. J.; Kirkwood, K.; Lok, C. M.; Schauermann, S.; Schmidt, S. R.; Sengupta, S. K. Hydrogenation: Catalysts and Processes; De Gruyter, 2018.

(146) Rahman, A.; Uahengo, V.; Daniel, L. S. Green Chemistry Concept: Applications of Catalysis in Pharmacuetical Industry. Glob Drugs Ther. 2017, 2, 1-6.

(147) Cheddie, D. Ammonia as a Hydrogen Source for Fuel Cells: a Review; In Hydrogen Energy-Challenges and Perspectives; IntechOpen, 2012.

(148) Kumar, A.; Chakraborty, J. P.; Singh, R. Bio-oil: the Future of Hydrogen Generation. Biofuels 2017, 8, 663-674.

(149) Bowker, M. Sustainable Hydrogen Production by the Application of Ambient Temperature Photocatalysis. Green Chem. 2011, 13, 2235-2246.

(150) Wang, D.; Astruc, D. The Golden Age of Transfer Hydrogenation. Chem. Rev. 2015, 115, 6621-6686.

(151) Karem, S.; Catharina, N.; Helmut, S. Green Solvents in Organic Synthesis: An Overview II. Curr. Org. Chem. 2016, 20, 1576-1583.

(152) Chen, X.; Shen, K.; Ding, D.; Chen, J.; Fan, T.; Wu, R.; Li, Y. Solvent-Driven Selectivity Control to Either Anilines or Dicyclohexylamines in Hydrogenation of Nitroarenes over a Bifunctional Pd/MIL-101 Catalyst. ACS Catal. 2018, 8, 10641-10648.

(153) Fajt, V.; Kurc, L.; Červený, L. The Effect of Solvents on the Rate of Catalytic Hydrogenation of 6-Ethyl-1,2,3,4-tetrahydroanthracene-9,10-dione. Int. J. Chem. Kinet. 2008, 40, 240-252.

(154) Takagi, H.; Isoda, T.; Kusakabe, K.; Morooka, S. Effects of Solvents on the Hydrogenation of Mono-Aromatic Compounds Using Noble-Metal Catalysts. Energ. Fuels 1999, 13, 1191-1196.

(155) Giorgianni, G.; Abate, S.; Centi, G.; Perathoner, S.; van Beuzekom, S.; Soo-Tang, S.-H.; Van der Waal, J. C. Effect of the Solvent in Enhancing the Selectivity to Furan 
Derivatives in the Catalytic Hydrogenation of Furfural. ACS Sustainable Chem. Eng. 2018, 6, 16235-16247.

(156) Murzin, D. Y. Solvent Effects in Catalysis: Implementation for Modelling of Kinetics. Catal. Sci. Technol. 2016, 6, 5700-5713.

(157) Brunner, E. Solubility of Hydrogen in Alcohols. Ber. Bunsenges. Phys. Chem. 1979, 83, 715-721.

(158) d'Angelo, J. V. H.; Francesconi, A. Z. Gas-Liquid Solubility of Hydrogen in n-Alcohols $(1 \leqslant \mathrm{n} \leqslant 4)$ at Pressures from 3.6 MPa to $10 \mathrm{MPa}$ and Temperatures from $298.15 \mathrm{~K}$ to 525.15 K. J. Chem. Eng. Data 2001, 46, 671-674.

(159) Battino, R.; Rettich, T. R.; Tominaga, T. The Solubility of Oxygen and Ozone in Liquids. J. Phys. Chem. Ref. Data 1983, 12, 163-178.

(160) de Vries, J. G.; Elsevier, C. J. The Handbook of Homogeneous Hydrogenation; Wiley$\mathrm{VCH}, 2007$.

(161) Narayanan, R. Nanocatalysts for Hydrogenation Reactions; In Nanocatalysis Synthesis and Applications; Polshettiwar, V.;Asefa, T., Eds., 2013.

(162) Yan, N.; Xiao, C.; Kou, Y. Transition Metal Nanoparticle Catalysis in Green Solvents. Coord. Chem. Rev. 2010, 254, 1179-1218.

(163) Guo, M.; Li, H.; Ren, Y.; Ren, X.; Yang, Q.; Li, C. Improving Catalytic Hydrogenation Performance of Pd Nanoparticles by Electronic Modulation Using Phosphine Ligands. ACS Catal. 2018, 8, 6476-6485.

(164) Chen, H.; He, Y.; Pfefferle, L. D.; Pu, W.; Wu, Y.; Qi, S. Phenol Catalytic Hydrogenation over Palladium Nanoparticles Supported on Metal-Organic Frameworks in the Aqueous Phase. Chem CatChem 2018, 10, 2558-2570.

(165) da Silva, F. P.; Fiorio, J. L.; Rossi, L. M. Tuning the Catalytic Activity and Selectivity of Pd Nanoparticles Using Ligand-Modified Supports and Surfaces. ACS Omega 2017, 2, 6014-6022.

(166) Isabelle, F.; David, M.; Emmanuelle, T.; Montserrat, G. Palladium Nanoparticles Applied in Organic Synthesis as Catalytic Precursors. Curr. Org. Chem. 2011, 15, 3127-3174.

(167) Graham, T. XXXVI.-On the Relation of Hydrogen to Palladium. J. Chem. Soc. 1869, 22, 419-432.

(168) Parker, S. F.; Walker, H. C.; Callear, S. K.; Grünewald, E.; Petzold, T.; Wolf, D.; Möbus, K.; Adam, J.; Wieland, S. D.; Jiménez-Ruiz, M.; Albers, P. W. The Effect of Particle Size, Morphology and Support on the Formation of Palladium Hydride in Commercial Catalysts. Chem. Sci. 2019, 10, 480-489.

(169) Armbrüster, M.; Behrens, M.; Cinquini, F.; Föttinger, K.; Grin, Y.; Haghofer, A.; Klötzer, B.; Knop-Gericke, A.; Lorenz, H.; Ota, A.; Penner, S.; Prinz, J.; Rameshan, C.; Révay, Z.; Rosenthal, D.; Rupprechter, G.; Sautet, P.; Schlögl, R.; Shao, L.; Szentmiklósi, L.; Teschner, D.; Torres, D.; Wagner, R.; Widmer, R.; Wowsnick, G. How to Control the Selectivity of Palladium-based Catalysts in Hydrogenation Reactions: The Role of Subsurface Chemistry. Chem CatChem 2012, 4, 1048-1063.

(170) Yabe, Y.; Sawama, Y.; Monguchi, Y.; Sajiki, H. New Aspect of Chemoselective Hydrogenation Utilizing Heterogeneous Palladium Catalysts Supported by Nitrogen- and Oxygen-containing Macromolecules. Catal. Sci. Technol. 2014, 4, 260-271.

(171) Zhou, H.-F.; Fan, Q.-H.; Tang, W.-J.; Xu, L.-J.; He, Y.-M.; Deng, G.-J.; Zhao, L.-W.; Gu, L.-Q.; Chan, A. S. C. Polyethylene Glycol as an Environmentally Friendly and 
Recyclable Reaction Medium for Enantioselective Hydrogenation. Adv. Synth. Catal. 2006, 348, 2172-2182.

(172) Totten, G. E.; Clinton, N. A. Poly(ethylene Glycol) and Derivatives as Phase Transfer Catalysts. J. Macromol. Sci. C 1998, 38, 77-142.

(173) Kann, N. Recent Applications of Polymer Supported Organometallic Catalysts in Organic Synthesis. Molecules 2010, 15, 6306-6331.

(174) Chen, J.; Spear, S. K.; Huddleston, J. G.; Rogers, R. D. Polyethylene Glycol and Solutions of Polyethylene Glycol as Green Reaction Media. Green Chem. 2005, 7, 64-82.

(175) Carlos Kleber, Z. A.; Luana, M. A. Environmentally Benign Solvents in Organic Synthesis: Current Topics. Curr. Org. Chem. 2005, 9, 195-218.

(176) Chandrasekhar, S.; Prakash, S. J.; Rao, C. L. Poly(ethylene Glycol) (400) as Superior Solvent Medium Against Ionic Liquids for Catalytic Hydrogenations with PtO2. J. Org. Chem. 2006, 71, 2196-2199.

(177) Luo, C.; Zhang, Y.; Wang, Y. Palladium Nanoparticles in Poly(ethylene glycol): an Efficient and Recyclable Catalyst for the Heck Reaction. J. Mol. Catal. A: Chem. 2005, 229, 7-12.

(178) Ma, X.; Jiang, T.; Han, B.; Zhang, J.; Miao, S.; Ding, K.; An, G.; Xie, Y.; Zhou, Y.; Zhu, A. Palladium Nanoparticles in Polyethylene Glycols: Efficient and Recyclable Catalyst System for Hydrogenation of Olefins. Catal. Commun. 2007, 9, 70-74.

(179) Liu, W.; Xu, L.; Lu, G.; Zhang, H. Selective Partial Hydrogenation of Methyl Linoleate Using Highly Active Palladium Nanoparticles in Polyethylene Glycol. ACS Sustainable Chem. Eng. 2017, 5, 1368-1375.

(180) Liu, W.; Lu, G. Partial Hydrogenation of Sunflower Oil-derived FAMEs Catalyzed by the Efficient and Recyclable Palladium Nanoparticles in Polyethylene Glycol. J. Oleo Sci. 2017, 66, 1161-1168.

(181) Huang, T. S.; Wang, Y. H.; Jiang, J. Y.; Jin, Z. L. PEG-stabilized Palladium Nanoparticles: an Efficient and Recyclable Catalyst for the Selective Hydrogenation of 1,5-Cyclooctadiene in Thermoregulated PEG Biphase System. Chin. Chem. Lett. 2008, 19, 102-104.

(182) Niu, M.; Wang, Y.; Li, W.; Jiang, J.; Jin, Z. Thermoregulated Poly(ethylene glycol) Biphasic System with Pd Nanoparticle Catalysts for Selective Hydrogenation of Cinnamaldehyde. Chin. J. Catal. 2013, 34, 674-678.

(183) Pillai, U. R.; Sahle-Demessie, E. Phenanthroline-stabilized Palladium Nanoparticles in Polyethylene Glycol-an Active and Recyclable Catalyst System for the Selective Hydrogenation of Olefins Using Molecular Hydrogen. J. Mol. Catal. A: Chem. 2004, $222,153-158$.

(184) Huang, J.; Jiang, T.; Han, B.; Gao, H.; Chang, Y.; Zhao, G.; Wu, W. Hydrogenation of Olefins Using Ligand-stabilized Palladium Nanoparticles in an Ionic Liquid. Chem. Commun. 2003, 1654-1655.

(185) Alba, E. D.-Á.; Javier, F.; Pascale, C.; Victorio, C. Recent Advances in the Use of Glycerol as Green Solvent for Synthetic Organic Chemistry. Curr. Green Chem. 2014, 1, 51-65.

(186) Tagliapietra, S.; Orio, L.; Palmisano, G.; Penoni, A.; Cravotto, G. Catalysis in Glycerol: a Survey of Recent Advances. Chem. Pap. 2015, 69, 1519. 
(187) Chahdoura, F.; Mallet-Ladeira, S.; Gómez, M. Palladium Nanoparticles in Glycerol: a Clear-cut Catalyst for One-pot Multi-step Processes Applied in the Synthesis of Heterocyclic Compounds. Org. Chem. Front. 2015, 2, 312-318.

(188) Garg, G.; Foltran, S.; Favier, I.; Pla, D.; Medina-González, Y.; Gómez, M. Palladium Nanoparticles Stabilized by Novel Choline-based Ionic Liquids in Glycerol Applied in Hydrogenation Reactions. Catal. Today 2019, doi:10.1016/j.cattod.2019.01.052.

(189) Hidefumi, H.; Yukimichi, N.; Naoki, T.; Koichi, A. Colloidal Rhodium in Polyvinyl Alcohol as Hydrogenation Catalyst of Olefins. Chem. Lett. 1976, 5, 905-910.

(190) Wu, J.; Li, P.; Pan, Y.-T.; Warren, S.; Yin, X.; Yang, H. Surface Lattice-engineered Bimetallic Nanoparticles and their Catalytic Properties. Chem. Soc. Rev. 2012, 41, 80668074.

(191) Gu, J.; Zhang, Y.-W.; Tao, F. Shape Control of Bimetallic Nanocatalysts Through Welldesigned Colloidal Chemistry Approaches. Chem. Soc. Rev. 2012, 41, 8050-8065.

(192) Goodson, F. E.; Cichowicz, M. B. Organic Synthesis Using Metal-Mediated Coupling Reactions; In Encyclopedia of Inorganic and Bioinorganic Chemistry.

(193) de Vries, J. G. Palladium-Catalysed Coupling Reactions; In Organometallics as Catalysts in the Fine Chemical Industry; Beller, M.;Blaser, H.-U., Eds.; Springer Berlin Heidelberg: Berlin, Heidelberg, 2012, pp 1-34.

(194) McGlacken, G. P.; Fairlamb, I. J. S. Palladium-Catalysed Cross-Coupling and Related Processes: Some Interesting Observations That Have Been Exploited in Synthetic Chemistry. Eur. J. Org. Chem. 2009, 2009, 4011-4029.

(195) Ananikov, V. P.; Beletskaya, I. P. Toward the Ideal Catalyst: From Atomic Centers to a "Cocktail” of Catalysts. Organometallics 2012, 31, 1595-1604.

(196) Xie, H.; Fan, T.; Lei, Q.; Fang, W. New Progress in Theoretical Studies on Palladiumcatalyzed C-C Bond-forming Reaction Mechanisms. Sci. China Chem. 2016, 59, 14321447.

(197) Manuel, M.; César, J.-S.; José Rafael, R. Recent Advances in the Heterogeneous Palladium-Catalysed Suzuki Cross-Coupling Reaction. Curr. Org. Chem. 2012, 16, 11281150.

(198) Biffis, A.; Centomo, P.; Del Zotto, A.; Zecca, M. Pd Metal Catalysts for Cross-Couplings and Related Reactions in the 21st Century: A Critical Review. Chem. Rev. 2018, 118, 2249-2295.

(199) Beletskaya Irina, P.; Averin Alexei, D. New Trends in the Cross-coupling and Other Catalytic Reactions. Pure Appl. Chem. 2017, 89, 1413.

(200) Magne, O. S. Use of Nanoparticles as Catalysts in Organic Synthesis - Cross-coupling Reactions. Curr. Org. Chem. 2014, 18, 312-326.

(201) Tsuji, Y.; Fujihara, T. New Design in Homogeneous Palladium Catalysis: Study of Transformation of Group 14 Element Compounds and Development of Nanosize Palladium Catalysts. Bull. Korean Chem. Soc. 2007, 28, 1902-1909.

(202) Jana, R.; Pathak, T. P.; Sigman, M. S. Advances in Transition Metal (Pd,Ni,Fe)Catalyzed Cross-Coupling Reactions Using Alkyl-organometallics as Reaction Partners. Chem. Rev. 2011, 111, 1417-1492.

(203) Moragas, T.; Correa, A.; Martin, R. Metal-Catalyzed Reductive Coupling Reactions of Organic Halides with Carbonyl-Type Compounds. Chem. Eur. J. 2014, 20, 8242-8258. 
(204) Shi, W.; Liu, C.; Lei, A. Transition-metal Catalyzed Oxidative Cross-coupling Reactions to Form $\mathrm{C}-\mathrm{C}$ Bonds Involving Organometallic Reagents as Nucleophiles. Chem. Soc. Rev. 2011, 40, 2761-2776.

(205) Beletskaya Irina, P. Palladium Catalyzed C-C and C-heteroatom Bond Formation Reactions. Pure App. Chem. 1997, 69, 471.

(206) Reetz, M. T.; Westermann, E. Phosphane-Free Palladium-Catalyzed Coupling Reactions: The Decisive Role of Pd Nanoparticles. Angew. Chem., Int. Ed. 2000, 39, 165-168.

(207) de Vries, A. H. M.; Mulders, J. M. C. A.; Mommers, J. H. M.; Henderickx, H. J. W.; de Vries, J. G. Homeopathic Ligand-Free Palladium as a Catalyst in the Heck Reaction. A Comparison with a Palladacycle. Org. Lett. 2003, 5, 3285-3288.

(208) Köhler, K.; Heidenreich, R. G.; Krauter, J. G. E.; Pietsch, J. Highly Active Palladium/Activated Carbon Catalysts for Heck Reactions: Correlation of Activity, Catalyst Properties, and Pd Leaching. Chem. Eur. J. 2002, 8, 622-631.

(209) Beletskaya, I. P.; Cheprakov, A. V. The Heck Reaction as a Sharpening Stone of Palladium Catalysis. Chem. Rev. 2000, 100, 3009-3066.

(210) Deraedt, C.; Astruc, D. "Homeopathic" Palladium Nanoparticle Catalysis of Cross Carbon-Carbon Coupling Reactions. Acc. Chem. Res. 2014, 47, 494-503.

(211) Shaughnessy, K. H. Ch 14 Greener Approaches to Cross-Coupling; In New Trends in Cross-Coupling: Theory and Applications; The Royal Society of Chemistry, 2015, pp 645-696.

(212) Fihri, A.; Bouhrara, M.; Nekoueishahraki, B.; Basset, J.-M.; Polshettiwar, V. Nanocatalysts for Suzuki Cross-coupling Reactions. Chem. Soc. Rev. 2011, 40, 51815203.

(213) Mpungose, P.; Vundla, Z.; Maguire, G.; Friedrich, H. The Current Status of Heterogeneous Palladium Catalysed Heck and Suzuki Cross-Coupling Reactions. Molecules 2018, 23, 1676.

(214) Santoro, S.; Ferlin, F.; Luciani, L.; Ackermann, L.; Vaccaro, L. Biomass-derived Solvents as Effective Media for Cross-coupling Reactions and $\mathrm{C}-\mathrm{H}$ Functionalization Processes. Green Chem. 2017, 19, 1601-1612.

(215) Astruc, D. Palladium Catalysis Using Dendrimers: Molecular Catalysts versus Nanoparticles. Tetrahedron Asymmetry 2010, 21, 1041-1054.

(216) Schwartz, J. Alkane Activation by Oxide-bound Organorhodium Complexes. Acc. Chem. Res. 1985, 18, 302-308.

(217) Gómez, M.; Favier, I. Metal Nanoparticles Dispersed in Solution: Tests to Identify the Catalyst Nature; In Metal Nanoclusters in Catalysis and Materials Science: The Issue of Size Control; Corain, B.;Schmid, G.;Toshima, N., Eds.; Elsevier Science: Amsterdam, 2008, Ch. 31, pp 427-436.

(218) Widegren, J. A.; Finke, R. G. A Review of the Problem of Distinguishing True Homogeneous Catalysis from Soluble or Other Metal-particle Heterogeneous Catalysis under Reducing Conditions. J. Mol. Catal. A Chem. 2003, 198, 317-341.

(219) Corma, A.; Garcia, H.; Leyva, A. Polyethylene Glycol as Scaffold and Solvent for Reusable C-C Coupling Homogeneous Pd Catalysts. J. Catal. 2006, 240, 87-99.

(220) Colacino, E.; Martinez, J.; Lamaty, F.; Patrikeeva, L. S.; Khemchyan, L. L.; Ananikov, V. P.; Beletskaya, I. P. PEG as an Alternative Reaction Medium in Metal-mediated Transformations. Coord. Chem. Rev. 2012, 256, 2893-2920. 
(221) Molnár, Á.; Papp, A. The Use of Polysaccharides and Derivatives in Palladium-catalyzed Coupling Reactions. Catal. Sci. Technol. 2014, 4, 295-310.

(222) Garrett, C. E.; Prasad, K. The Art of Meeting Palladium Specifications in Active Pharmaceutical Ingredients Produced by Pd-Catalyzed Reactions. Adv. Synth. Catal. 2004, 346, 889-900.

(223) Yang, C.; Nolan, S. P. Regio- and Stereoselective Dimerization of Terminal Alkynes to Enynes Catalyzed by a Palladium/Imidazolium System. J. Org. Chem. 2002, 67, 591-593.

(224) Firouzabadi, H.; Iranpoor, N.; Ghaderi, A. Gelatin as a Bioorganic Reductant, Ligand and Support for Palladium Nanoparticles. Application as a Catalyst for Ligand- and Aminefree Sonogashira-Hagihara Reaction. Org. Biomol. Chem. 2011, 9, 865-871.

(225) Sonogashira, K. Development of Pd-Cu Catalyzed Cross-coupling of Terminal Acetylenes with sp2-Carbon Halides. J. Organomet. Chem. 2002, 653, 46-49.

(226) Gazvoda, M.; Virant, M.; Pinter, B.; Košmrlj, J. Mechanism of Copper-free Sonogashira Reaction Operates Through Palladium-palladium Transmetallation. Nat. Commun. 2018, 9, 4814.

(227) Firouzabadi, H.; Iranpoor, N.; Kazemi, F.; Gholinejad, M. Palladium Nano-particles Supported on Agarose as Efficient Catalyst and Bioorganic Ligand for C-C Bond Formation via Solventless Mizoroki-Heck Reaction and Sonogashira-Hagihara Reaction in Polyethylene Glycol (PEG 400). J. Mol. Catal. A: Chem. 2012, 357, 154-161.

(228) Dabiri, M.; Vajargahy, M. P. PdCo Bimetallic Nanoparticles Supported on Threedimensional Graphene as a Highly Active Catalyst for Sonogashira Cross-coupling Reaction. Appl. Organomet. Chem. 2017, 31, e3594 (5 pp).

(229) Hatanaka, Y.; Hiyama, T. Cross-coupling of Organosilanes with Organic Halides Mediated by a Palladium Catalyst and Tris(diethylamino)sulfonium Difluorotrimethylsilicate. J. Org. Chem. 1988, 53, 918-920.

(230) Denmark, S. E.; Werner, N. S. Cross-Coupling of Aromatic Bromides with Allylic Silanolate Salts. J. Am. Chem. Soc. 2008, 130, 16382-16393.

(231) Denmark, S. E.; Smith, R. C.; Chang, W.-T. T.; Muhuhi, J. M. Cross-Coupling Reactions of Aromatic and Heteroaromatic Silanolates with Aromatic and Heteroaromatic Halides. J. Am. Chem. Soc. 2009, 131, 3104-3118.

(232) Denmark, S. E.; Regens, C. S. Palladium-Catalyzed Cross-Coupling Reactions of Organosilanols and Their Salts: Practical Alternatives to Boron- and Tin-Based Methods. Acc. Chem. Res. 2008, 41, 1486-1499.

(233) Alacid, E.; Nájera, C. Aqueous Sodium Hydroxide Promoted Cross-Coupling Reactions of Alkenyltrialkoxysilanes under Ligand-Free Conditions. J. Org. Chem. 2008, 73, $2315-$ 2322.

(234) Srimani, D.; Sawoo, S.; Sarkar, A. Convenient Synthesis of Palladium Nanoparticles and Catalysis of Hiyama Coupling Reaction in Water. Org. Lett. 2007, 9, 3639-3642.

(235) Reina, A.; Serrano-Maldonado, A.; Teuma, E.; Martin, E.; Gómez, M. Palladium Nanocatalysts in Glycerol: Tuning the Reactivity by Effect of the Stabilizer. Catal. Commun. 2018, 104, 22-27.

(236) Srimani, D.; Bej, A.; Sarkar, A. Palladium Nanoparticle Catalyzed Hiyama Coupling Reaction of Benzyl Halides. J. Org. Chem. 2010, 75, 4296-4299.

(237) Heck, R. F.; Nolley, J. P. Palladium-catalyzed Vinylic Hydrogen Substitution Reactions with Aryl, Benzyl, and Styryl Halides. J. Org. Chem. 1972, 37, 2320-2322. 
(238) Tsutomu, M.; Kunio, M.; Atsumu, O. Arylation of Olefin with Aryl Iodide Catalyzed by Palladium. Bull. Chem. Soc. Jpn. 1971, 44, 581-581.

(239) Rauf, W.; Brown, J. M. Reactive Intermediates in Catalytic Alkenylation; Pathways for Mizoroki-Heck, Oxidative Heck and Fujiwara-Moritani Reactions. Chem. Commun. 2013, 49, 8430-8440.

(240) Chen, Y.; Frey, H.; Thomann, R.; Stiriba, S.-E. Optically Active Amphiphilic Hyperbranched Polyglycerols as Templates for Palladium Nanoparticles. Inorg. Chim. Acta 2006, 359, 1837-1844.

(241) Jumde, R. P.; Marelli, M.; Scotti, N.; Mandoli, A.; Psaro, R.; Evangelisti, C. Ultrafine Palladium Nanoparticles Immobilized into Poly(4-vinylpyridine)-based Porous Monolith for Continuous-flow Mizoroki-Heck Reaction. J. Mol. Catal. A: Chem. 2016, 414, 55-61.

(242) Tabatabaei Rezaei, S. J.; Shamseddin, A.; Ramazani, A.; Mashhadi Malekzadeh, A.; Azimzadeh Asiabi, P. Palladium Nanoparticles Immobilized on Amphiphilic and Hyperbranched Polymer-functionalized Magnetic Nanoparticles: An Efficient Semiheterogeneous Catalyst for Heck Reaction. Appl. Organomet. Chem. 2017, 31, e3707 (10 pp).

(243) Miyaura, N.; Yamada, K.; Suzuki, A. A New Stereospecific Cross-coupling by the Palladium-catalyzed Reaction of 1-Alkenylboranes with 1-Alkenyl or 1-Alkynyl Halides. Tetrahedron Lett. 1979, 20, 3437-3440.

(244) Razler, T. M.; Hsiao, Y.; Qian, F.; Fu, R.; Khan, R. K.; Doubleday, W. A Preparatively Convenient Ligand-Free Catalytic PEG 2000 Suzuki-Miyaura Coupling. J. Org. Chem. 2009, 74, 1381-1384.

(245) Paul, S.; Islam, M. M.; Islam, S. M. Suzuki-Miyaura Reaction by Heterogeneously Supported Pd in Water: Recent Studies. RSC Adv. 2015, 5, 42193-42221.

(246) Bej, A.; Srimani, D.; Sarkar, A. Palladium Nanoparticle Catalysis: Borylation of Aryl and Benzyl Halides and One-pot Biaryl Synthesis via Sequential Borylation-Suzuki-Miyaura Coupling. Green Chem. 2012, 14, 661-667.

(247) Han, W.; Liu, C.; Jin, Z. Aerobic Ligand-free Suzuki Coupling Reaction of Aryl Chlorides Catalyzed by in situ Generated Palladium Nanoparticles at Room Temperature. Adv. Synth. Catal. 2008, 350, 501-508.

(248) Moreno-Mañas, M.; Pérez, M.; Pleixats, R. Palladium-Catalyzed Suzuki-Type SelfCoupling of Arylboronic Acids. A Mechanistic Study. J. Org. Chem. 1996, 61, 23462351.

(249) Adamo, C.; Amatore, C.; Ciofini, I.; Jutand, A.; Lakmini, H. Mechanism of the Palladium-Catalyzed Homocoupling of Arylboronic Acids: Key Involvement of a Palladium Peroxo Complex. J. Am. Chem. Soc. 2006, 128, 6829-6836.

(250) Thomas, A. A.; Denmark, S. E. Pre-transmetalation Intermediates in the Suzuki-Miyaura Reaction Revealed: the Missing Link. Science 2016, 352, 329-332.

(251) Thomas, A. A.; Zahrt, A. F.; Delaney, C. P.; Denmark, S. E. Elucidating the Role of the Boronic Esters in the Suzuki-Miyaura Reaction: Structural, Kinetic, and Computational Investigations. J. Am. Chem. Soc. 2018, 140, 4401-4416.

(252) Ibragimova, A. I.; Vagapova, G. I.; Gainanova, G. A.; Syakaev, V. V.; Zakharov, V. M.; Rizvanov, I. K.; Zakharova, L. Y.; Latypov, S. K.; Konovalov, A. I. The Polyethylene Glycol-sodium Dodecylsulfate-PdC12-water Supramolecular Catalytic System for the Suzuki Reaction. Mendeleev Commun. 2011, 21, 53-55. 
(253) Cho, J. K.; Najman, R.; Dean, T. W.; Ichihara, O.; Muller, C.; Bradley, M. Captured and Cross-Linked Palladium Nanoparticles. J. Am. Chem. Soc. 2006, 128, 6276-6277.

(254) Gholinejad, M.; Zareh, F.; Najera, C. Nitro Group Reduction and Suzuki Reaction Catalysed by Palladium Supported on Magnetic Nanoparticles Modified with Carbon Quantum Dots Generated from Glycerol and Urea. Appl. Organomet. Chem. 2018, 32, e3984 (5 pp).

(255) Mallikarjuna, K.; Bathula, C.; Buruga, K.; Shrestha, N. K.; Noh, Y.-Y.; Kim, H. Green Synthesis of Palladium Nanoparticles using Fenugreek Tea and their Catalytic Applications in Organic Reactions. Mater. Lett. 2017, 205, 138-141.

(256) Moumen, A.; Halim, W.; Jaffal, S.; Abderrafi, K.; Eddahbi, A.; Sebti, S.; Ouaskit, S. Microwave Assisted Synthesis of Palladium Nanoparticles in an Aqueous Emulsion of Copolymer: Application to Catalysis. J. Clust. Sci. 2017, 28, 2817-2832.

(257) Li, B.; Yu, Y.; Zhao, P.; Zhang, S. Triazole-Containing Dendrimer-like Core CrossLinked Micelles that Stabilize Pd Nanoparticles as Heterogenized Homogeneous Catalysts for Room-Temperature Suzuki-Miyaura Reactions in Water. Chem. - Asian J. 2016, 11, 3550-3556.

(258) Doherty, S.; Knight, J. G.; Backhouse, T.; Abood, E.; Al-shaikh, H.; Clemmet, A. R.; Ellison, J. R.; Bourne, R. A.; Chamberlain, T. W.; Stones, R.; Warren, N. J.; Fairlamb, I. A. J.; Lovelock, K. R. J. Heteroatom Donor-Decorated Polymer-Immobilized Ionic Liquid Stabilized Palladium Nanoparticles: Efficient Catalysts for Room-Temperature Suzuki-Miyaura Cross-Coupling in Aqueous Media. Adv. Synth. Catal. 2018, 360, 37163731.

(259) Garcia-Suarez, E. J.; Lara, P.; Garcia, A. B.; Ojeda, M.; Luque, R.; Philippot, K. Efficient and Recyclable Carbon-supported Pd Nanocatalysts for the Suzuki-Miyaura Reaction in Aqueous-based Media: Microwave vs. Conventional Heating. Appl. Catal., A 2013, 468, 59-67.

(260) Nikoorazm, M.; Ghorbani-Choghamarani, A.; Ghobadi, M.; Massahi, S. PdSBT@MCM-41: As an Efficient, Stable and Recyclable Organometallic Catalyst for C-C Coupling Reactions and Synthesis of 5-Substituted Tetrazoles. Appl. Organomet. Chem. 2017, 31, e3848 (9 pp).

(261) Naghipour, A.; Fakhri, A. Heterogeneous Fe3O4@chitosan-Schiff Base Pd Nanocatalyst: Fabrication, Characterization and Application as Highly Efficient and Magneticallyrecoverable Catalyst for Suzuki-Miyaura and Heck-Mizoroki C-C Coupling Reactions. Catal. Commun. 2016, 73, 39-45.

(262) Wang, J.; Song, G.; Peng, Y. Reusable Pd Nanoparticles Immobilized on Functional Ionic Liquid Co-polymerized with Styrene for Suzuki Reactions in Water-ethanol Solution. Tetrahedron Lett. 2011, 52, 1477-1480.

(263) Ishiyama, T.; Kizaki, H.; Hayashi, T.; Suzuki, A.; Miyaura, N. Palladium-Catalyzed Carbonylative Cross-Coupling Reaction of Arylboronic Acids with Aryl Electrophiles: Synthesis of Biaryl Ketones. J. Org. Chem. 1998, 63, 4726-4731.

(264) Zhou, Q.; Wei, S.; Han, W. In Situ Generation of Palladium Nanoparticles: Ligand-Free Palladium Catalyzed Pivalic Acid Assisted Carbonylative Suzuki Reactions at Ambient Conditions. J. Org. Chem. 2014, 79, 1454-1460.

(265) Santra, S.; Ranjan, P.; Mandal, S. K.; Ghorai, P. K. Living Nanocatalyst for Effective Synthesis of Symmetrical Biaryls. Inorg. Chim. Acta 2011, 372, 47-52. 
(266) Favier, I.; Toro, M.-L.; Lecante, P.; Pla, D.; Gómez, M. Palladium-mediated Radical Homocoupling Reactions: a Surface Catalytic Insight. Catal. Sci. Technol. 2018, 8, 47664773.

(267) Staszek, M.; Siegel, J.; Rimpelová, S.; Lyutakov, O.; Švorčík, V. Cytotoxicity of Noble Metal Nanoparticles Sputtered into Glycerol. Mater. Lett. 2015, 158, 351-354. 
TOC

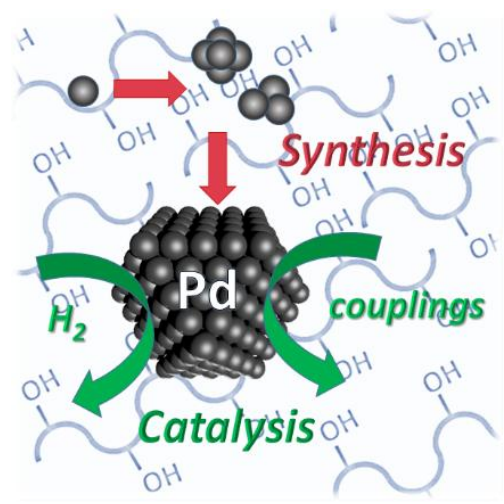

